Louisiana State University

LSU Digital Commons

Faculty Publications

Department of Physics \& Astronomy

$12-2021$

\title{
Diving Beneath the Sea of Stellar Activity: Chromatic Radial Velocities of the Young AU Mic Planetary System
}

\author{
Bryson Cale \\ George Mason Univ, 4400 Univ Dr, Fairfax, VA 22030 USA, bryson.cale1@gmail.com \\ Michael Reefe \\ George Mason Univ, 4400 Univ Dr, Fairfax, VA 22030 USA \\ Peter Plavchan \\ George Mason Univ, 4400 Univ Dr, Fairfax, VA 22030 USA \\ Angelle Tanner \\ Mississippi State Univ, 75 BS Hood Rd, Mississippi State, MS 39762 USA \\ Eric Gaidos \\ Univ Hawaii Manoa, 1680 East West Rd, Honolulu, HI 96822 USA \\ Follow this and additional works at: https://digitalcommons.Isu.edu/physics_astronomy_pubs

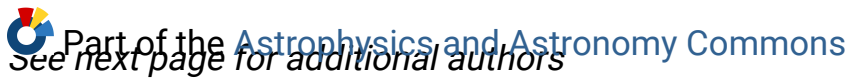

\section{Recommended Citation}

Cale, B., Reefe, M., Plavchan, P., Tanner, A., Gaidos, E., Gagne, J., Gao, P., Kane, S. R., Bejar, V. J., Lodieu, N., Anglada-Escude, G., Ribas, I., Palle, E., Quirrenbach, A., Amado, P., Reiners, A., Caballero, J. A., Osorio, M., Dreizler, S., Howard, A. W., Fulton, B. J., Wang, S., Collins, K. I., El Mufti, M., Wittrock, J., Gilbert, E. A., Barclay, T., Klein, B., Martioli, E., Wittenmyer, R., Wright, D., Addison, B., Hirano, T., Tamura, M., Kotani, T., Narita, N., Vermilion, D., Lee, R. A., Geneser, C., Teske, J., Quinn, S. N., Latham, D. W., Esquerdo, G. A., Calkins, M. L., Berlind, P., Zohrabi, F., Stibbards, C., Kotnana, S., Jenkins, J., Twicken, J. D., Henze, C., Kidwell, Jr., R., Burke, C., Villasenor, J., \& Boyd, P. (2021). Diving Beneath the Sea of Stellar Activity: Chromatic Radial Velocities of the Young AU Mic Planetary System. ASTRONOMICAL JOURNAL, 162 (6) https://doi.org/10.3847/1538-3881/ac2c80

This Article is brought to you for free and open access by the Department of Physics \& Astronomy at LSU Digital Commons. It has been accepted for inclusion in Faculty Publications by an authorized administrator of LSU Digital Commons. For more information, please contact ir@lsu.edu. 


\section{Authors}

Bryson Cale; Michael Reefe; Peter Plavchan; Angelle Tanner; Eric Gaidos; Jonathan Gagne; Peter Gao; Stephen R. Kane; Victor J. S. Bejar; Nicolas Lodieu; Guillem Anglada-Escude; Ignasi Ribas; Enric Palle; Andreas Quirrenbach; Pedro Amado; Ansgar Reiners; Jose A. Caballero; Maria Rosa Zapatero Osorio; Stefan Dreizler; Andrew W. Howard; Benjamin J. Fulton; Sharon Xuesong Wang; Kevin I. Collins; Mohammed El Mufti; Justin Wittrock; Emily A. Gilbert; Thomas Barclay; Baptiste Klein; Eder Martioli; Robert Wittenmyer; Duncan Wright; Brett Addison; Teruyuki Hirano; Motohide Tamura; Takayuki Kotani; Norio Narita; David Vermilion; Rena A. Lee; Claire Geneser; Johanna Teske; Samuel N. Quinn; David W. Latham; Gilbert A. Esquerdo; Michael L. Calkins; Perry Berlind; Farzaneh Zohrabi; Caitlin Stibbards; Srihan Kotnana; Jon Jenkins; Joseph D. Twicken; Christopher Henze; Richard Kidwell, Jr.; Christopher Burke; Joel Villasenor; and Patricia Boyd 


\title{
Diving Beneath the Sea of Stellar Activity: Chromatic Radial Velocities of the Young AU Mic Planetary System
}

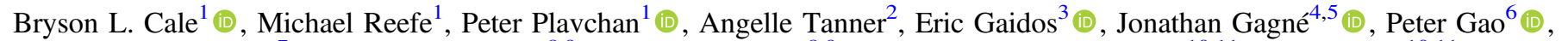
Stephen R. Kane ${ }^{7}$ (i), Víctor J. S. Béjar ${ }^{8,9}$ (i), Nicolas Lodieu ${ }^{8,9}$ (i) , Guillem Anglada-Escudé ${ }^{10,11}$, Ignasi Ribas ${ }^{10,11}$ (1)

Enric Palle ${ }^{8,9}(\mathbb{1})$, Andreas Quirrenbach ${ }^{12}$, Pedro J. Amado ${ }^{13}$, Ansgar Reiners ${ }^{14}$, José A. Caballero ${ }^{15}$, María Rosa Zapatero Osorio ${ }^{16}$, Stefan Dreizler ${ }^{14}$, Andrew W. Howard ${ }^{17}$ (D), Benjamin J. Fulton ${ }^{18}$ (1), Sharon Xuesong Wang ${ }^{19}$, Kevin I. Collins ${ }^{1}$ (D),

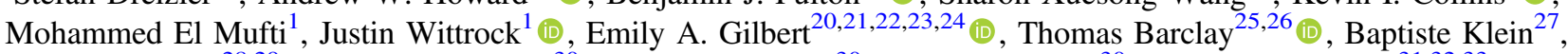
Eder Martioli 28,29 , Robert Wittenmyer ${ }^{30}$ (1) , Duncan Wright $^{30}$ (1) , Brett Addison $^{30}$ (1) , Teruyuki Hirano $^{31,32,33}$ (1), Motohide Tamura ${ }^{31,32,34}$ (1), Takayuki Kotani ${ }^{31,32,33}$, Norio Narita ${ }^{31,35,36,37}$ (1), David Vermilion ${ }^{1}$, Rena A. Lee ${ }^{38}$, Claire Geneser ${ }^{2}$, Johanna Teske $^{39}$, Samuel N. Quinn ${ }^{40}$ (1), David W. Latham ${ }^{40}$ (i) , Gilbert A. Esquerdo $^{40}$ (1) , Michael L. Calkins $^{40}$ (1) , Perry Berlind $^{40}$, Farzaneh Zohrabi $^{41}$, Caitlin Stibbards ${ }^{1}$, Srihan Kotnana ${ }^{1,42}$, Jon Jenkins ${ }^{43}$ (1), Joseph D. Twicken ${ }^{43,44}$ (1) , Christopher Henze $^{43}$, Richard Kidwell, Jr. ${ }^{45}$, Christopher Burke ${ }^{46}$ (1) , Joel Villaseñor $^{46}$, and Patricia Boyd ${ }^{47}$ (1)

${ }^{1}$ George Mason University, 4400 University Drive, Fairfax, VA 22030, USA; bryson.cale1@ gmail.com

${ }_{2}^{2}$ Mississippi State University, 75 B. S. Hood Road, Mississippi State, MS 39762, USA

${ }^{3}$ University of Hawai'i at Manoa, 1680 East-West Road, Honolulu, HI 96822, USA

${ }^{4}$ Planétarium Rio Tinto Alcan, Espace pour la vie, 4801 av. Pierre-De Coubertin, Montréal, QC H1V 3V4, Canada

${ }^{5}$ Institute for Research on Exoplanets, Université de Montréal, Département de Physique, C.P. 6128 Succ. Centre-ville, Montréal, QC H3C 3J7, Canada

${ }^{6}$ University of California, Santa Cruz, 1156 High Street, Santa Cruz, CA 95064, USA

${ }^{7}$ University of California, Riverside, 900 University Avenue Riverside, CA 92521, USA

${ }^{8}$ Instituto de Astrofísica de Canarias, E-38205 La Laguna, Tenerife, Spain

${ }^{9}$ Departamento de Astrofísica, Universidad de La Laguna, E-38206 La Laguna, Tenerife, Spain

${ }^{10}$ Institut de Ciències de l'Espai (ICE, CSIC), Campus UAB, Can Magrans s/n, E-08193 Bellaterra, Spain

${ }^{11}$ Institut d'Estudis Espacials de Catalunya (IEEC), E-08034 Barcelona, Spain

${ }^{12}$ Landessternwarte, Zentrum für Astronomie der Universität Heidelberg, Königstuhl 12, D-69117 Heidelberg, Germany

${ }^{13}$ Instituto de Astrofísica de Andalucía (CSIC), Glorieta de la Astronomía s/n, E-18008 Granada, Spain

${ }^{14}$ Institut für Astrophysik, Georg-August-Universität, Friedrich-Hund-Platz 1, D-37077 Göttingen, Germany

${ }^{15}$ Centro de Astrobiología (CSIC-INTA), ESAC, Camino bajo del castillo s/n, E-28692 Villanueva de la Cañada, Madrid, Spain

${ }^{16}$ Centro de Astrobiología (CSIC-INTA), Carretera de Ajalvir km 4, E-28850 Torrejón de Ardoz, Madrid, Spain

${ }^{17}$ California Institute of Technology, 1200 E California Blvd, Pasadena, CA 91125, USA

${ }^{18}$ IPAC, 770 South Wilson Avenue, Pasadena, CA 91125, USA

${ }^{19}$ Department of Astronomy, Tsinghua University, Beijing 100084, People's Republic of China

${ }^{20}$ Department of Astronomy and Astrophysics, University of Chicago, 5640 S. Ellis Avenue, Chicago, IL 60637, USA

${ }^{21}$ University of Maryland, Baltimore County, 1000 Hilltop Circle, Baltimore, MD 21250, USA

22 The Adler Planetarium, 1300 South Lakeshore Drive, Chicago, IL 60605, USA

${ }^{23}$ NASA Goddard Space Flight Center, 8800 Greenbelt Road, Greenbelt, MD 20771, USA

${ }^{24}$ NASA Goddard Space Flight Center Sellers Exoplanet Environments Collaboration, 8800 Greenbelt Road, Greenbelt, MD 20771, USA

${ }^{25}$ NASA Goddard Space Flight Center, 800 Greenbelt Road, Greenbelt, MD 20771, USA

${ }^{26}$ University of Maryland Baltimore County, 1000 Hilltop Circle, Baltimore, MD 21250, USA

${ }^{27}$ Sub-department of Astrophysics, Department of Physics, University of Oxford, Oxford OX1 3RH, UK

${ }^{28}$ Institut d'Astrophysique de Paris, CNRS, UMR 7095, Sorbonne Université, 98 bis bd Arago, F-75014 Paris, France

${ }^{29}$ Laboratório Nacional de Astrofísica, Rua Estados Unidos 154, Itajubá, MG 37504-364, Brazil

${ }^{30}$ University of Southern Queensland, West St, Darling Heights, QLD 4350, Australia

${ }^{31}$ Astrobiology Center, 2-21-1 Osawa, Mitaka, Tokyo 181-8588, Japan

32 National Astronomical Observatory of Japan, NINS, 2-21-1 Osawa, Mitaka, Tokyo 181-8588, Japan

${ }^{33}$ Department of Astronomical Science, School of Physical Sciences, The Graduate University for Advanced Studies (SOKENDAI), 2-21-1, Osawa, Mitaka, Tokyo, 181-8588, Japan

${ }^{34}$ Department of Astronomy, Graduate School of Science, The University of Tokyo, 7-3-1 Hongo, Bunkyo-ku, Tokyo 113-0033, Japan

${ }^{35}$ Komaba Institute for Science, The University of Tokyo, 3-8-1 Komaba, Meguro, Tokyo 153-8902, Japan

36 JST, PRESTO, 3-8-1 Komaba, Meguro, Tokyo 153-8902, Japan

${ }^{37}$ Instituto de Astrofísica de Canarias (IAC), E-38205 La Laguna, Tenerife, Spain

${ }^{38}$ University of Hawai'i at Manoa, 2500 Campus Road, Honolulu, HI 96822, USA

${ }^{39}$ Carnegie Earth and Planets Laboratory, 5241 Broad Branch Road, N.W., Washington, DC 20015-1305, USA

${ }^{40}$ Center for Astrophysics, Harvard and Smithsonian, 60 Garden Street, Cambridge, MA 02138, USA

${ }^{41}$ Louisiana State University, 202 Nicholson Hall, Baton Rouge, LA 70803, USA

${ }^{42}$ Westfield High School, 4700 Stonecroft Blvd, Chantilly, VA 20151, USA

${ }^{43}$ NASA Ames Research Center, Moffett Field, CA 94035, USA

${ }^{44}$ SETI Institute, Mountain View, CA 94043, USA

${ }^{45}$ Mikulski Archive for Space Telescopes, 3700 San Martin Drive, Baltimore, MD 21218, USA

${ }^{46}$ Department of Physics and Kavli Institute for Astrophysics and Space Research Massachusetts Institute of Technology, 70 Vassar Street, Cambridge, MA 02139, USA

${ }^{47}$ Astrophysics Science Division, NASA Goddard Space Flight Center, 800 Greenbelt Road, Greenbelt, MD 20771, USA

Received 2021 July 1; revised 2021 September 14; accepted 2021 October 1; published 2021 December 7

\begin{abstract}
We present updated radial-velocity (RV) analyses of the AU Mic system. AU Mic is a young (22 Myr) early-M dwarf known to host two transiting planets $-P_{b} \sim 8.46$ days, $R_{b}=4.38_{-0.18}^{+0.18} R_{\oplus}, P_{c} \sim 18.86$ days, $R_{c}=3.51_{-0.16}^{+0.16} R_{\oplus}$. With visible RVs from Calar Alto high-Resolution search for M dwarfs with Exo-earths with Near-infrared and optical
\end{abstract}


echelle Spectrographs (CARMENES)-VIS, CHIRON, HARPS, HIRES, MINERVA-Australis, and Tillinghast Reflector Echelle Spectrograph, as well as near-infrared (NIR) RVs from CARMENES-NIR, CSHELL, IRD, iSHELL, NIRSPEC, and SPIRou, we provide a $5 \sigma$ upper limit to the mass of AU Mic c of $M_{c} \leqslant 20.13 M_{\oplus}$ and present a refined mass of AU Mic b of $M_{b}=20.12_{-1.57}^{+1.72} M_{\oplus}$. Used in our analyses is a new RV modeling toolkit to exploit the wavelength dependence of stellar activity present in our RVs via wavelength-dependent Gaussian processes. By obtaining near-simultaneous visible and near-infrared RVs, we also compute the temporal evolution of RV "color" and introduce a regressional method to aid in isolating Keplerian from stellar activity signals when modeling RVs in future works. Using a multiwavelength Gaussian process model, we demonstrate the ability to recover injected planets at $5 \sigma$ significance with semi-amplitudes down to $\approx 10 \mathrm{~m} \mathrm{~s}^{-1}$ with a known ephemeris, more than an order of magnitude below the stellar activity amplitude. However, we find that the accuracy of the recovered semi-amplitudes is $\sim 50 \%$ for such signals with our model.

Unified Astronomy Thesaurus concepts: Exoplanets (498)

\section{Introduction}

Characterizing young planetary systems is key to improving our understanding of their formation and evolution. Young transiting systems in particular offer a means to directly probe the radii, and together with masses from precise radial-velocity (RV) measurements, the bulk densities of the planets. RV observations are also crucial to constrain the eccentricity of the orbit to understand the kinematic history and stability of the system. A precision of $20 \%$ for the mass determination is further recommended for enabling detailed atmospheric characterization, particularly for terrestrial-mass planets (Batalha et al. 2019).

Unfortunately, searches for planets orbiting young stars have been limited by stellar activity signals comparable in amplitude to that of typical Keplerian signals. Stellar surface inhomogeneities (e.g., cool spots, hot plages) driven by the dynamic stellar magnetic field rotate in and out of view, leading to photometric variations over time. The presence of such active regions breaks the symmetry between the approaching and receding limbs of the star, introducing RV variations over time as well (Desort et al. 2007). These active regions further affect the integrated convective blueshift over the stellar disk, and will therefore manifest as an additional net red- or blueshift (Meunier \& Lagrange 2013; Dumusque et al. 2014). Various techniques have been introduced to lift the degeneracy between activity- and planetary-induced signals in RV data sets, such as line-by-line analyses (Dumusque 2018; Wise et al. 2018; Cretignier et al. 2020) and Gaussian process (GP) modeling (e.g., Haywood et al. 2014; Grunblatt et al. 2015; LópezMorales et al. 2016; Plavchan et al. 2020; Robertson et al. 2020; Klein et al. 2021; Toledo-Padrón et al. 2021), but such measurements remain challenging due to the sparse cadence of typical RV data sets compared to the activity timescales.

AU Mic is a young (22 Myr; Mamajek \& Bell 2014), nearby ( $\beta$ Pictoris moving group, $\sim 10 \mathrm{pc}$; Gaia Collaboration et al. 2018), and active pre-main-sequence M1 dwarf (Plavchan et al. 2020, hereafter referred to as P20). AU Mic hosts an edge-on debris disk (Pecaut \& Mamajek 2013), and therefore the probability for planets to transit is greater than for other systems. Using photometric observations from TESS (Ricker et al. 2015) in Sector 1 (2018 July 25-August 22), P20 discovered an $\approx 8.46$ day Neptune-size $\left(R_{b}=4.38_{-0.18}^{+0.18} R_{\oplus}\right)$ transiting planet, which was further validated to transit with Spitzer observations (hereafter referred to as AU Mic b). P20 also reported the detection of a single-transit event in the TESS Sector 1 light curve, but did not constrain the period with only an isolated event. With high-cadence RVs from SPIRou, Klein et al. (2021; hereafter referred to as K21) measured the mass of AU Mic b and confirmed it to be consistent with a Neptune- mass planet $\left(M_{b}=17.1_{-4.5}^{+4.7} M_{\oplus}\right)$. With more observations of AU Mic from the TESS extended mission in Sector 27 (2020 July 4-30), Martioli et al. (2021; hereafter referred to as M21) determined AU Mic c to be a smaller Neptune-sized planet $\left(R_{c}=3.51_{-0.16}^{+0.16} R_{\oplus}\right)$ with a period of $\approx 18.86$ days.

In this paper, we present and discuss analyses of several years of multiwavelength RV observations of AU Mic that further elucidates this planetary system. In Section 2, we summarize the visible and near-infrared (NIR) RV observations, as well as photometric observations that are used to inform our RV model. In Section 3, we introduce two joint quasi-periodic Gaussian process kernels that are initial steps in taking into account the expected wavelength dependence of stellar activity through simple scaling relations between wavelengths. We then apply our model to RVs of AU Mic and present the results in Section 4. In Section 5, we assess the sensitivity of our RV model through planet injection and recovery tests. We then briefly discuss the utility of " $R V$ color" between two wavelengths in isolating Keplerian from stellar-activity-induced signals in Section 5.4. We finally note the assumptions and caveats in this work in Section 5.5. A summary of this work is provided in Section 6.

\section{Observations}

\section{1. $R V s$}

Our analyses make use of new and archival high-resolution echelle spectra from a variety of facilities, which are summarized in Table 1. We briefly detail new spectroscopic observations and the corresponding RVs from observing programs primarily intended to characterize the AU Mic planetary system.

\subsubsection{CARMENES}

The Calar Alto high-Resolution search for M dwarfs with Exoearths with Near-infrared and optical echelle Spectrographs (CARMENES) instrument (Quirrenbach et al. 2018) is a pair of two high-resolution spectrographs installed at the $3.5 \mathrm{~m}$ telescope at the Calar Alto Observatory in Spain. The visual (VIS) and nearinfrared (NIR) arms cover a wavelength range of 520-960 nm and $960-1710 \mathrm{~nm}$, with resolving powers of $R=94,600$ and $R=80,400$, respectively. AU Mic was observed 100 times with CARMENES during two different campaigns between 2019 July 14 and October 9, and between 2020 July 19 and November 16, respectively. This last observing period was partially contemporaneous with TESS observations of AU Mic in Sector 27 (2020 July 4-30). One or two exposures of $295 \mathrm{~s}$ were obtained per epoch with typical signal-to-noise ratio $(\mathrm{S} / \mathrm{N})$ larger than $70-100$, and at airmasses larger than 2.5 , due the low decl. of the target at the 
Table 1

A Summary of the RV Data Sets Used in This Work

\begin{tabular}{|c|c|c|c|c|c|c|c|}
\hline Spectrograph/Facility & $\lambda / \Delta \lambda\left[\times 10^{3}\right]$ & $N_{\text {nights }}$ & $N_{\text {used }}$ & $\begin{array}{l}\text { Median } \sigma_{\mathrm{RV}} \\
\left(\mathrm{m} \mathrm{s}^{-1}\right)\end{array}$ & Adopted $\lambda(\mathrm{nm})$ & Pipeline & Comm. Paper \\
\hline HIRES/Keck & 85 & 60 & 41 & 2.6 & 565 & 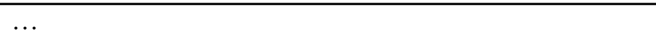 & Vogt et al. (1994) \\
\hline Tillinghast/TRES & 44 & 85 & 55 & 24.2 & 650 & $\ldots$ & Fúrész (2008) \\
\hline $\begin{array}{l}\text { CARMENES-VIS/Calar } \\
\text { Alto } 3.5 \mathrm{~m}\end{array}$ & 94.6 & 63 & 60 & 11.4 & 750 & $\begin{array}{l}\text { caracal (Caballero et al. 2016) serval (Zechmeister } \\
\text { et al. 2018) }\end{array}$ & Bauer et al. (2020) \\
\hline $\begin{array}{l}\text { CARMENES-NIR/Calar } \\
\text { Alto } 3.5 \mathrm{~m}\end{array}$ & 80.4 & 62 & 49 & 32.6 & 1350 & $\ldots$ & $\ldots$ \\
\hline SPIRou/CFHT & 75 & 27 & 27 & 5.0 & 1650 & $\mathrm{~K} 21$ & Donati et al. (2018) \\
\hline iSHELL/IRTF & 85 & 46 & 31 & 5.0 & 2350 & pychell Cale et al. (2019) & Rayner et al. (2016) \\
\hline HARPS-S/La Silla $3.6 \mathrm{~m}$ & 115 & 34 & 0 & 2.2 & 565 & $\begin{array}{l}\text { ESO DRS (Lo Curto et al. 2010) HARPS-TERRA } \\
\text { (Anglada-Escudé \& Butler 2012) }\end{array}$ & Mayor et al. (2003) \\
\hline MINERVA- Australis-T3 & 80 & 13 & 0 & 9.5 & 565 & (Anglada-Escudé \& Butler 2012) & $\begin{array}{l}\text { Wittenmyer et al. (2018), Addison et al. } \\
(2019,2021)\end{array}$ \\
\hline MINERVA- Australis-T4 & 80 & 13 & 0 & 9.5 & 565 & $\ldots$ & $\ldots$ \\
\hline MINERVA- Australis-T6 & 80 & 13 & 0 & 9.5 & 565 & $\ldots$ & $\ldots$ \\
\hline CHIRON/CTIO & 136 & 12 & 0 & 46 & 565 & Piskunov \& Valenti (2002), Cale et al. (2019) & Tokovinin et al. (2013) \\
\hline IRD/Subaru & 70 & 6 & 0 & 3.0 & 1350 & IRAF; (Tody 1993) Hirano et al. (2020b) & Kotani et al. (2018) \\
\hline NIRSPEC/Keck & 25 & 14 & 0 & 50 & 2350 & Bailey et al. (2012) & McLean et al. (1998) \\
\hline CSHELL/IRTF & 36 & 21 & 0 & 26 & 2350 & Gagné et al. (2016), Gao et al. (2016) & Greene et al. (1993) \\
\hline
\end{tabular}

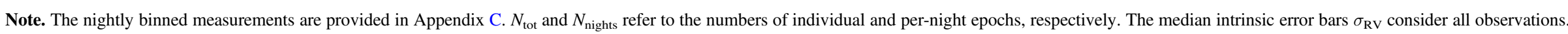
All RV measurements are provided in Table 7. 

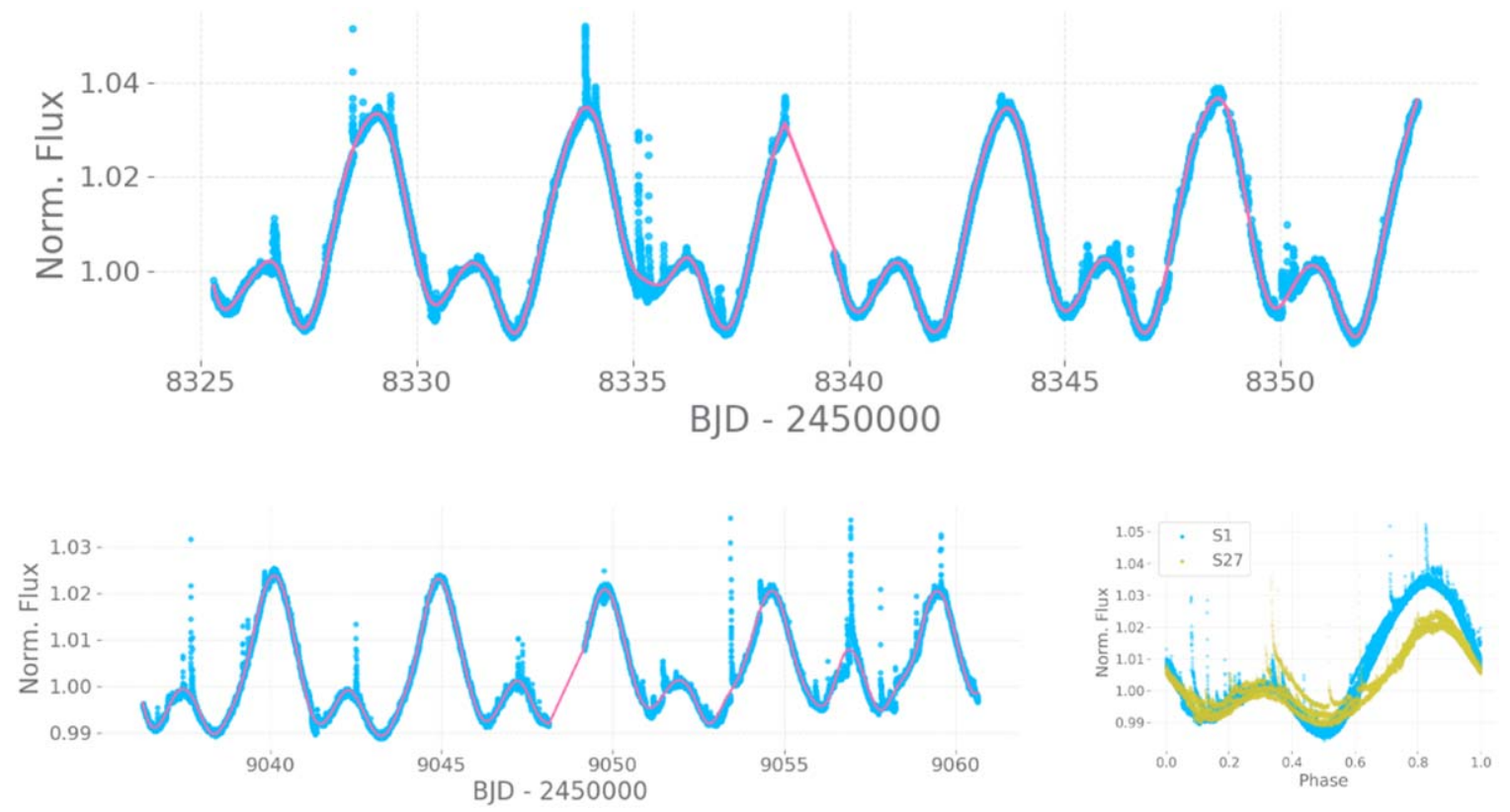

Figure 1. The TESS PDCSAP light curves of AU Mic from Sectors 1 (top) and 27 (bottom). The lower right plot shows both sectors phased to 4.836 days. Although the two seasons exhibit nearly identical periodic signals, Sector 27 exhibits moderate evolution. The least-squares cubic spline fit for each sector is shown in pink.

Calar Alto observatory. CARMENES data were processed by the caracal pipeline (Caballero et al. 2016), which includes bias, flat-field, and dark correction, tracing the echelle orders on the detector, optimal extraction of the one-dimensional spectra, and performance of the initial wavelength calibration using U-Ar, U-Ne, and Th-Ne lamps. The RVs were obtained with the serval pipeline (Zechmeister et al. 2018) by cross-correlating the observed spectrum with a reference template constructed from all observed spectra of the same star. In addition, the serval pipeline also computes the correction for barycentric motion, secular acceleration, instrumental drift using simultaneous observations of FabryPérot etalons, and nightly zero points using RV standards observed during the night (Trifonov et al. 2018).

\subsubsection{CHIRON}

We obtained 14 nightly observations of AU Mic with the CHIRON spectrometer (Kotani et al. 2018) on the SMARTS $1.5 \mathrm{~m}$ telescope at the Cerro Tololo Inter-American Observatory (CTIO) between UT dates 2019 September 14 and November 10 . Observations are recorded in narrow slit mode $(R \sim 136,000)$ using the iodine cell to simultaneously calibrate for the wavelength scale and instrument profile. Like iSHELL observations (see Cale et al. 2019), exposure times ( $\left.t_{\exp }\right)$ were limited to 5 minutes due to the uncertainties of barycenter corrections scaling as $t_{\exp }^{2}$ (Tronsgaard et al. 2019), and the dynamicity of telluric absorption over a single exposure. We initially recorded 22 exposures per night, and later increased this to 42 because the cumulative $\mathrm{S} / \mathrm{N}$ within a night was insufficient $(\sim 100) .{ }^{48}$ Raw CHIRON observations are reduced via the REDUCE package

\footnotetext{
${ }^{48}$ Unlike iSHELL (and like many modern echelle spectrographs), CHIRON makes use of an exposure meter in order to calculate the proper (flux-weighted) exposure midpoint, and therefore longer exposure times will be impacted less by the uncertainty in computing the exposure midpoint. Further, tellurics at visible wavelengths are far more sparse than for iSHELL at $K$-band wavelengths. We therefore recommend significantly longer exposure times $(\geqslant 30$ minutes) for future observations of $\mathrm{AU}$ Mic (or targets of similar brightness) with $\mathrm{CHIRON}$ in narrow slit mode.
}

(Piskunov \& Valenti 2002), and the corresponding RVs are computed using pychell. Unfortunately, a significant fraction of the extracted one-dimensional spectra are too noisy to robustly measure the precise $\mathrm{RVs}$ from (peak $\mathrm{S} / \mathrm{N} \approx 20-30$ per spectral pixel). We therefore flag clear outliers in the RV measurements, and recompute the nightly (binned) RVs, resulting in 12 epochs to be included in our analyses.

\subsubsection{HIRES}

We include 60 Keck-HIRES (Vogt et al. 1994) observations of AU Mic in our analyses. The majority of these observations took place in the second half of 2020, with several nights yielding contemporaneous observations with other facilities. Exposure times range from 204 to $500 \mathrm{~s}$, yielding a median $\mathrm{S} / \mathrm{N} \approx 234$ at $550 \mathrm{~nm}$ per spectral pixel. HIRES spectra are processed and RVs computed via methods described in Howard et al. (2010).

\subsubsection{MINERVA-Australis}

Spectroscopic observations of AU Mic were carried out using the MINERVA-Australis facility situated at the Mount Kent Observatory in Queensland, Australia (Wittenmyer et al. 2018; Addison et al. 2019, 2021) between 2019 July 18 and November 5. MINERVA-Australis consists of an array of four independently operated $0.7 \mathrm{~m}$ CDK700 telescopes, three of which were used in observing AU Mic. Each telescope simultaneously feeds stellar light via fiber optic cables to a single KiwiSpec R4-100 highresolution $(R \sim 80,000)$ spectrograph (Barnes et al. 2012) with wavelength coverage from 480 to $620 \mathrm{~nm}$. In total, we obtained 31 observations with telescope 3 (M-A Tel3), 35 observations with telescope 4 (M-A Tel4), and 33 observations with telescope 6 (M-A Tel6). Exposure times for these observations were set to $1800 \mathrm{~s}$, providing an $\mathrm{S} / \mathrm{N}$ between 15 and 35 per spectral pixel. $\mathrm{RVs}$ are derived for each telescope by using the least-squares shift and fit technique (Anglada-Escudé \& Butler 2012), where the template being matched is the mean spectrum of each 


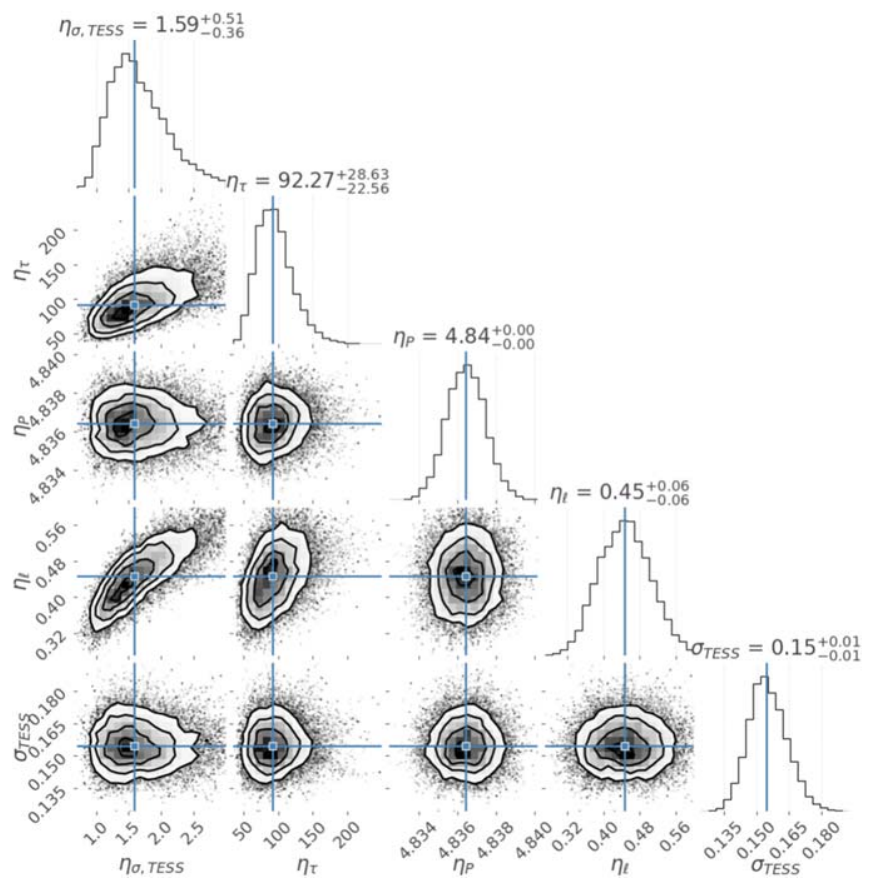

Figure 2. Posterior distributions from fits to the predicted RV variability from the $F F^{\prime}$ technique (Equation (5)).

telescope. Spectrograph drifts are corrected for using simultaneous thorium-argon (ThAr) arc lamp observations.

\subsubsection{TRES}

We include 85 observations (archival and new) of AU Mic observed with the Tillinghast Reflector Echelle Spectrograph (TRES; Fúrész 2008) in our analyses. The majority of these observations took place in the second half of 2019, with several nights yielding contemporaneous observations with other facilities. Typical exposure times range from 600 to $1200 \mathrm{~s}$, with a median $\mathrm{S} / \mathrm{N} \approx 60$ per resolution element. Spectra are processed using methods outlined in Buchhave et al. (2010) and Quinn et al. (2014), with the exception of the cross-correlation template, for which we use the high-S/N median observed spectrum.

\subsubsection{IRD}

We obtained near-infrared, high-resolution spectra of AU Mic using the InfraRed Doppler (IRD) instrument (e.g., Kotani et al. 2018) on the Subaru $8.2 \mathrm{~m}$ telescope. The observations were carried out between 2019 June and October, and we obtained a total of 430 frames with integration times of 30-60 s. Half of these frames were taken on the transit night (UT 2019 June 17), with the goal of measuring the stellar obliquity for AU Mic b, whose RVs were already presented in Hirano et al. (2020a). The raw data are reduced in a standard manner using our custom code as well as IRAF (Tody 1993), and the extracted one-dimensional spectra are analyzed by the RVanalysis pipeline for IRD as described in Hirano et al. (2020b). The typical precision of the derived RVs is $9-13 \mathrm{~m} \mathrm{~s}^{-1}$.

\subsection{7. iSHELL}

We obtained 46 out-of-transit observations of AU Mic with iSHELL on the NASA Infrared Telescope Facility (Rayner et al. 2016) from 2016 to 2020 October. The exposure times varied from 20 to $300 \mathrm{~s}$, and the exposures were repeated 2-23 times within a night to reach a cumulative $S / N$ per spectral pixel $>200$ (the approximate center of the blaze for the middle order, $2.35 \mu \mathrm{m}$ ) for most nights. Raw iSHELL spectra are processed in pychell using methods outlined in Cale et al. (2019).

The corresponding iSHELL RVs are computed in pychell using updated methods to those described in Cale et al. (2019). Instead of starting from an unknown (flat) stellar template, we start with a BT-Settl (Allard et al. 2012) stellar template with $T_{\text {eff }}=3700 \mathrm{~K}$, and with solar values for $\log g$ and $\mathrm{Fe} / \mathrm{H}$. We further Doppler-broaden the template using the rotBroad routine from PyAstronomy (Czesla et al. 2019) with $v \sin i=8.8 \mathrm{~km} \mathrm{~s}^{-1}$. Qualitatively, this broadened template matches the iSHELL observations well. We also "iterate" the template by co-adding residuals in a quasi-inertial reference frame with respect to the star according to the barycenter velocities $\left(v_{\mathrm{BC}}\right)$. However, the stellar RVs for subsequent iterations tend to be highly correlated with $v_{\mathrm{BC}}$ and exhibit significantly larger scatter than the first iteration suggests. We therefore use RVs from the first iteration only, and leave the cause of this correlation as a subject for future work.

\subsection{Photometry from TESS}

The NASA TESS mission (Ricker et al. 2015) observed AU Mic in Sectors 1 (2018 July 25-August 22) and 27 (2020 July 4-30). We download the light curves from the Mikulski Archive for Space Telescopes (MAST; Swade et al. 2018). We use the Science Processing Operations Center (SPOC; Jenkins et al. 2016) "Presearch Data Conditioning" light curves utilizing "Simple Aperture Photometry" (PDCSAP; Smith et al. 2012; Stumpe et al. 2012, 2014) to inform our model in Section 3.3.1.

\section{Radial-velocity Fitting}

\subsection{Bayesian Inference for Radial Velocities}

We primarily seek to utilize a global (joint) Gaussian process model with multiple realizations that give rise to the data we observe with all of the above instruments simultaneously. To implement our desired framework, we have developed two Python packages. We leave the description of optimize-a high-level Bayesian inference framework to Appendix B.

To provide RV-specific routines, we extend the optimize package within the orbits submodule of the pychell (Cale et al. 2019) package. ${ }^{49}$ We define classes specific for RV data, models, and likelihoods, with much of the "boilerplate" code handled through optimize. A top-level "RVProblem" further defines a pool of RV-specific methods for pre- and postoptimization routines, such as plotting phased RVs, periodogram tools, model comparison tests, and propagation of Markov-Chain Monte Carlo (MCMC) chains for deterministic Keplerian parameters (e.g., planet masses, semimajor axes, and densities).

\subsection{Two Chromatic Gaussian Processes}

A Gaussian process kernel is defined through a square matrix, $\boldsymbol{K}$ (also called the covariance matrix), where each entry describes the covariance between two measurements. ${ }^{50} \mathrm{We}$

\footnotetext{
49 Documentation: https://pychell.readthedocs.io/en/latest/.

${ }^{50}$ See Haywood (2015) for a thorough discussion of Gaussian processes.
} 


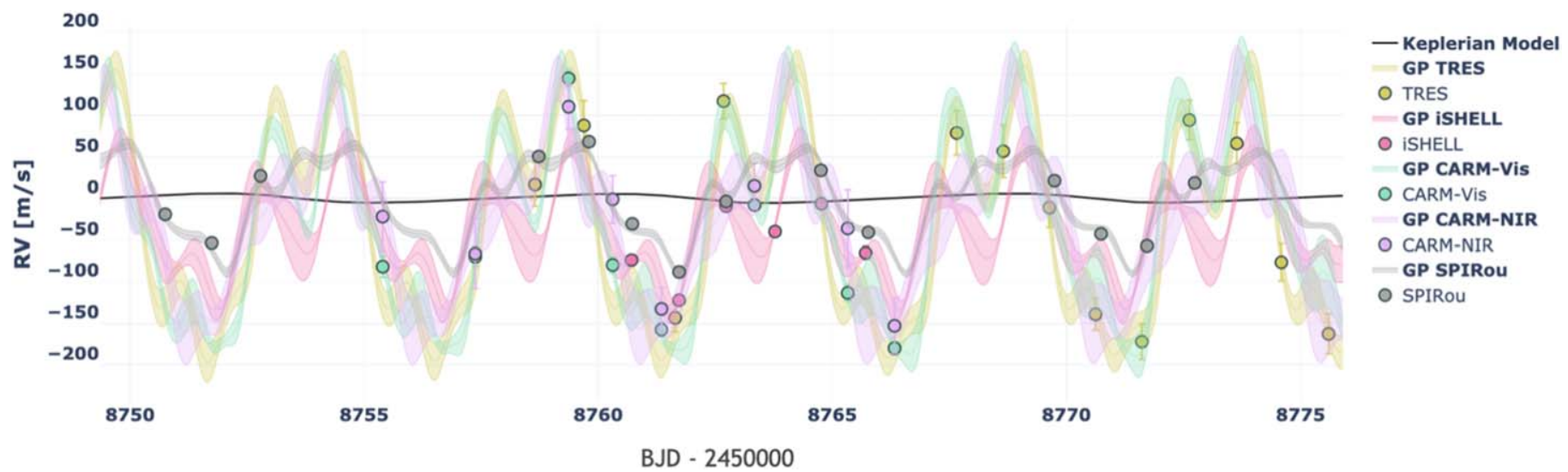

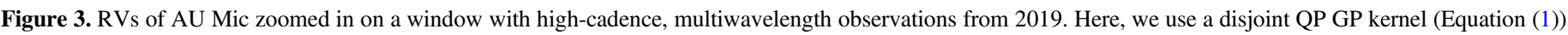

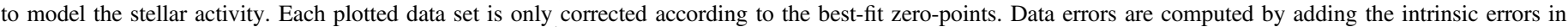

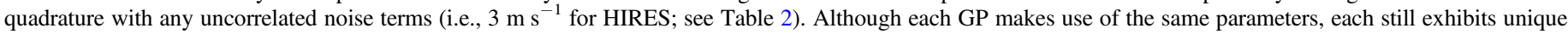

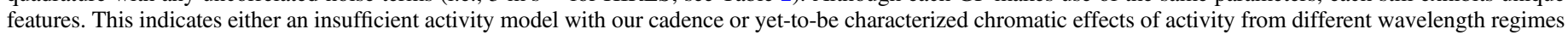
not consistent with a simple scaling relation.

introduce two GP kernels as extensions of the quasi-periodic (QP) kernel, which has been demonstrated in numerous cases to model rotationally modulated stellar activity in both photometric and RV observations (see Section 1): ${ }^{51}$

$$
\boldsymbol{K}_{\mathrm{QP}}\left(t_{i}, t_{j}\right)=\eta_{\sigma}^{2} \exp \left[-\frac{\Delta t^{2}}{2 \eta_{\tau}^{2}}-\frac{1}{2 \eta_{\ell}^{2}} \sin ^{2}\left(\pi \frac{\Delta t}{\eta_{p}}\right)\right]
$$

where $\Delta t=\left|t_{i}-t_{j}\right|$.

Here, $\eta_{P}$ typically represents the stellar-rotation period; $\eta_{\tau}$ the mean spot lifetime; $\eta_{\ell}$ is the relative contribution of the periodic term, which may be interpreted as a smoothing parameter (larger is smoother); and $\eta_{\sigma}$ is the amplitude of the autocorrelation of the activity signal.

We seek to use a fully inclusive QP-like kernel that accounts for the wavelength-dependence of the stellar activity present in our multiwavelength data set. In this work, we only modify the amplitude parameter, $\eta_{\sigma}$; we leave further chromatic modifications (namely, convective blueshift and limb-darkening; see Section 1) as subjects for future work. To first order, we expect the amplitude from activity to be linearly proportional to frequency (or inversely proportional to wavelength). This approximation is a direct result of the spot-contrast scaling with the photon frequency (or inversely with wavelength) from the ratio of two blackbody functions with different effective temperatures (Reiners et al. 2010).

We first reparameterize the amplitude through a linear kernel as follows:

$$
\boldsymbol{K}_{\boldsymbol{J} 1}\left(t_{i}, t_{j}\right)=\eta_{\sigma, \mathrm{s}(i)} \eta_{\sigma, \mathrm{s}(j)} \times \exp [\ldots] .
$$

Here, $\eta_{\sigma, s(i)} \eta_{\sigma, s(j)}$ are the effective amplitudes for the spectrographs at times $t_{i}$ and $t_{j}$, respectively, where $s(i)$ represents an indexing set between the observations at time $t_{i}$ and spectrograph $s .{ }^{52}$ Each amplitude is a free parameter.

We also consider a variation of this kernel that further enforces the expected inverse relationship between the

\footnotetext{
51 Other parameterizations are also common.

52 Truly simultaneous measurements (i.e., $t_{i}=t_{j}$ ) would necessitate a more sophisticated indexing set.
}

amplitude with wavelength. We rewrite the kernel to become:

$$
\boldsymbol{K}_{J 2}\left(t_{i}, t_{j}, \lambda_{i}, \lambda_{j}\right)=\eta_{\sigma, 0}^{2}\left(\frac{\lambda_{0}}{\sqrt{\lambda_{i} \lambda_{j}}}\right)^{2 \eta_{\lambda}} \times \exp [\ldots] .
$$

Here, $\eta_{\sigma, 0}$ is the effective amplitude at $\lambda=\lambda_{0}$, and $\eta_{\lambda}$ is an additional power-law scaling parameter with wavelength to allow for a more flexible nonlinear (with frequency) relation. $\lambda_{i}$ and $\lambda_{j}$ are the "effective" wavelengths for observations at times $t_{i}$ and $t_{j}$, respectively. For both Equations (2) and (3), the expression within square brackets is identical to that in Equation (1).

To make predictions from $\boldsymbol{K}_{\boldsymbol{J} 2}$ (Equation (3)), we follow Rasmussen \& Williams (2006; their Equations (2.23) and (2.24)). We construct the matrix $\boldsymbol{K}_{J 2}\left(t_{i, *}, t_{j}, \lambda_{*}, \lambda_{j}\right)$, which denotes the $n_{*} \times n$ matrix of the covariances evaluated at all pairs of test points and training points (the data). Wavelengths in the ${ }^{*}$ dimension are identical, and therefore each realization corresponds to a unique wavelength. This formulation allows us to realize the GP with high accuracy for all wavelengths so long as at least one wavelength is sampled near $t_{i, *}$. Predictions with kernel $\boldsymbol{K}_{\boldsymbol{J}}$ (Equation (2)) are found in a similar fashion, where each realization corresponds to a particular spectrograph.

\subsection{Primary RV Analyses}

We first bin out-of-transit RV observations from each night (per spectrograph). While not negligible, we expect changes from rotationally modulated activity to be small within a night, so we choose to mitigate activity on shorter timescales our model is not intended to capture (e.g., p-mode oscillations, granulation). The median RV for each spectrograph is also subtracted. We choose to ignore poorly sampled regions with respect to our adopted mean spot lifetime $\eta_{\tau}$ (100 days; see Section 3.3.1). Each instance of a covariance matrix represents a family of functions, and therefore the GP regression may be too flexible (and thus poorly constrained) in regions of lowcadence observations. We also ignore regions with only lowprecision measurements (median errors $\gtrsim 10 \mathrm{~m} \mathrm{~s}^{-1}$ ). This limits our analyses to all observations between 2019 September and 2020 December, and the spectrographs HIRES, TRES, CARMENES-VIS and NIR, SPIRou, and iSHELL. We do 

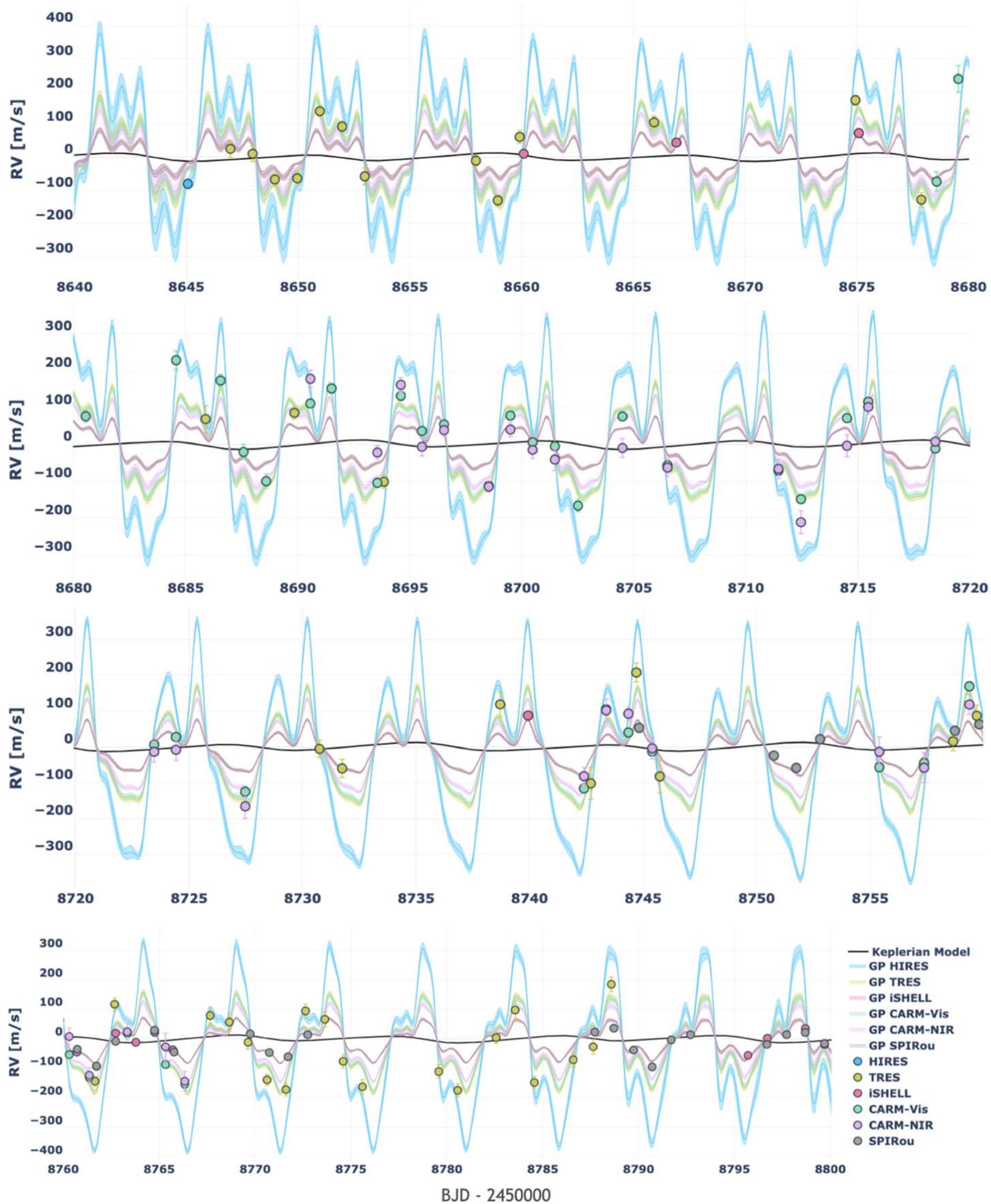

Figure 4. The 2019 RVs using kernel $\boldsymbol{K}_{\boldsymbol{J} 1}$ (Equation (2)) to model the stellar activity. Although there is only one HIRES observation in early 2019 , we are still able to make predictions for the HIRES GP for the entire baseline by using joint kernels. 

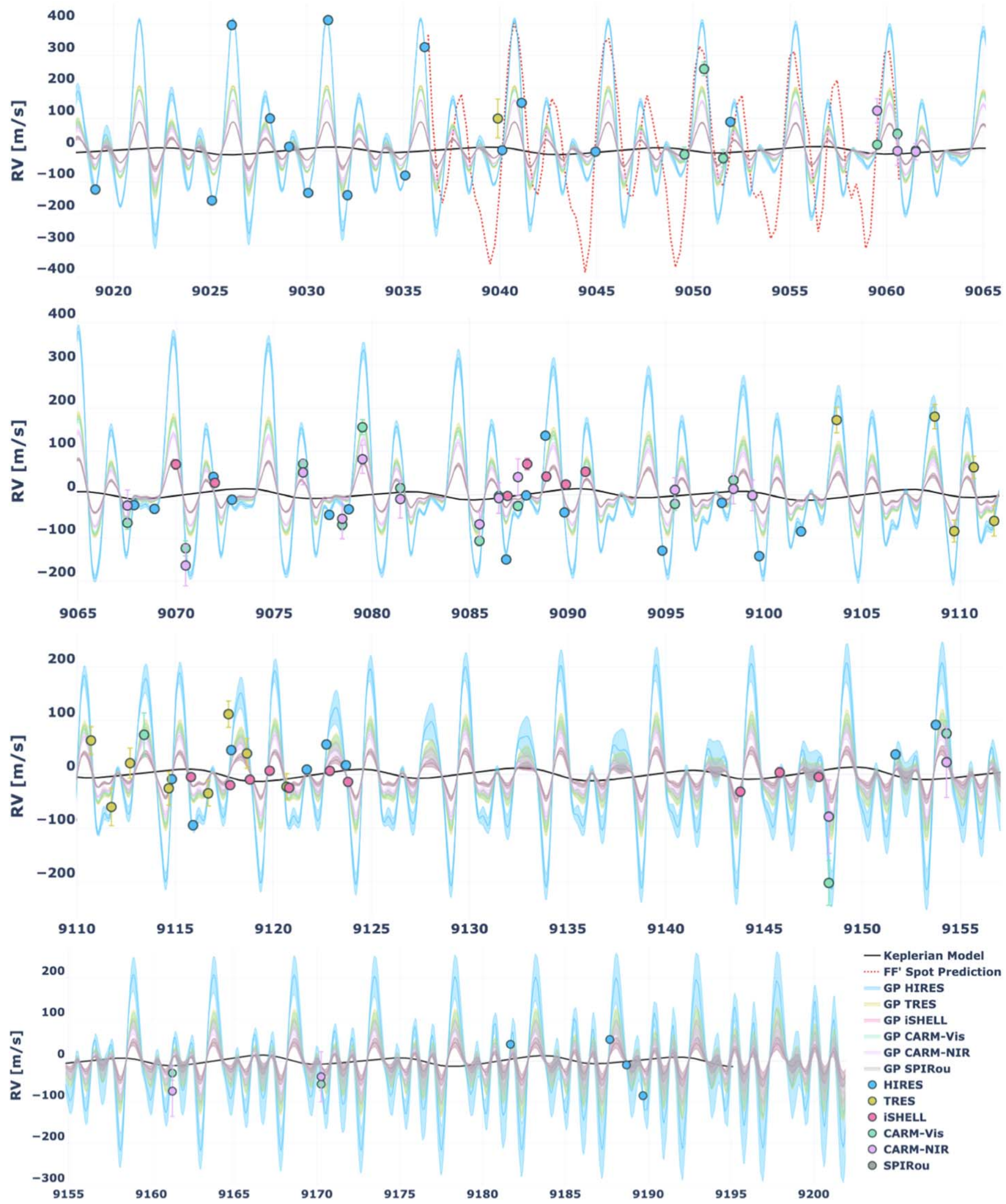

BJD - 2450000

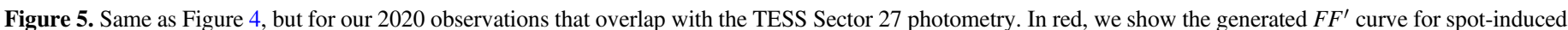
activity signals (Equation (5), arbitrarily scaled) generated from the TESS light curve (Section 3.3.1). 
Table 2

The Model Parameters and Prior Distributions Used in Our Primary Fitting Routines

\begin{tabular}{|c|c|c|c|}
\hline Parameter [units] & Initial Value $\left(P_{0}\right)$ & Priors & Citation \\
\hline$P_{b}$ [days] & 8.4629991 & $\theta$ & Primary transit; M21 \\
\hline $\mathrm{TC}_{b}$ [days] & 2458330.39046 & O & Primary transit;M21 \\
\hline$e_{b}$ & 0.189 & $\mathcal{N}\left(P_{0}, 0.04\right)$ & Secondary eclipse; K. L. Collins et al. (2021, in preparation) \\
\hline$\omega_{b}[\mathrm{rad}]$ & 1.5449655 & $\mathcal{N}\left(P_{0}, 0.004\right)$ & Secondary eclipse; K. L. Collins et al. (2021, in preparation) \\
\hline$K_{b}\left[\mathrm{~m} \mathrm{~s}^{-1}\right]$ & 8.5 & Positive & $\mathrm{K} 21$ \\
\hline$P_{c}$ [days] & 18.858991 & Q & Primary transit; M21 \\
\hline $\mathrm{TC}_{c}$ [days] & 2458342.2243 & $\vec{\theta}$ & Primary transit; M21 \\
\hline$e_{c}$ & 0 & $\overline{0}$ & $\cdots$ \\
\hline$\omega_{c}[\mathrm{rad}]$ & $\pi$ & 0 & $\ldots$ \\
\hline$K_{c}\left[\mathrm{~m} \mathrm{~s}^{-1}\right]$ & 5 & Positive & M21 \\
\hline$\eta_{\sigma, 0}\left[\mathrm{~m} \mathrm{~s}^{-1}\right]$ & 216 & $\mathcal{J}(1,600), \mathcal{N}\left(P_{0}, 10\right)$ & RVs; this work \\
\hline$\eta_{\lambda}$ & 1.18 & $\mathcal{U}(0.3,2)$ & RVs; this work \\
\hline$\eta_{\sigma, \mathrm{HIRES}}\left[\mathrm{m} \mathrm{s}^{-1}\right]$ & 130 & $\mathcal{J}(1,600), \mathcal{N}\left(P_{0}, 30\right)$ & RVs; this work \\
\hline$\eta_{\sigma, \mathrm{TRES}}\left[\mathrm{m} \mathrm{s}^{-1}\right]$ & 103 & $\mathcal{J}(1,600), \mathcal{N}\left(P_{0}, 30\right)$ & RVs; this work \\
\hline$\eta_{\sigma, \mathrm{CARM}-\mathrm{VIS}}\left[\mathrm{m} \mathrm{s}^{-1}\right]$ & 98 & $\mathcal{J}(1,600), \mathcal{N}\left(P_{0}, 30\right)$ & RVs; this work \\
\hline$\eta_{\sigma, \text { CARM-NIR }}\left[\mathrm{m} \mathrm{s}^{-1}\right]$ & 80 & $\mathcal{J}(1,600), \mathcal{N}\left(P_{0}, 30\right)$ & RVs; this work \\
\hline$\eta_{\sigma, \text { SPIRou }}\left[\mathrm{m} \mathrm{s}^{-1}\right]$ & 42 & $\mathcal{J}(1,600), \mathcal{N}\left(P_{0}, 30\right)$ & RVs; this work \\
\hline$\eta_{\sigma, \text { SSHELL }}\left[\mathrm{m} \mathrm{s}^{-1}\right]$ & 40 & $\mathcal{J}(1,600), \mathcal{N}\left(P_{0}, 30\right)$ & RVs; this work \\
\hline$\eta_{\tau}$ [days] & 100 & 0 & TESS light curve and RVs; this work \\
\hline$\eta_{\ell}$ & 0.28 & $\cdots$ & TESS light curve and RVs; this work \\
\hline$\eta_{p}$ [days] & 4.836 & $\mathcal{N}\left(P_{0}, 0.001\right)$ & TESS light curve; this work \\
\hline$\gamma($ per spectrograph $)\left[\mathrm{m} \mathrm{s}^{-1}\right]$ & 1 & $\mathcal{U}(-300,300), \mathcal{N}(0,100)$ & RVs; this work \\
\hline$\sigma_{\text {HIRES }}\left[\mathrm{m} \mathrm{s}^{-1}\right]$ & 3 & $\theta$ & $\ldots$ \\
\hline$\sigma_{\mathrm{TRES}}\left[\mathrm{m} \mathrm{s}^{-1}\right]$ & 0 & $\vec{\theta}$ & $\ldots$ \\
\hline$\sigma_{\text {CARM-VIS }}\left[\mathrm{m} \mathrm{s}^{-1}\right]$ & 0 & 0 & $\ldots$ \\
\hline$\sigma_{\text {CARM-NIR }}\left[\mathrm{m} \mathrm{s}^{-1}\right]$ & 0 & 0 & $\ldots$ \\
\hline$\sigma_{\text {SPIRou }}\left[\mathrm{m} \mathrm{s}^{-1}\right]$ & 0 & 0 & $\ldots$ \\
\hline$\sigma_{\text {iSHELL }}\left[\mathrm{m} \mathrm{s}^{-1}\right]$ & 0 & 0 & $\ldots$ \\
\hline$M_{\star}\left[M_{\odot}\right]$ & $0.5_{-0.03}^{+0.03}$ & $\ldots$ & $\mathrm{P} 20$ \\
\hline$R_{b}\left[R_{\oplus}\right]$ & $4.38_{-0.18}^{+0.18}$ & $\ldots$ & $\mathrm{P} 20$ \\
\hline$R_{c}\left[R_{\oplus}\right]$ & $3.51_{-0.16}^{+0.16}$ & $\cdots$ & M21 \\
\hline
\end{tabular}

Note. Here, $\boldsymbol{Q}$ indicates the parameter is fixed. We run models utilizing $\boldsymbol{K}_{\boldsymbol{J} 1}$ and $\boldsymbol{K}_{\boldsymbol{J} 2}$. We list the radii of AU Mic b and c measured in M21 that we use to compute the corresponding densities of each planet.

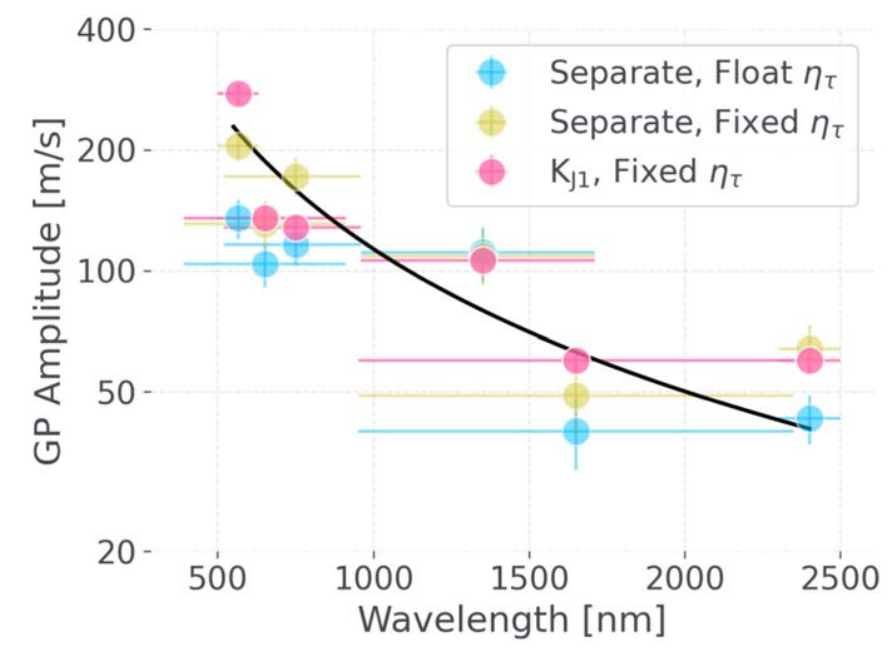

Figure 6. The best-fit GP amplitudes and uncertainties from kernels without enforcing any dependence with wavelength. We consider cases that let $\eta_{\tau}$ and $\eta_{\ell}$ float, as well as our fixed values (see Table 2). The solid line is a leastsquares solution to the amplitudes for kernel $\boldsymbol{K}_{\boldsymbol{J} 2}$ (Equation (3)) for the jointkernel fixed case (pink markers). Horizontal bars correspond to the adopted spectral range for each instrument. not include six binned IRD or 13 binned MINERVA-Australis observations in our primary analyses, as we expect the offsets to be poorly constrained in the presence of stellar activity. Finally, we discard three CARMENES-VIS and 13 CARMENES-NIR measurements from our analyses, primarily near the beginning of each season, due to residuals $>100 \mathrm{~m} \mathrm{~s}^{-1}$ that are inconsistent with our other data sets. We suspect that telluric contamination, which is further exacerbated by the high airmass of the observations, may have degraded the CARMENES observations. For completeness, we present fit results including all spectrographs in Appendix D. A summary of measurements is provided in Table 1.

Our RV model first consists of two Keplerian components for the known transiting planets, a GP model for stellar activity, and per-instrument zero points. The zero points are each assigned to $1 \mathrm{~m} \mathrm{~s}^{-1}$ with a uniform prior of $\pm 300 \mathrm{~m} \mathrm{~s}^{-1}$. We further adopt a normal prior of $\mathcal{N}(0,100)$ to make each offset well-behaved. When using multiple priors, the composite prior probability for such a parameter will not integrate to unity. For the combination of a uniform + normal prior, this is not a concern; the normal prior is properly normalized and takes on a continuous range of values, whereas the uniform prior will either result in a constant term added 

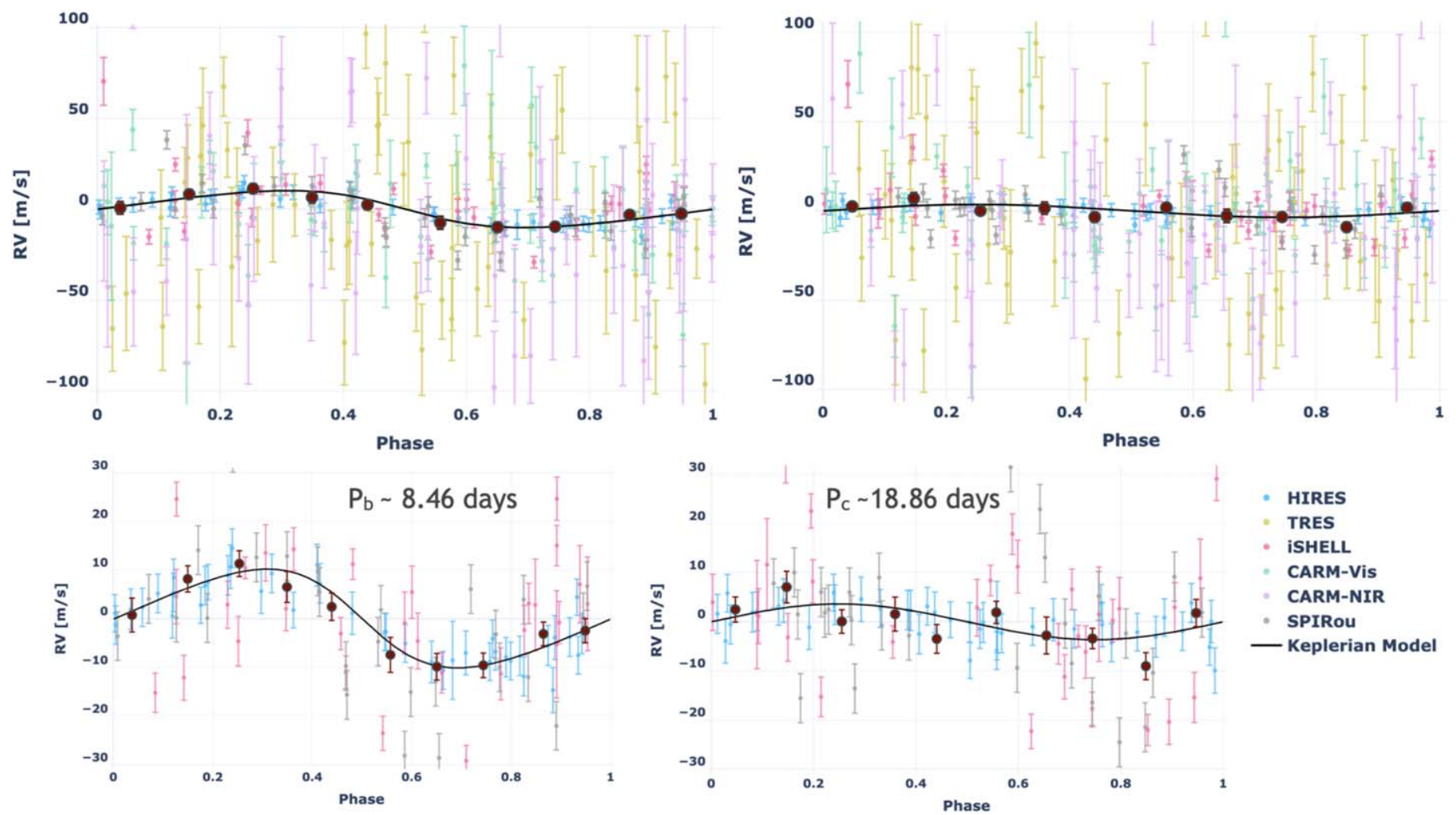

- TRES

- ISHELL

- CARM-Vis

- CARM-NIR

- SPIRou

Keplerian Model

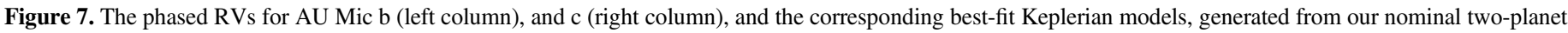

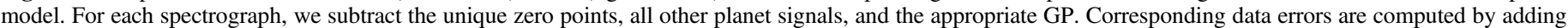

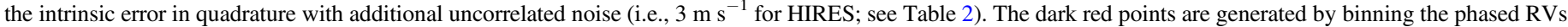

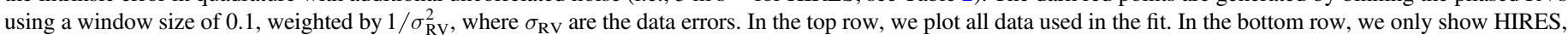

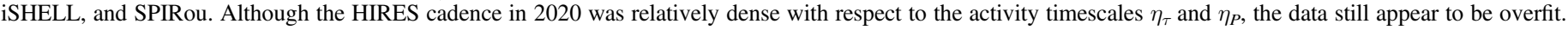

to the likelihood function if the parameter is in bounds, or $-\infty$ if out of bounds.

Analyses of the TESS transits in M21 found $P_{b}=$ $8.4629991 \pm 0.0000024$ days, $\quad \mathrm{TC}_{b}=2458330.39046 \pm$ $0.00016, \quad P_{c}=18.858991 \pm 0.00001$ days, and $\mathrm{TC}_{c}=$ $2458342.2243 \pm 0.0003$. For all of our analyses, we fix $P$ and TC for planets $\mathrm{b}$ and $\mathrm{c}$; the uncertainties in these measurements are insignificant even for our full baseline of $\approx 17 \mathrm{yr}$. The semi-amplitudes of each planet start at $K_{b}=8.5$ $\mathrm{m} \mathrm{s}^{-1}$ and $K_{c}=5 \mathrm{~m} \mathrm{~s}^{-1}$, and are only enforced to be positive. Preliminary analyses of a secondary eclipse observed in Spitzer observations support a moderately eccentric orbit for AU Mic b, with $e_{b}=0.189 \pm 0.04$ (K. I. Collins et al. 2021, in preparation), which is somewhat larger than the eccentricity determined from the duration of the primary transits observed with TESS $\left(e_{b}=0.12 \pm 0.04\right.$; Gilbert et al. 2021). We assume a circular orbit for AU Mic c, and further examine eccentric cases in Section 5.1. The Keplerian component of our RV model in pychell is nearly identical to that used in RadVel (Fulton et al. 2018). Kepler's equation is written in Python and makes use of the numba. anjit decorator (Lam et al. 2015) for optimal performance. We exclusively use the orbit basis $\{P$, $\mathrm{TC}, e, \omega, K\}$.

Our optimizer seeks to maximize the natural logarithm of the a posteriori probability (MAP) under the assumption of normally distributed errors:

$$
\ln \mathcal{L}=-\frac{1}{2}\left[\boldsymbol{r}^{\top} \boldsymbol{K}_{\boldsymbol{o}}^{-1} \boldsymbol{r}+\ln \left|\boldsymbol{K}_{\boldsymbol{o}}\right|+N \ln (2 \pi)\right]+\sum_{i} \ln \mathcal{P}_{i}
$$

Here, $\boldsymbol{r}$ is the vector of residuals between the observations and model, $\boldsymbol{K}_{\boldsymbol{o}}$ is the covariance matrix sampled at the same observations, $N$ is the number of data points, and $\left\{\mathcal{P}_{i}\right\}$ is the set of prior knowledge. We maximize Equation (4) using the iterative Nelder-Mead algorithm described in Cale et al. (2019), which is included as part of the optimize package. We also sample the posterior distributions using the emcee package (Foreman-Mackey et al. 2013) for a subset of models to determine parameter uncertainties, always starting from the MAP-derived parameters. In all cases, we use twice the number of chains as varied parameters. We perform a burn-in phase of 1000 steps followed by a full MCMC analysis for $\approx 50 \times$ the median autocorrelation time (steps) of all chains.

\subsubsection{Estimation of Kernel Parameters}

We briefly analyze both sectors of TESS photometry in order to estimate the GP kernel parameters $\eta_{\tau}$, $\eta_{\ell}$, and $\eta_{P}$. We note that the rotationally modulated structure in both sectors is consistent (Figure 1). If we assume spots are spatially static in the rest frame of the stellar surface (i.e., spots do not migrate), this suggests a similar spot configuration and contrast for each sector. We first determine $\eta_{P}$ by qualitatively analyzing both TESS sectors phased up to periods close to that used in M21 (4.862 \pm 0.032 days) with a step size of 0.001 day (see Figure 1 ). We find $\eta_{P} \approx 4.836$ or $\eta_{P} \approx 4.869$ days from our range of periods tested; no periods between these two values are consistent with our assumption of an identical spot configuration. The difference in these two periods further corresponds to 


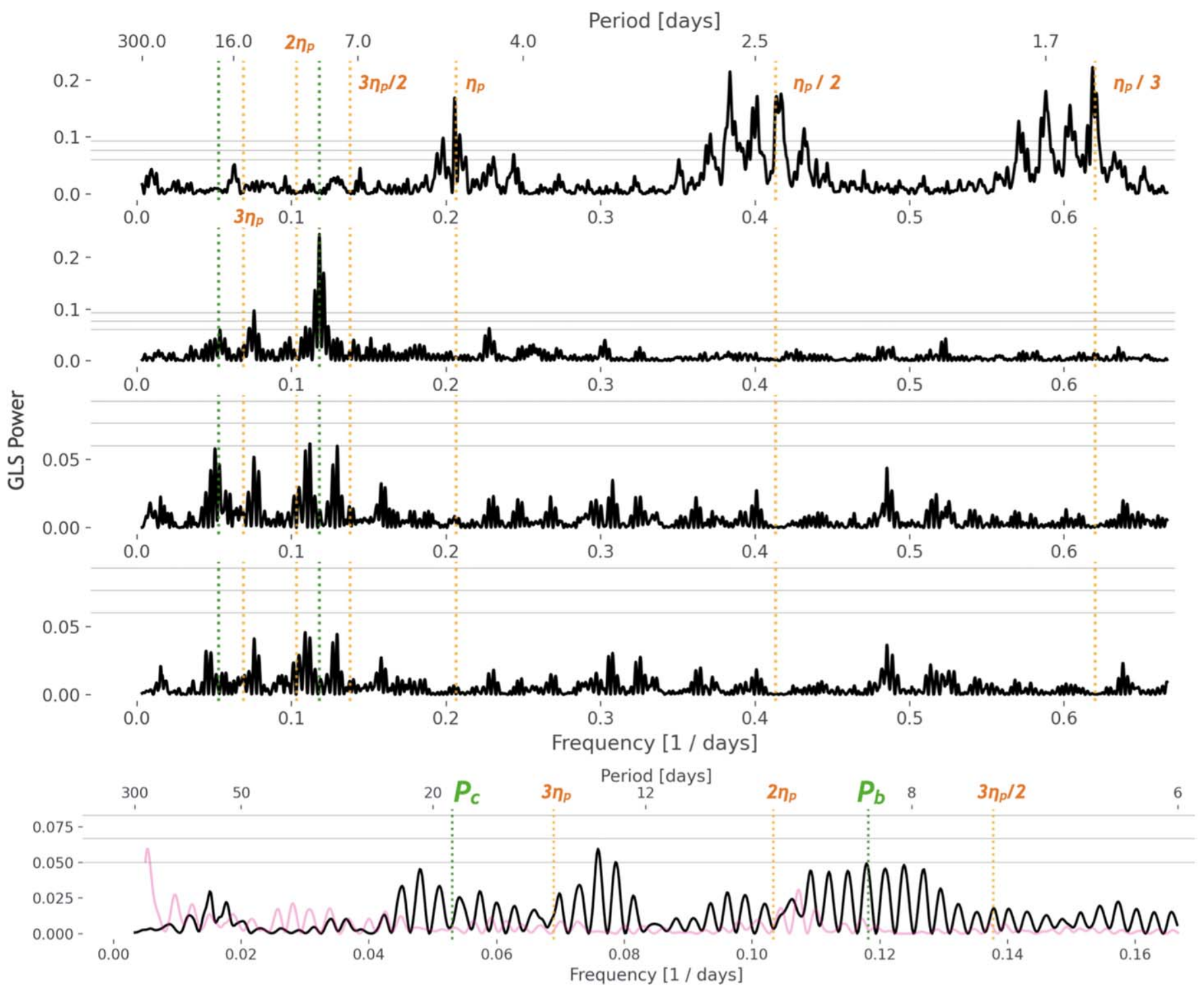

Figure 8. GLS periodograms for AU Mic. Rows 1-4 are generated from our nominal two-planet MAP fit result using $\boldsymbol{K}_{\boldsymbol{J} 1}$ (Equation (2)) to model the stellar activity. From top to bottom, with each step applying an additional "correction," we show: (1) zero-point corrected RVs, (2) activity-filtered RVs, (3) planet b-filtered RVs, and (4) planet c-filtered RVs. Annotated from left to right in green are the periods for AU Mic c and b. In the top row, we also annotate in orange (from left to right) potential aliases of the stellar rotation period $3 \eta_{P}, 2 \eta_{P}$, and $3 \eta_{P} / 2$, followed by the first three harmonics. In the bottom row, we compute a periodogram from an activity-filtered and trend-corrected zero-planet model to indicate how power from planets is absorbed by the GP. In each periodogram, we also identify the FAP power levels corresponding to $0.1 \%$ (highest), $1 \%$, and $10 \%$ (lowest). The clear alias present in all periodograms is caused from the large gap between the two seasons of observations. In the bottom panel, we also plot in pink a Lomb-Scargle periodogram (arbitrarily scaled) of our window function (i.e., identical yet arbitrary RVs at each observation).

one additional period between the two sectors (i.e., $\mid 1 / \eta_{P, 1}-$ $1 / \eta_{P, 2} \mid \approx 1 / 700$ day $^{-1}$ ). The smaller of these two values implies $\mathrm{AU}$ Mic $\mathrm{b}$ is in a 7:4 resonance with the stellar rotation period (Szabó et al. 2021), potentially indicating tidal interactions between the planet and star. We adopt $\eta_{P} \sim \mathcal{N}(4.836,0.001)$ in all our analyses where the uncertainty is a conservative estimate determined by our step size.

Although the TESS light curve itself can provide insight into $\eta_{\tau}$ and $\eta_{\ell}$, we instead try to estimate these values directly from the predicted spot-induced RV variability via the $F F^{\prime}$ technique (Aigrain et al. 2012):

$$
\Delta \mathrm{RV}_{\text {spots }}(t)=-F(t) F^{\prime}(t) R_{\star} / f .
$$

Here, $F$ is the photometric flux and $f$ represents the relative flux drop for a spot at the center of the stellar disk. To compute $F$ and $F^{\prime}$ (the derivative of $F$ with respect to time), we first fit the TESS light curve via cubic spline regression (scipy. interpolate. LSQUnivariateSpline; Virtanen et al. 2020) for each sector individually, with knots sampled in units of 0.5 day $(\approx 10 \%$ of one rotation period), to average over transits and the majority of flare events (Figure 1). The nominal cubic splines are then used to directly compute both $F$ and $F^{\prime}$ on a downsampled grid of 100 evenly spaced points for each sector. We then divide the resulting joint-sector curve by its standard deviation for normalization; we do not care to directly fit for the chromatic parameter $f$ (TESS, ...). We further assume $f$ to be constant in time (i.e., spots are welldispersed on the stellar surface). We then perform both MAP and MCMC analyses for this curve using a standard QP kernel (Equation (1)) with loose uniform priors of $\eta_{\tau} \sim \mathcal{U}(10,2000)$ (days) and $\eta_{\ell} \sim \mathcal{U}(0.05,0.6)$. We set the intrinsic error bars of the curve to zero but include an additional "jitter" (white noise) 


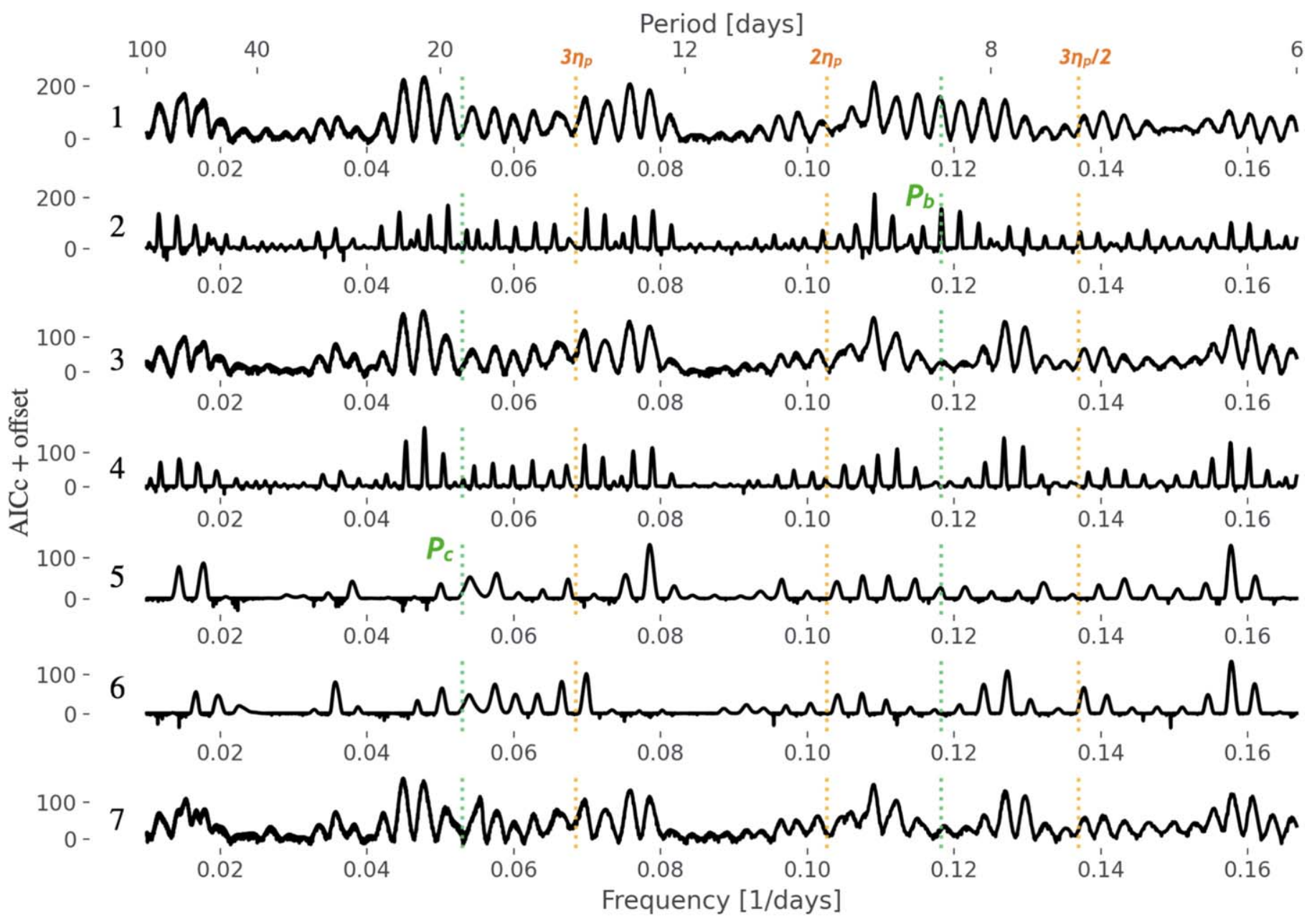

Figure 9. "Brute-force" periodograms for AU Mic with different assumptions for planetary models, but all making use of kernel $\boldsymbol{K}_{\boldsymbol{J} 1}$ (Equation (2)) to model the stellar activity. In each row, we perform a MAP fit for a wide range of fixed periods for a particular "test" planet. In row 1, we include no other planets in our model, and allow for the test planet's TC to float. In row 2, we perform the same search but fixing TC to the nominal value for AU Mic b (Table 2). In row 3 , we include a model for AU Mic b (with $K_{b} \sim \mathcal{N}(8.5,2.5)$; see K21), and search for a second planet, again letting TC float. In rows 4-6, we perform the same search but fix the test planet's TC to one of the three times of transit for AU Mic c from TESS (in chronological order). In the bottom row, we include nominal models for AU Mic b and $\mathrm{c}$ $\left(K_{b} \sim \mathcal{N}(8.5,2.5), K_{c}>0\right)$. We also annotate the same potential aliases with the stellar rotation period (orange) and planetary periods (green) as in Figure 8 .

term in the model with a Jeffreys prior (Jeffreys 1946) distribution with the knee at zero to help keep the jitter well-behaved by discouraging larger values unless it significantly improves the fit quality through an inversely proportional penalty term. The amplitude of the model is drawn from a wide uniform distribution of $\mathcal{U}(0.3,3.0)$. The posterior distributions are provided in Figure 2.

A fit to the $F F^{\prime}$ curve suggests a mean activity timescale $\eta_{\tau} \approx 92_{-23}^{+29}$ days. Although our interpretation implies $\eta_{\tau}$ should be comparable to the gap between the two sectors, $(\sim 700$ days), we do not have photometric measurements between the two sectors, and therefore cannot speak to evolution that will be important for our 2019 observations. We further note that the TESS Sector 27 light curve exhibits moderate evolution whereas Sector 1 appears more stable (Figure 1). The posterior distributions are also consistent with a relatively smooth GP with the period length scale $\eta_{\ell} \approx 0.45 \pm 0.06$.

Before making use of our joint kernels, we first assess the performance of the standard QP kernel (Equation (1)) for each instrument individually. Here, each spectrograph makes use of a unique QP kernel and amplitude term, but the remaining three GP parameters are shared across all kernels. Each amplitude is drawn from a normal prior with mean equal to the standard deviation of the data set, and a conservative width of $30 \mathrm{~m} \mathrm{~s}^{-1}$. The expected semi-amplitudes for AU Mic b and $\mathrm{c}\left(\lesssim 10 \mathrm{~m} \mathrm{~s}^{-1}\right)$ will negligibly affect this estimation. We also apply a Jeffreys prior with the knee at zero to help keep the amplitude wellbehaved. ${ }^{53}$ For $\eta_{\tau}$ and $\eta_{\ell}$, we first make use of the same priors used to model the $F F^{\prime}$ curve. We further include a fixed jitter term at $3 \mathrm{~m} \mathrm{~s}^{-1}$ added in quadrature along the diagonal of the covariance matrix $\boldsymbol{K}_{\boldsymbol{o}}$ for the HIRES observations only; HIRES observations provide the smallest intrinsic uncertainties, but are most impacted by activity (largest in amplitude), so we choose to moderately downweight the HIRES observations. Given the flexibility of GP regression with a nightly cadence, we choose not to fit for (nor include) jitter terms for other spectrographs, and further discuss this decision in Section 5.5. This is the most flexible model we employ to the RVs, and we therefore use these results to flag the aforementioned CARMENES-VIS and CARMENES-NIR measurements.

\footnotetext{
53 Although the composite prior for the GP amplitudes is not proper (i.e., does not integrate to unity; see Section 3.3), we find nearly identical results with only the normal prior.
} 


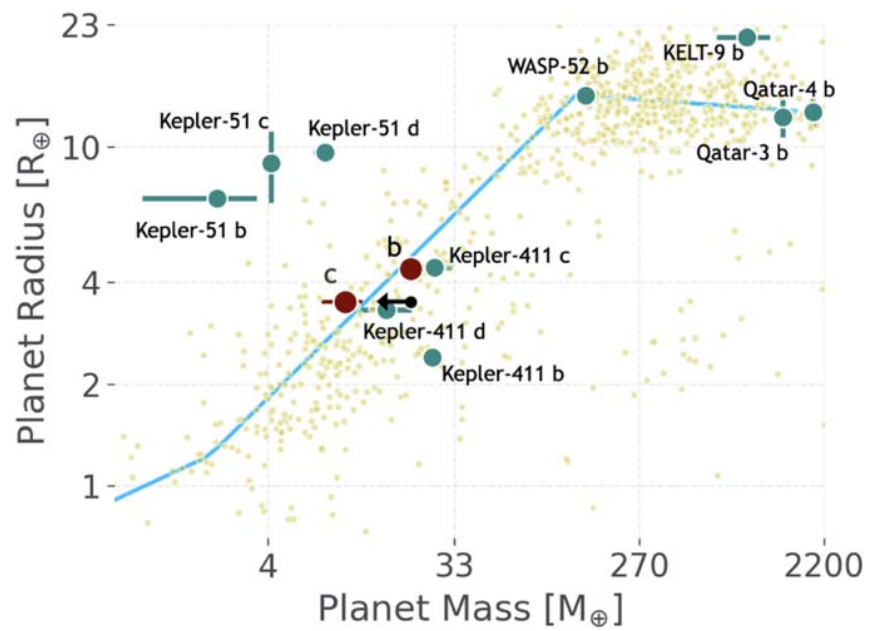

Figure 10. The mass vs. radius for all exoplanets with provided radii and masses from the NASA Exoplanet Archive (2019). For AU Mic b and c, we plot (maroon markers) the masses determined from our two-planet model with kernel $\boldsymbol{K}_{\boldsymbol{J} 1}$. We also indicate with an arrow the $5 \sigma$ upper limit to the mass of AU Mic c determined from the posterior of $K_{c}$. The radii for b and c are those reported in M21. In blue, we plot a piecewise Chen-Kipping mass-radius relation (Chen \& Kipping 2017). We also annotate (cyan markers) the masses and radii for a sample of young planets (estimated stellar age $\lesssim 400 \mathrm{Myr}$ ).

We find normally distributed posteriors for $\eta_{\tau}$ and $\eta_{\ell}$ (Figure 14), but the reduced $\chi^{2}$ statistic of 0.32 indicates the model over fits the data. The per-spectrograph amplitudes are reasonably consistent with their respective priors, so we assert this is a result of $\eta_{\tau}(\approx 43$ days $)$ and/or $\eta_{\ell}(\approx 0.23)$ taking on too small of values, indicating our RV model is insufficient to constrain these values from the RV observations, due to insufficient cadence and/or an inadequate model. We therefore again fix $\eta_{\tau}=100$ days to let each season have mostly distinct activity models, while minimizing the flexibility within each season, which is consistent with what the $F F^{\prime}$ curve suggests. As a compromise between the $F F^{\prime}$ and $\mathrm{RV}$ analyses, we also fix $\eta_{\ell}=0.28$. Our adopted value of $\eta_{\tau}$ is larger than that used in $\mathrm{K} 21\left(\approx 70\right.$ days ${ }^{54}$ ), while $\eta_{\ell}$ is nearly identical. We further explore these decisions and their impact on our derived semi-amplitudes in Section 5.2. With fixed value for $\eta_{\tau}$, we rerun MAP and MCMC fits with disjoint kernels, yielding a reduced $\chi^{2}$ of 0.86 , indicating the model is now only slightly overfit.

\subsubsection{Joint-kernel RV Fitting}

We use results from the disjoint case to inform our primary joint-kernel models. Although the different GPs appear similar (Figure 3), each still exhibits unique features, suggesting a simple scaling is not valid and/or insufficient sampling for each kernel individually. Regardless, our two joint kernels will enforce a perfect scaling between any two spectrographs. The RVs are shown in Figures 4 and 5.

We run MAP and MCMC fits using the joint kernel $\boldsymbol{K}_{\boldsymbol{J} 1}$ (Equation (2)) again making use of the same normal and Jeffreys priors for each amplitude. We then fit the resulting set of best-fit amplitudes using our proposed power-law relation (see Equation (3)): $\eta_{\sigma}(\lambda)=\eta_{\sigma, 0}\left(\lambda_{0} / \lambda\right)^{\eta_{\lambda}}$ with scipy.optimize.curve_fit (Jones et al. 2001; Figure 6). We arbitrarily anchor $\lambda_{0}$ at $\lambda=565 \mathrm{~nm}$. The effective mean wavelength of each spectrograph should consider the RV information content (stellar

\footnotetext{
54 In K21, the hyperparameters $\eta_{\tau}$ and $\eta_{\ell}$ absorb the factors of two present in the formulation used in this work (Equation (1)).
}

and calibration), and ignore regions with dense telluric features. For gas-cell-calibrated spectrographs (HIRES, CHIRON, and iSHELL), we do limit the the range to regions with gas-cell features. For all other spectrographs, we take the effective RV information content to be uniform over the full spectral range as a "zeroth-order" approximation (Reiners \& Zechmeister 2020). We further do not consider regions of tellurics that may have been masked (e.g., CARMENES RVs generated with serval). Although these estimations are imperfect, they are only relevant to kernel $\boldsymbol{K}_{\boldsymbol{J} 2}$ (Equation (3)). The adopted wavelengths for each spectrograph are listed in Table 1.

We find $\eta_{\sigma, 0} \approx 221 \mathrm{~m} \mathrm{~s}^{-1}$, and $\eta_{\lambda} \approx 1.17$. This amplitude is significantly larger than the intrinsic scatter of our observations (namely HIRES) suggests, so we adopt a tight normal prior of $\mathcal{N}(221,10)$ to restrict it from getting any larger. We only apply a loose uniform prior for $\eta_{\lambda} \sim \mathcal{U}(0.2,2)$. We then run corresponding MAP and MCMC fits with kernel $\boldsymbol{K}_{\boldsymbol{J} 2}$ (Equation (3)). A summary of all parameters is provided in Table 2 . We present and discuss fit results from both joint kernels in Section 4.

\section{Results}

The best-fit parameters and corresponding uncertainties from the MAP and MCMC analyses with a two-planet model using joint kernels $\boldsymbol{K}_{\boldsymbol{J} 1}$ (Equation (2)) and $\boldsymbol{K}_{\boldsymbol{J} 2}$ (Equation (3)) are provided in Table 3 . We compute planet masses, densities, and orbital semimajor axes by propagating the appropriate MCMC chains. The uncertainties in $M_{\star}$ and the planetary radii from Table 2 are added in quadrature where appropriate. Corner plots presenting the posterior distributions of each varied parameter are provided in Figures 16 and 17 for kernels $\boldsymbol{K}_{\boldsymbol{J} 1}$ and $\boldsymbol{K}_{\boldsymbol{J} 2}$, respectively. All chains are well-converged, with posteriors resembling Gaussian distributions. We find the offsets for each spectrograph are highly correlated with one another; we note this is unique to the cases leveraging a joint kernel, and strongest when data sets overlap, but we do not further explore this result.

Unlike kernel $\boldsymbol{K}_{\boldsymbol{J} 2}, \boldsymbol{K}_{\boldsymbol{J} 1}$ only enforces a scaling relation between the different spectrographs, but no correlation with wavelength, so we adopt results from $\boldsymbol{K}_{\boldsymbol{J} 1}$ for our primary results, although the results for $K_{b}$ and $K_{c}$ are moderately consistent between kernels. With kernel $\boldsymbol{K}_{\boldsymbol{J} 1}$ (Equation (2)), we report the median semiamplitudes of AU Mic b and $\mathrm{c}$ to be $10.23_{-0.91}^{+0.88} \mathrm{~m} \mathrm{~s}^{-1}$ and $3.68_{-0.86}^{+0.87} \mathrm{~m} \mathrm{~s}^{-1}$, corresponding to masses of $20.12_{-1.72}^{+1.57} M_{\oplus}$ and $9.60_{-2.31}^{+2.07} M_{\oplus}$, respectively. The phased-up RVs for AU Mic b and c are shown in Figure 7. With kernel $\boldsymbol{K}_{\boldsymbol{J} 2}$ (Equation (3)), we find $K_{b}=8.92_{-0.85}^{+0.85} \mathrm{~m} \mathrm{~s}^{-1}$ and $K_{c}=5.21_{-0.87}^{+0.90} \mathrm{~m} \mathrm{~s}^{-1}$. Both our findings for $K_{b}$ are larger but within $1 \sigma$ of the semi-amplitude reported in $\mathrm{K} 21\left(8.5_{-2.2}^{+2.3} \mathrm{~m} \mathrm{~s}^{-1}\right)$. The mass of AU Mic $\mathrm{c}$ is also consistent with a Chen-Kipping mass-radius relation $\left(\approx 12.1 M_{\oplus}\right.$; Chen \& Kipping 2017). The posterior distributions for $e_{b}$ and $\omega_{b}$ are also consistent with their respective priors. Our finding for $K_{b}$ is nearly twice as large as that obtained when using disjoint QP kernels (5.58 $\mathrm{m} \mathrm{s}^{-1}$; Figure 15), although the uncertainties are similar. With disjoint kernels, we find no evidence in the RVs for AU Mic c.

We further validate our results by computing the Bayesian information criterion (BIC) and the small-sample Akaike information criterion (Akaike 1974; Burnham \& Anderson 2002). We compute the relevant quantities for a power set of planet models. We are not trying to independently detect the eccentricity of AU Mic b, and therefore we do not include cases with $e_{b}=0$. Prior probabilities are not included in the calculation of the 
Table 3

Best-fit Parameters and Corresponding Keplerian Variables for Our Primary Two-planet Fits Using Joint Kernels $\boldsymbol{K}_{\boldsymbol{J} 1}$ (Equation (2)) and $\boldsymbol{K}_{\boldsymbol{J} 2}$ (Equation (3))

\begin{tabular}{|c|c|c|c|c|}
\hline Name [units] & MAP (J1) & MCMC (J1) & MAP (J2) & MCMC (J2) \\
\hline$P_{b}$ [days] & 8.4629991 & $\ldots$ & $\ldots$ & $\cdots$ \\
\hline $\mathrm{TC}_{b}$ [days; BJD] & 2458330.39046 & $\cdots$ & $\cdots$ & $\cdots$ \\
\hline$e_{b}$ & 0.187 & $0.186_{-0.035}^{+0.036}$ & 0.182 & $0.181_{-0.035}^{+0.035}$ \\
\hline$\omega_{b}$ [radians $]$ & 1.5452 & $1.5451_{-0.0038}^{+0.0038}$ & 1.5453 & $1.5454_{-0.0041}^{+0.0041}$ \\
\hline$K_{b}\left[\mathrm{~m} \mathrm{~s}^{-1}\right]$ & 10.21 & $10.23_{-0.91}^{+0.88}$ & 8.94 & $8.92_{-0.85}^{+0.85}$ \\
\hline$M_{b}\left[M_{\oplus}\right]$ & 20.14 & $20.12_{-1.72}^{+1.57}$ & 17.66 & $17.73_{-1.62}^{+1.68}$ \\
\hline$a_{b}[\mathrm{au}]$ & 0.0645 & $0.0645_{-0.0013}^{+0.0013}$ & $\cdots$ & $\cdots$ \\
\hline$\rho_{b}\left[\mathrm{~g} \mathrm{~cm}^{-3}\right]$ & 1.32 & $1.32_{-0.20}^{+0.19}$ & 1.16 & $1.16_{-0.18}^{+0.18}$ \\
\hline$P_{c}[$ days $]$ & 18.858991 & $\cdots$ & $\cdots$ & $\cdots$ \\
\hline $\mathrm{TC}_{c}$ [days; BJD] & 2458342.2243 & $\ldots$ & $\ldots$ & $\cdots$ \\
\hline$e_{c}$ & 0 & $\cdots$ & $\cdots$ & $\cdots$ \\
\hline$\omega_{c}$ [radians] & $\pi$ & $\cdots$ & $\ldots$ & $\ldots$ \\
\hline$K_{c}\left[\mathrm{~m} \mathrm{~s}^{-1}\right]$ & 3.62 & $3.68_{-0.86}^{+0.87}$ & 5.23 & $5.21_{-0.87}^{+0.90}$ \\
\hline$M_{c}\left[M_{\oplus}\right]$ & 9.50 & $9.60_{-2.31}^{+2.07}$ & 13.71 & $14.12_{-2.71}^{+2.48}$ \\
\hline$a_{c}[\mathrm{au}]$ & 0.1101 & $0.1101_{-0.002}^{+0.002}$ & $\cdots$ & $\ldots$ \\
\hline$\rho_{c}\left[\mathrm{~g} \mathrm{~cm}^{-3}\right]$ & 1.21 & $1.22_{-0.29}^{+0.26}$ & 1.75 & $1.80_{-0.34}^{+0.31}$ \\
\hline$\gamma_{\text {HIRES }}\left[\mathrm{m} \mathrm{s}^{-1}\right]$ & 2.9 & $4.1_{-57.0}^{+55.6}$ & -19.4 & $-8.7_{-42.9}^{+43.3}$ \\
\hline$\gamma_{\text {TRES }}\left[\mathrm{m} \mathrm{s}^{-1}\right]$ & 11.4 & $12.1_{-27.8}^{+27.4}$ & -0.2 & $9.3_{-38.0}^{+38.4}$ \\
\hline$\gamma_{\text {CARM-VIS }}\left[\mathrm{m} \mathrm{s}^{-1}\right]$ & 3.7 & $4.3_{-26.6}^{+26.0}$ & -12.1 & $-3.4_{-33.9}^{+34.5}$ \\
\hline$\gamma_{\text {CARM-NIR }}\left[\mathrm{m} \mathrm{s}^{-1}\right]$ & 2.6 & $2.9_{-21.8}^{+21.7}$ & -6.8 & $-1.5_{-21.2}^{+21.2}$ \\
\hline$\gamma_{\text {SPIRou }}\left[\mathrm{m} \mathrm{s}^{-1}\right]$ & 5.5 & $5.6_{-12.4}^{+12.3}$ & 0.72 & $5.1_{-17.3}^{+17.8}$ \\
\hline$\gamma_{\text {iSHELL }}\left[\mathrm{m} \mathrm{s}^{-1}\right]$ & -2.8 & $-2.4_{-12.4}^{+12.1}$ & -7.5 & $-4.3_{-12.9}^{+13.0}$ \\
\hline$\eta_{\sigma, 0}\left[\mathrm{~m} \mathrm{~s}^{-1}\right]$ & $\cdots$ & $\cdots$ & 242.4 & $243.1_{-9.1}^{+8.8}$ \\
\hline$\eta_{\lambda}$ & $\cdots$ & $\cdots$ & 0.843 & $0.845_{-0.024}^{+0.024}$ \\
\hline$\eta_{\sigma, \mathrm{HIRES}}\left[\mathrm{m} \mathrm{s}^{-1}\right]$ & 269.4 & $275.7_{-16.4}^{+17.4}$ & $\cdots$ & $\cdots$ \\
\hline$\eta_{\sigma, \mathrm{TRES}}\left[\mathrm{m} \mathrm{s}^{-1}\right]$ & 132.3 & $135.4_{-9.5}^{+10.6}$ & $\cdots$ & $\cdots$ \\
\hline$\eta_{\sigma, \text { CARM-VIS }}\left[\mathrm{m} \mathrm{s}^{-1}\right]$ & 125.1 & $128.2_{-8.2}^{+8.8}$ & $\cdots$ & $\ldots$ \\
\hline$\eta_{\sigma, \text { CARM-NIR }}\left[\mathrm{m} \mathrm{s}^{-1}\right]$ & 103.0 & $105.5_{-8.7}^{+9.1}$ & $\cdots$ & $\cdots$ \\
\hline$\eta_{\sigma, \text { SPIRou }}\left[\mathrm{m} \mathrm{s}^{-1}\right]$ & 58.5 & $60.1_{-4.0}^{+4.3}$ & $\cdots$ & $\cdots$ \\
\hline$\eta_{\sigma, \text { SSHELL }}\left[\mathrm{m} \mathrm{s}^{-1}\right]$ & 58.5 & $60.0_{-3.9}^{+4.2}$ & $\ldots$ & $\ldots$ \\
\hline$\eta_{P}$ [days] & 4.8384 & $4.8384_{-0.0009}^{+0.0008}$ & 4.8376 & $4.8376_{-0.0009}^{+0.0009}$ \\
\hline
\end{tabular}

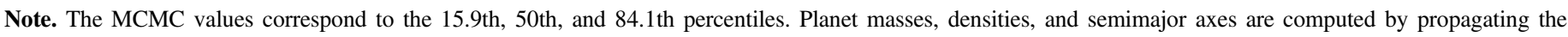
appropriate MCMC chains. We also add in quadrature the uncertainties in $M_{\star}$ and planetary radii from Table 2 where relevant.

corresponding $\ln \mathcal{L}$ (Equation (4)), to maintain normalization between different models. The results are summarized in Table 4 and are consistent with the relative precisions for each derived semi-amplitude.

Finally, we compute and present the reduced chi-squared statistic $\left(\chi_{\text {red }}^{2}\right)$ for each spectrograph individually to assess their respective goodness of fit (Table 5). We add in quadrature the intrinsic error bars with any additional uncorrelated noise (i.e., $3 \mathrm{~m} \mathrm{~s}^{-1}$ for HIRES; see Table 2). We find the HIRES observations are moderately overfit $\left(\chi_{\text {red }}^{2}=0.64\right)$, whereas the other spectrographs are underfit. We suspect this is due to the activity amplitude for HIRES being significantly larger than the other spectrographs despite exhibiting a similar overall dispersion. Although we include an additional $3 \mathrm{~m} \mathrm{~s}^{-1}$ white noise term for the HIRES observations, they still yield the smallest overall error bars and therefore are given the most weight in the GP regression. Although a more flexible uncorrelated noise model may yield a more accurate weighting scheme for the different spectrographs (i.e., a varied "jitter" parameter for each spectrograph), we favor the model without them for the variety of reasons discussed in Section 5.5. Finally, we note that we find moderately similar results when not using the HIRES RVs altogether $\left(K_{b}=12.95 \pm 1.1 \mathrm{~m} \mathrm{~s}^{-1}\right.$, $K_{c}=3.5 \pm 1.0 \mathrm{~m} \mathrm{~s}^{-1}$ ).

\subsection{Evidence For Additional Candidates?}

We compute periodograms to further assess the relative statistical confidence of the two transiting planets and to search for other planets in the system. We first compute a series of generalized Lomb-Scargle (GLS; Zechmeister \& Kürster 2018; Czesla et al. 2019) periodograms out to 500 days after removing the nominal zero points, appropriate GPs, and the two planets, all generated using parameters from our nominal two-planet model (Table 3) with kernel $\boldsymbol{K}_{\boldsymbol{J} 1}$ (Equation (2)) to model the stellar activity. We also compute an activity-filtered periodogram from a planet-free model to assess how much the GP model will absorb planetary signals, and inform our interpretation of other peaks present in the periodogram. We further plot the normalized power levels for false-alarm probabilities (FAPs) of $10 \%, 1 \%$, and $0.1 \%$.

We also compute "brute-force" periodograms by performing MAP fits for a wide range of fixed orbital periods for a userdefined "test planet" with various assumptions for other model 
Table 4

Model Information Criterion for AU Mic b and c Using Kernel $\boldsymbol{K}_{\boldsymbol{J} 1}$ (Equation (2)) to Model the Stellar Activity

\begin{tabular}{lccccc}
\hline \hline Planets & $\ln \mathcal{L}$ & $\chi_{\text {red }}^{2}$ & $N$ Free & $\Delta$ AICc & $\Delta$ BIC \\
\hline $\mathrm{b}, \mathrm{c}$ & -1753.1 & 4.73 & 17 & 0 & 0 \\
$\mathrm{~b}$ & -1762.0 & 4.77 & 16 & 15.5 & 12.2 \\
$\mathrm{c}$ & -1816.4 & 5.14 & 14 & 119.8 & 109.8 \\
None & -1828.8 & 5.23 & 13 & 142.4 & 129.13 \\
\hline
\end{tabular}

Table 5

Reduced $\chi^{2}$ for Each Spectrograph from Our Nominal Two-planet Model Using Kernel $\boldsymbol{K}_{\boldsymbol{J} 1}$

\begin{tabular}{lc}
\hline \hline Spectrograph & $\chi_{\text {red }}^{2}$ \\
\hline HIRES & 1.09 \\
TRES & 6.86 \\
CARMENES-VIS & 5.89 \\
CARMENES-NIR & 4.39 \\
SPIRou & 16.70 \\
iSHELL & 22.43
\end{tabular}

Note. Unlike when using quasi-disjoint kernels (Section 3.3.1), we find the model is overall underfit with the joint kernel. We suspect this is primarily due to an inadequate stellar-activity (i.e., a scaling relation is insufficient between spectrographs) and/or the exclusion of per-spectrograph jitter terms, and discuss these details further in Section 5.5.

parameters (see Addison et al. 2021). Given the time complexity of GP regression, we only consider periods out to 100 days. We first run two searches with no other planets in the model, first allowing for the test planet's TC to float, and second fixing TC to the nominal value for AU Mic b (Table 3). We then run searches for a second planet, this time including a planetary model to account for the orbit of $\mathrm{AU}$ Mic b, with $K_{b} \sim \mathcal{N}(8.5,2.5)$, consistent with the semi-amplitude found in K21. We again consider the case of letting the test planet's TC float, then run three cases with fixing the test planet's TC to each time of transit for AU Mic c from the TESS Sector 1 and 27 light curves (M21). Last, we perform a search for a third planet, letting its TC float, and including models for AU Mic b and c $\left(K_{b} \sim \mathcal{N}(8.5,2.5), K_{c}>0\right)$.

Both the GLS (Figure 8) and brute-force (Figure 9) periodograms exhibit clear aliasing with a frequency of $\approx 0.00281$ days $^{-1}$ (or 356 days), which we attribute to having two seasons of observations separated by $\approx 200$ days. Given the respective power of AU Mic b in both the GLS and brute-force planet-free periodograms, we briefly explore other peaks with similar power, even though all other peaks are below all three FAPs after removing the nominal two-planet model (Figure 8, row 3; Figure 9, row 7). Both two- and zero-planet periodograms (as well as GLS and brute-force) show power between the orbits of AU Mic b and c near 12.72 and 13.19 days, as well as power near 66.7 days for the residual RVs. Although these peaks are comparable in power to AU Mic $b$ in both planet-free periodograms, they may be spurious. We further discuss the confirmation of AU Mic b and $\mathrm{c}$ as well as the validation of such additional potential candidates in Section 5.3. A mass-radius diagram is shown in Figure 10 to place the mass and radius of all $\mathrm{AU}$ Mic $\mathrm{b}$ and $\mathrm{c}$ in context with other known exoplanets, including a subset of young sample of exoplanets shown in P20. The plotted masses for AU Mic b and $\mathrm{c}$ are from our nominal two-planet model using kernel $\boldsymbol{K}_{\boldsymbol{J} 1}$ (Equation (2)).
Table 6

MCMC Results with Different Assumptions for the Mean Spot Lifetime $\eta_{\tau}$ and $\eta_{\ell}$ Using Kernel $\boldsymbol{K}_{\boldsymbol{J} 1}$ (Equation (2))

\begin{tabular}{lcccc}
\hline \hline$\eta_{\tau}$ (days) & $\eta_{\ell}$ & $K_{b}\left(\mathrm{~m} \mathrm{~s}^{-1}\right)$ & $K_{c}\left(\mathrm{~m} \mathrm{~s}^{-1}\right)$ & $\chi_{\text {red }}^{2}$ \\
\hline 40 & 0.15 & $8.79 \pm 1.47$ & $7.38 \pm 1.65$ & 1.58 \\
40 & 0.2 & $8.84 \pm 1.30$ & $8.51 \pm 1.33$ & 2.10 \\
40 & 0.25 & $8.23 \pm 1.17$ & $9.05 \pm 1.17$ & 2.45 \\
40 & 0.3 & $7.41 \pm 1.08$ & $9.13 \pm 1.13$ & 2.68 \\
40 & 0.35 & $6.95 \pm 0.98$ & $9.23 \pm 1.05$ & 2.85 \\
\hline 70 & 0.15 & $8.74 \pm 1.24$ & $6.88 \pm 1.30$ & 1.99 \\
70 & 0.2 & $10.32 \pm 1.13$ & $5.90 \pm 1.07$ & 2.69 \\
70 & 0.25 & $10.45 \pm 1.04$ & $4.76 \pm 0.95$ & 3.25 \\
70 & 0.3 & $9.61 \pm 0.91$ & $4.16 \pm 0.89$ & 3.65 \\
70 & 0.35 & $9.18 \pm 0.82$ & $3.94 \pm 0.83$ & 3.90 \\
\hline 100 & 0.15 & $9.28 \pm 1.17$ & $5.88 \pm 1.08$ & 2.46 \\
100 & 0.2 & $10.85 \pm 1.00$ & $4.73 \pm 0.98$ & 3.20 \\
100 & 0.25 & $10.78 \pm 0.95$ & $3.78 \pm 0.87$ & 3.76 \\
100 & 0.3 & $9.81 \pm 0.85$ & $3.63 \pm 0.80$ & 4.12 \\
100 & 0.35 & $9.22 \pm 0.80$ & $3.60 \pm 0.77$ & 4.39 \\
\hline 200 & 0.15 & $9.38 \pm 0.98$ & $4.01 \pm 0.96$ & 3.44 \\
200 & 0.2 & $11.04 \pm 0.89$ & $3.35 \pm 0.84$ & 4.16 \\
200 & 0.25 & $11.09 \pm 0.87$ & $3.38 \pm 0.77$ & 4.73 \\
200 & 0.3 & $10.14 \pm 0.84$ & $4.51 \pm 0.76$ & 5.28 \\
200 & 0.35 & $9.06 \pm 0.73$ & $4.77 \pm 0.66$ & 5.59 \\
\hline 300 & 0.15 & $9.32 \pm 0.92$ & $3.84 \pm 0.87$ & 3.82 \\
300 & 0.2 & $10.60 \pm 0.88$ & $3.78 \pm 0.81$ & 4.68 \\
300 & 0.25 & $10.42 \pm 0.80$ & $4.99 \pm 0.74$ & 5.59 \\
300 & 0.3 & $10.51 \pm 0.76$ & $4.52 \pm 0.68$ & 5.96 \\
300 & 0.35 & $9.89 \pm 0.75$ & $4.41 \pm 0.68$ & 6.27 \\
\hline & & & &
\end{tabular}

Note. For each row, we fix the values of $\eta_{\tau}$ and $\eta_{\ell}$. All other model parameters take on the initial values and priors from Table 2 for a two-planet model. We perform a MAP fit followed by MCMC sampling for each case. We report the nominal values and uncertainties for the semi-amplitudes of AU Mic b and $\mathrm{c}$ from the MCMC fitting, as well as the reduced chi-squared statistic, $\chi_{\text {red }}^{2}$, using the MAP-derived parameters. Uncertainties reported here for $K_{b}$ and $K_{c}$ are the average of the upper and lower uncertainties.

\section{Discussion}

\subsection{Constraints on Eccentricity}

Here, we briefly explore eccentric orbits for the two-transiting planets $\mathrm{b}$ and $\mathrm{c}$. For each planet, we take $e \sim \mathcal{U}(0,0.7)$ and $\omega \sim \mathcal{U}(0,2 \pi)$. We only use kernel $\boldsymbol{K}_{\boldsymbol{J} 1}$ (Equation (2)) to model the stellar activity. Posterior distributions are presented in Figure 18. We find $e_{b}=0.30 \pm 0.04$, which is $\approx 50 \%$ larger than our prior informed by a secondary eclipse event indicates. The corresponding finding of $\omega_{b}=3.01 \pm 0.27$ is also inconsistent with our adopted prior for $\omega_{b}$. The posterior distribution for $e_{c}$ is concentrated at the upper bound (0.7), implying an overlapping orbit with AU Mic b. Orbital stability calculations presented in M21 indicate $e_{c}<0.2$, so we assert our model is unable to accurately constrain its eccentricity. The behavior of $e_{c}$ further indicates our detection of $K_{c}$ may not be significant.

\subsection{Sensitivity to Kernel Hyperparameters}

Our analyses in Section 3.3.1 make use of a fixed mean spot lifetime $\eta_{\tau}=100$ days and smoothing parameter $\eta_{\ell}=0.28$. Here, we determine how sensitive the recovered semi-amplitudes of AU Mic $\mathrm{b}$ and $\mathrm{c}$ are to these two parameters. We consider $\eta_{\tau} \in\{40$, $70,100,200,300\}$ (days), and $\eta_{\ell} \in\{0.15,0.2,0.25,0.3,0.35\}$. 

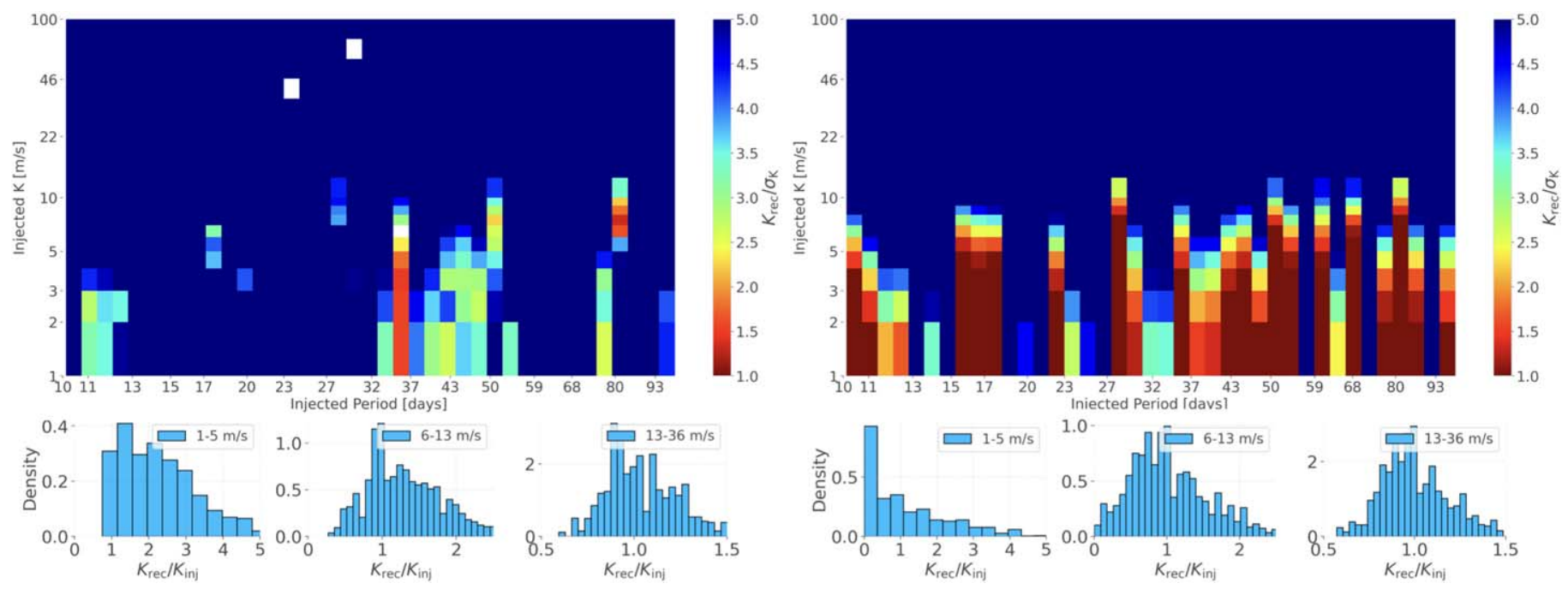

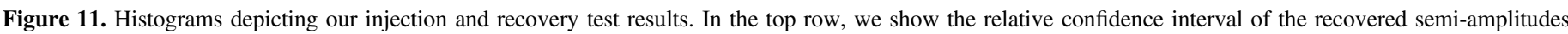

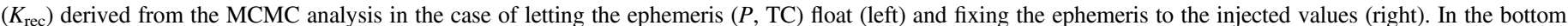
row, we compare the recovered semi-amplitude to the injected value $\left(K_{\mathrm{inj}}\right)$.
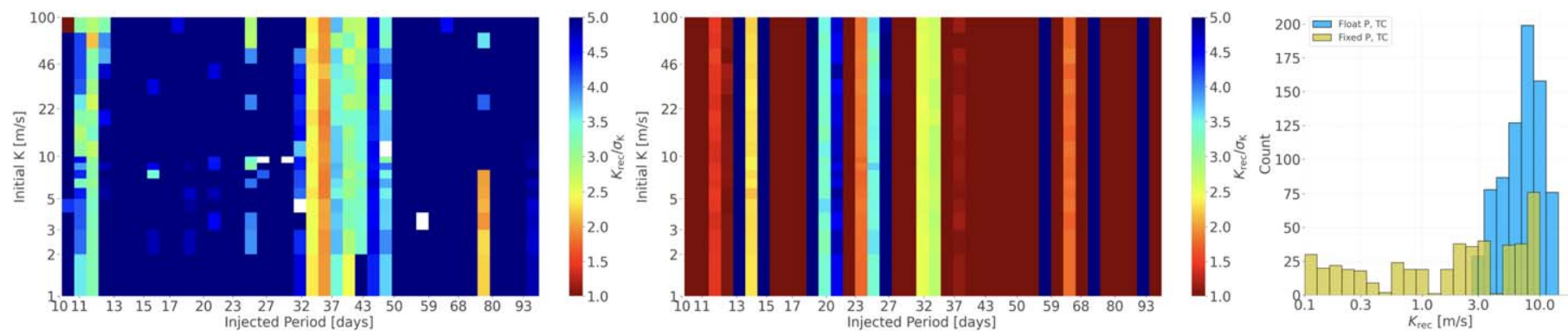

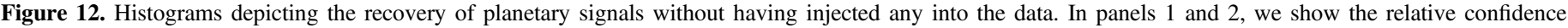

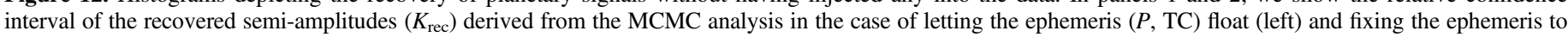
the arbitrary TC $=2457147.36589$ (middle). On the right, we show the recovered semi-amplitudes for each case.

We perform MAP and MCMC fits for all pairs of these two fixed parameters using $\boldsymbol{K}_{\boldsymbol{J} 1}$ (Equation (2)) for a two-planet model. All other parameters adopt initial values and priors from Table 2. Results are summarized in Table 6.

We find $K_{b}$ is only moderately sensitive to the values of each hyperparameter, ranging from $\sim 7$ to $11 \mathrm{~m} \mathrm{~s}^{-1}$. With a larger spot lifetime, $K_{b}$ tends toward larger values, indicating the GP is likely absorbing power from planet $\mathrm{b}$ with a more flexible model (smaller $\eta_{\tau}$ ). However, $K_{b}$ is relatively insensitive to the value of $\eta_{\ell}$. The range of values for $K_{c}$ is larger, changing by nearly a factor of three. Unlike $K_{b}, K_{c}$ is more unstable and tends toward larger values when using a more flexible (smaller) spot lifetime. The reduced chi-squared statistic indicates the model is not overfit in any of the cases performed, but is also larger than unity by a several factors in most cases, indicating our modeling is inadequate.

\subsection{Planet Injection and Recovery}

Here, we assess the fidelity of our RV model applied to the AU Mic system through planetary injection and recovery tests. We first inject planetary signals into the RV data with welldefined semi-amplitudes, periods, and ephemerides (TC). We arbitrarily choose TC $=2457147.36589$ for all injected cases. We consider 40 unique periods between 5.12345 and 100.12345 days, uniformly distributed in $\log$ space. For the semi-amplitude $K$, we consider values from 1 to $10 \mathrm{~m} \mathrm{~s}^{-1}$ with a step size of $1 \mathrm{~m} \mathrm{~s}^{-1}$, as well as values between 10 and 100 $\mathrm{m} \mathrm{s}^{-1}$ that are uniformly distributed in log space (20 total values). In all cases, we include a model for AU Mic b with fixed $P$ and TC such that $K_{b} \sim \mathcal{N}(8.5,2.5)$. We first assess our recovery capabilities using a Gaussian prior for $P$ such that $P \sim \mathcal{N}\left(P_{\text {inj }}, P_{\text {inj }} / 50\right)$ and a uniform prior for TC such that $\mathrm{TC} \sim \mathcal{U}\left(\mathrm{TC}_{\mathrm{inj}} \pm P_{\mathrm{inj}} / 2\right)$. For each injected planet (one at a time), we run our MAP and MCMC analyses to determine the recovered $K$ and corresponding uncertainty. The starting values for $K$ and TC are always the injected values. We also consider the same injection and recovery test but with $P$ and TC fixed to the injected value. We finally determine how susceptible our RV model is to pick out "fake" planets by running these same two trials with no injected planets. Although there are no injected planets, we still run the same trials as for the injected case with different initial values for $K$. A two-dimensional histogram of the recovered $K$ as a fraction of the injected $K$, as well as the associated uncertainty (also as a fraction of the injected $K$ ) for each case are shown in Figures 11 and 12 for the injected and non-injected cases, respectively.

In the case of injected planets, we find our RV model is able to confidently recover semi-amplitudes down to a few $\mathrm{m} \mathrm{s}^{-1}$ in this data set with a relative precision of $\gtrsim 4 \sigma$. However, a closer inspection reveals the recovered semi-amplitudes are typically 


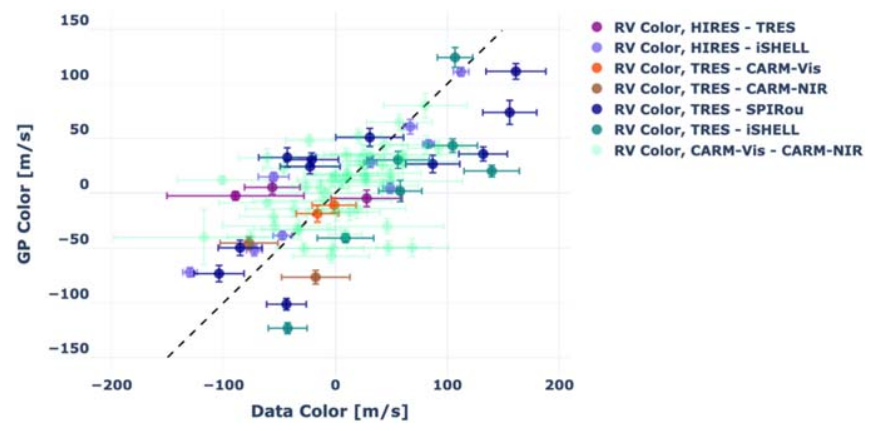

Figure 13. The observed "RV color" $=\operatorname{RV}(t, \lambda)-\operatorname{RV}\left(t, \lambda^{\prime}\right)\left(\lambda^{\prime}>\lambda\right)$ from our 2019 and 2020 nights with nearly simultaneous measurements at unique wavelengths. These are plotted against the same RV-color difference predicted by our chromatic GP model using kernel $\boldsymbol{K}_{\boldsymbol{J} 2}$. Pairs consisting of CARMENESVIS and CARMENES-NIR measurements are nearly transparent, to make other pairs more visible. We do not plot pairs of SPIRou and iSHELL, because they are tightly centered near zero. A dashed one-to-one line is also shown. The weighted coefficient of determination $\left(R^{2}\right)$ is $\approx 0.68$.

larger than the injected $K$, in particular for smaller injected values $\left(1-5 \mathrm{~m} \mathrm{~s}^{-1}\right)$ that include our measured semi-amplitude AU Mic c. When the ephemeris is known, we tend to poorly measure the smallest values of $K$, indicating the recovered TC in the non-fixed case is unlikely what we have injected. In the $6-13 \mathrm{~m} \mathrm{~s}^{-1}$ range, which covers the recovered semi-amplitude of AU Mic b, we find that the accuracy of the recovered semi-amplitudes are $\sim 50 \%$. So, while we quote a formal precision on the mass of AU Mic b to be $M_{b}=20.12_{-1.57}^{+1.72} M_{\oplus}(\sim 9 \%$ precision $)$, our injection and recovery tests indicate that the accuracy on the mass of AU Mic b is only known to a factor of two.

Unfortunately, attempts to recover non-injected planets are "unsuccessful," in that our modeling finds strong evidence for planets we did not inject (Figure 12) in the case of allowing $P$ and TC to float. A deeper investigation into the posteriors of such fits indicates certain parameters (primarily $P$ and TC) are typically not well-behaved and yield non-Gaussian distributions. When fixing $P$ and TC to "nominal" values, our modeling does not tend to find such nonexistent planets (Figure 12).

The confident recoveries of "fake" planets in our tests indicate our GP model is flexible enough to find relatively (quasi)-stable islands in probability space with high confidence for $K$ specifically. Although several peaks stand out in our periodogram analyses (Figures 8 and 9), more observations and/or more sophisticated modeling are needed to robustly claim these periods as statistically validated planets. We further note that the recovered values of $K$ for the smallest injected values are inaccurate, indicating our measurement of $K_{c}=$ $3.68 \mathrm{~m} \mathrm{~s}^{-1}$ is also moderately unconvincing, and is likely an overestimate given the behavior of all recoveries at this level of $K$. We finally note this analysis is limited by planets we do not account for in the model, which may impact our ability to recover certain combinations of $P$ and TC. Further tests using several values for the injected TC may also yield different results. With these limitations in mind, we also provide an estimation of the upper limit to the mass of AU Mic c. We find a $5 \sigma$ upper limit to the semi-amplitude of $\mathrm{AU}$ Mic $\mathrm{c}$ of $\leqslant 7.68 \mathrm{~m} \mathrm{~s}^{-1}$, corresponding to a mass of $\leqslant 20.13 M_{\oplus}$.

\subsection{Utility of $R V$ Color}

Our chromatic kernel used in this work is an initial step to exploit the expected correlation of stellar activity versus wavelength by introducing a scaling relation between wavelengths (Equations (2) and (3)). Here, we examine the "RV color" for our multiwavelength data set in order to further assess the correlation between our RVs with expected activity:

$$
\mathrm{RV}_{\text {color }}\left(t, \lambda, \lambda^{\prime}\right)=\mathrm{RV}(t, \lambda)-\mathrm{RV}\left(t, \lambda^{\prime}\right) \text {. }
$$

We first determine which nights contain nearly simultaneous measurements at unique wavelengths. We require observations to be within 0.3 day ( $\approx 6 \%$ of one rotation period) of each other, in order to minimize differences from rotationally modulated activity but increase the number of pairs for our brief use. For each nearly simultaneous chromatic pair, we compute the "data color" directly from the measured RVs as well as the "GP color" by computing the differences between the two measurements and two GPs sampled at the identical times, respectively (such that $\lambda^{\prime}>\lambda$ ). This calculation requires knowledge of the parameters in order to remove the perinstrument zero points and realize each appropriate GP, so we make use of the MAP-derived parameters in Table 3 with kernel $\boldsymbol{K}_{\boldsymbol{J} 2}$ (Equation (3)). The correlation between the data and GP color is shown in Figure 13. The agreement between the data and the model (weighted $R^{2} \approx 0.71$ ) indicates that our chromatic GP technique is doing a good job of reproducing the $\mathrm{RV}$-color phenomenon for multiple wavelength pairs.

With a sufficient model for stellar activity, we expect the data and GP RV color to match (up to white noise). Therefore, the "RV color" between the data and GP may be used to further constrain (in future analyses) the model (and therefore prevent overfitting) by including an effective L2 regularization penalty as follows:

$$
\ln \mathcal{L}+=-\Lambda \sum_{t} r_{\mathrm{col}}(t)^{2}
$$

Here, $\boldsymbol{r}_{\mathrm{col}}$ is the vector of residuals between the GP and data RV color, $\Lambda>0$ is a tunable hyperparameter whose value is directly correlated with the relative importance and confidence of the stellar activity model, and $+=$ represents the standard "addition assignment" operator. The vector $\boldsymbol{r}_{\text {col }}$ may be computed for all pairs of wavelengths with (nearly) simultaneous measurements, and each pair can make use of identical or unique values of $\Lambda$. We finally note this regularization term is not limited to our assumption of a simple scaling relation, and could also be used in the case of disjoint kernels.

\subsection{Additional Caveats and Future Work}

Kernels $\boldsymbol{K}_{\boldsymbol{J} 1}$ (2) and $\boldsymbol{K}_{\boldsymbol{J} 2}$ (Equation (3)) make use of a scaling relation for stellar activity models at different wavelengths (spectrographs) where each activity model is drawn from a Gaussian process characterized by a covariance matrix utilizing all observations. Using such joint kernels yields fits with larger scatter than cases using disjoint QP kernels (one per spectrograph; Equation (1)). In the latter case, we find that although each of the activity models appear to be "in-phase" with one another, each GP exhibits unique features that are inconsistent with a simple scaling relation (Figure 3). With nightly sampling, it is difficult to determine whether the observed differences between disjoint GPs are indicative of inadequate sampling or an inadequate RV model (activity + planets). Further, all activity models used in this work make use of identical kernel hyperparameters (excluding the amplitude), which may further be an inadequate assumption. We expect the stellar rotation period $\left(\eta_{P}\right)$ to be identical across wavelengths (or nearly so); however, it is not clear whether the mean 
activity timescale $\left(\eta_{\tau}\right)$ or period length scale $\left(\eta_{\ell}\right)$ in particular should be achromatic hyperparameters.

Our work further excluded per-spectrograph uncorrelated "jitter" terms. We suspect this may be the source of our model's ability to find planets we did not inject into the model (Section 5.3), which we defer to future work. The reduced $\chi^{2}$ values in Tables 4 and 6 quantify the degree to which our models do not capture signals from possible additional planets, incorrect values for eccentricity and/or $\omega$, per-spectrograph systematics not included in the formal measurement uncertainties, stellar activity such as p-mode oscillations, convection noise, or longer timescale variations. Therefore, although our specific likelihood function (Equation (4)) assumes normally distributed errors, we choose not to combine any remaining (i.e., unaccounted for by the provided error bars) potentially correlated noise into an additional uncorrelated jitter term to keep our model simple.

More accurately characterizing the masses and orbits of AU Mic b and c may require a more sophisticated stellar activity model and more intensive multiwavelength cadence. Our work further does not make use of activity indicators (e.g., Ca II $\mathrm{H}$ and $\mathrm{K}, \mathrm{H} \alpha$ ) or asymmetries in the cross-correlation function (e.g., the bisector inverse slope (BIS) or differential line width dLW; Zechmeister et al. 2018) to help constrain the activity model (see Rajpaul et al. 2015). The serval pipeline in particular provides a measure of the chromaticity (CRX) for both the CARMENES-VIS and NIR data sets, which we do not use in our modeling. For AU Mic, we expect that each spectrograph is precise enough to resolve first-order chromatic effects within their respective spectral grasp's, which unfortunately will make the formal uncertainties of each spectrograph larger. Further, our QP-based kernels are primarily intended to capture rotationally modulated activity induced by temperature inhomogeneities on the stellar surface. Although the flexibility of disjoint GPs likely captures other rotationally modulated effects such as convective blueshift and limb-darkening, it will not capture short-term activity such as flares. We finally note that more seasons with high-cadence RVs will help mitigate the strong $1 \mathrm{yr}$ alias present in our data set, and will help determine the correct periods for potential non-transiting planets.

\section{Conclusion}

In this work, we have developed two joint Gaussian process kernels that begin to take into account the expected wavelength dependence of stellar activity through a simple scaling relation. We apply our kernels to a data set of AU Mic, which is composed of RVs from multiple facilities, and wavelengths ranging from visible to $K$-band. With our analyses, we report a refined mass of AU Mic b of $M_{b}=20.12_{-1.72}^{+1.57} M_{\oplus}$, and provide a $4.2 \sigma$ mass estimate of the recently validated transiting planet AU Mic c to be $M_{c}=9.60_{-2.31}^{+2.07} M_{\oplus}$, corresponding to a $5 \sigma$ upper limit of $M_{c} \leqslant 20.13 M_{\oplus}$. We also identify additional peaks present in the activity-filtered RVs, but such periods require more evidence for a robust validation, given the overall flexibility of our RV model with an unknown ephemeris.

In Section 5.1, we find our model is unable to robustly constrain the eccentricity for AU Mic b or c. In Section 5.2, we find the derived planetary semi-amplitudes for AU Mic b and $\mathrm{c}$ are moderately sensitive to the choice of kernel parameters, indicating careful attention must be made when interpreting planetary masses with such a flexible model. Through injection and recovery tests in Section 5.3, we further validate our RV model by demonstrating our ability to recover planets down to $\approx 10 \mathrm{~m} \mathrm{~s}^{-1}$ when the orbit's ephemeris is known. However, we find that the accuracy in the recovered semi-amplitudes is $\sim 50 \%$ at $10 \mathrm{~m} \mathrm{~s}^{-1}$. In Section 5.4, we introduce a method to further leverage the "RV color" correlation between the observations and activity model through penalizing the objective function by including an effective $\mathrm{L} 2$ regularization term.

All data processed with pychell (iSHELL and CHIRON) were run on ARGO, a research computing cluster provided by the Office of Research Computing, and the exo computer cluster, both at George Mason University, VA.

We thank all support astronomers, observers, and engineers from all facilities in helping enable the collection of the data presented in this paper.

The authors wish to recognize and acknowledge the very significant cultural role and reverence that the summit of Maunakea has always had within the indigenous Hawaiian community, where the iSHELL, HIRES, IRD, and SPIRou observations were recorded. We are most fortunate to have the opportunity to conduct observations from this mountain.

This work is supported by grants to Peter Plavchan from NASA (awards 80NSSC20K0251 and 80NSSC21K0349), the National Science Foundation (Astronomy and Astrophysics grants 1716202 and 2006517), and the Mount Cuba Astronomical Foundation.

Emily A. Gilbert also wishes to thank the LSSTC Data Science Fellowship Program, which is funded by LSSTC, NSF Cybertraining Grant \#1829740, the Brinson Foundation, and the Moore Foundation; her participation in the program has benefited this work. Emily is thankful for support from GSFC Sellers Exoplanet Environments Collaboration (SEEC), which is funded by the NASA Planetary Science Division's Internal Scientist Funding Model. The material is based upon work supported by NASA under award number 80GSFC21M0002.

This work is partly supported by JSPS KAKENHI grant No. JP18H05439, JST PRESTO grant No. JPMJPR1775, the Astrobiology Center of National Institutes of Natural Sciences (NINS) (grant No. AB031010).

M.T. is supported by JSPS KAKENHI grant Nos. $18 \mathrm{H} 05442,15 \mathrm{H} 02063$, and 22000005.

The authors also with to acknowledge funding from the Agencia Estatal de Investigación del Ministerio de Ciencia e Innovación (AEI-MCINN) under grant PID2019109522GB-C53.

The authors also wish to thank the California Planet Search (CPS) collaboration for carrying out the HIRES observations recorded in 2020 presented in this work.

MINERVA-Australis is supported by Australian Research Council LIEF Grant LE160100001, Discovery Grant DP180100972, Mount Cuba Astronomical Foundation, and institutional partners University of Southern Queensland, UNSW Australia, MIT, Nanjing University, George Mason University, University of Louisville, University of California Riverside, University of Florida, and The University of Texas at Austin.

CARMENES is an instrument at the Centro Astronómico Hispano-Alemán de Calar Alto (CAHA, Almería, Spain). CARMENES is funded by the German Max-PlanckGesellschaft (MPG), the Spanish Consejo Superior de Investigaciones Científicas (CSIC), the European Union 
through FEDER/ERF FICTS-2011-02 funds, and the members of the CARMENES Consortium (Max-Planck-Institut für Astronomie, Instituto de Astrofísica de Andalucía, Landessternwarte Köonigstuhl, Institut de Ciències de l'Espai, Institut für Astrophysik Göttingen, Universidad Complutense de Madrid, Thüringer Landessternwarte Tautenburg, Instituto de Astrofísica de Canarias, Hamburger Sternwarte, Centro de Astrobiología and Centro Astronómico Hispano-Alemán), with additional contributions by the Spanish Ministry of Economy, the German Science Foundation through the Major Research Instrumentation Programme and DFG Research Unit FOR2544 "Blue Planets around Red Stars," the Klaus Tschira Stiftung, the states of Baden-Württemberg and Niedersachsen, and by the Junta de Andalucía.

We acknowledge financial support from the Agencia Estatal de Investigación of the Ministerio de Ciencia, Innovación y Universidades and the ERDF through projects PID2019109522GB-C5[1:4]/AEI/10.13039/501100011033, PGC2018098153-B-C33, and the Centre of Excellence "Severo Ochoa" and "María de Maeztu" awards to the Instituto de Astrofísica de Canarias (CEX2019-000920-S), Instituto de Astrofísica de Andalucía (SEV-2017-0709), and Centro de Astrobiología (MDM-20170737), and the Generalitat de Catalunya/CERCA program.

This paper includes data collected by the NASA TESS mission that are publicly available from the Mikulski Archive for Space Telescopes (MAST). Funding for the TESS mission is provided by NASA's Science Mission Directorate. We acknowledge the use of public TESS data from pipelines at the TESS Science Office and at the TESS Science Processing Operations Center (Jenkins et al. 2016).

Baptiste Klein acknowledges funding from the European Research Council under the European Union's Horizon 2020 research and innovation program (grant agreement No. 865624, GPRV).

Eder Martioli acknowledges funding from the French National Research Agency (ANR) under contract number ANR-18-CE31-0019 (SPlaSH).

Software: pychell (Cale et al. 2019), optimize, ${ }^{55}$ Matplotlib (Hunter 2007), SciPy (Virtanen et al. 2020), NumPy (Harris et al. 2020), Numba (Lam et al. 2015), corner (ForemanMackey et al. 2020), plotly (Inc., 2015), Gadfly Matplotlib theme https://gist.github.com/JonnyCBB/c464d302fefce4 722fe6cf5f461114ea, emcee, (Foreman-Mackey et al. 2013).

\section{Appendix A Posterior Distributions}

Here, we show the posterior distributions for the relevant RV models employed in this work. In each corner plot, blue lines correspond to the 50th percentile of the distribution. Upper and lower uncertainties correspond to the 84.1st and 15.9th percentiles, respectively.

$\overline{55 \text { https://optimize.readthedocs.io/en/latest/ }}$ 


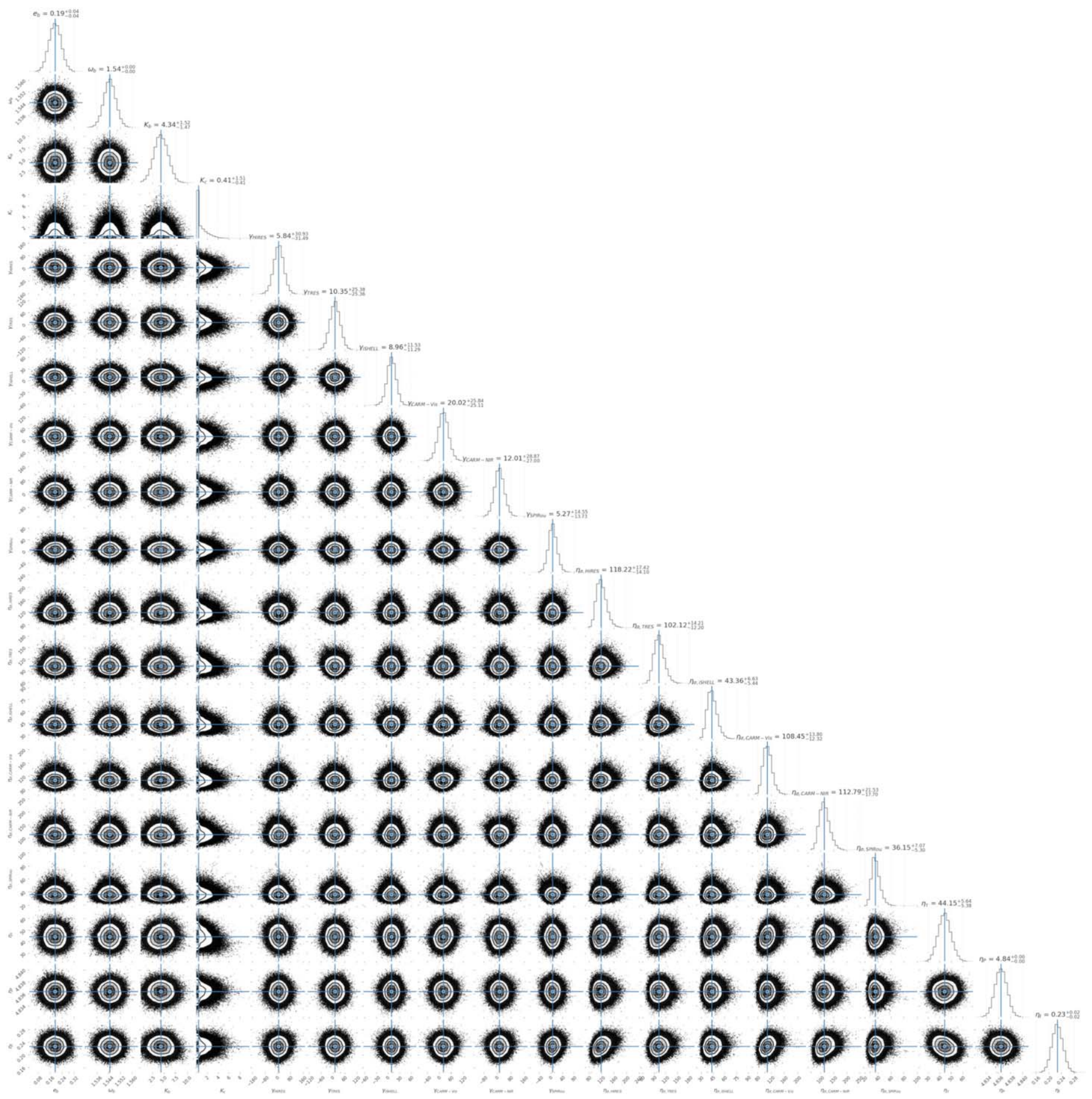

Figure 14. Posterior distributions using disjoint QP kernels (Equation (1)) for each spectrograph to model the stellar activity, including a two-planet model for the transiting planets $\mathrm{b}$ and $\mathrm{c}$. The derived values for $\eta_{\tau}$ and $\eta_{\ell}$ suggest a more dynamic activity model than the $F F^{\prime}$ curve prediction suggests. 


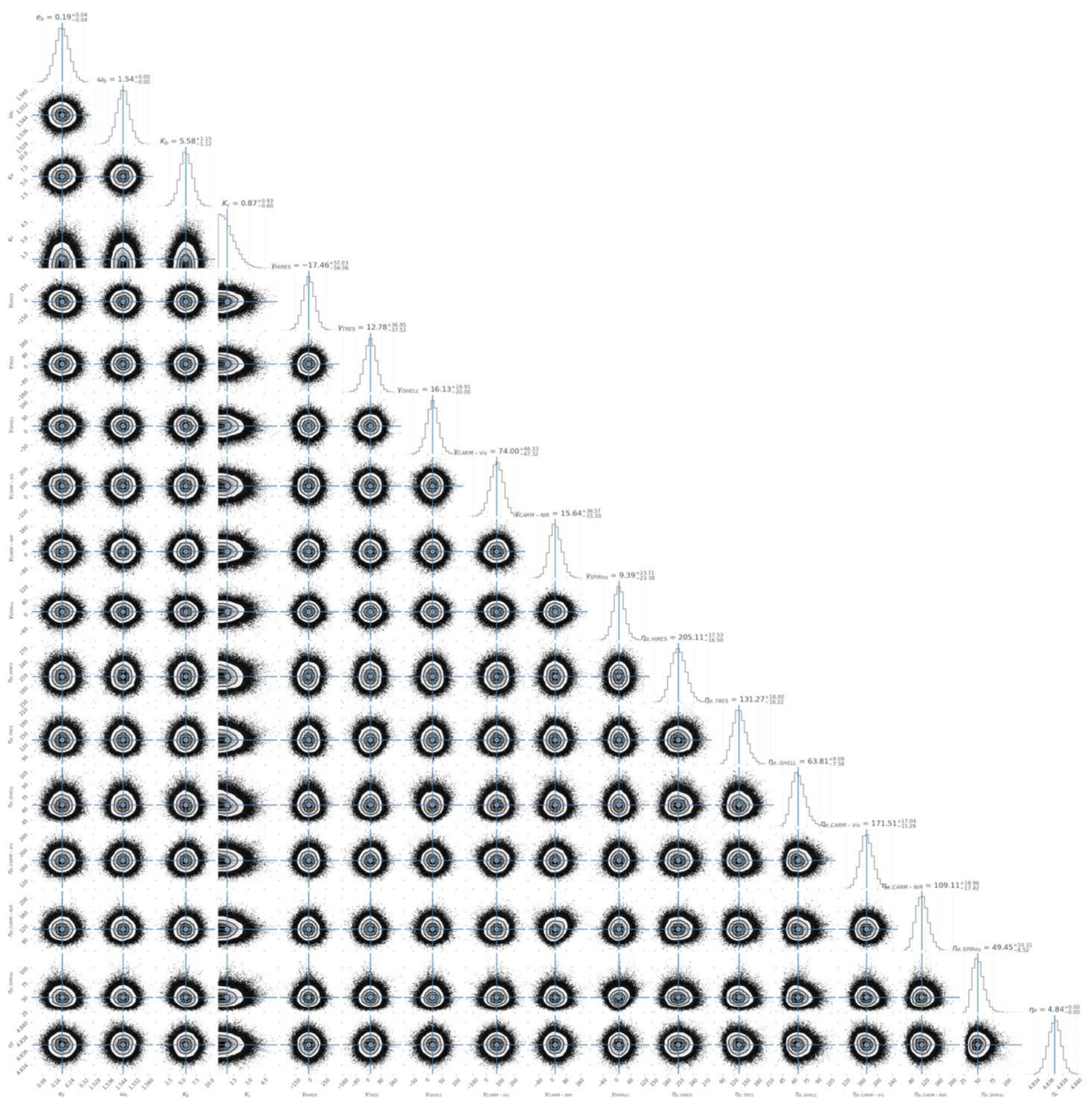

Figure 15. Same as Figure 14, but fixing $\eta_{\tau}=100$ days and $\eta_{\ell}=0.28$. 


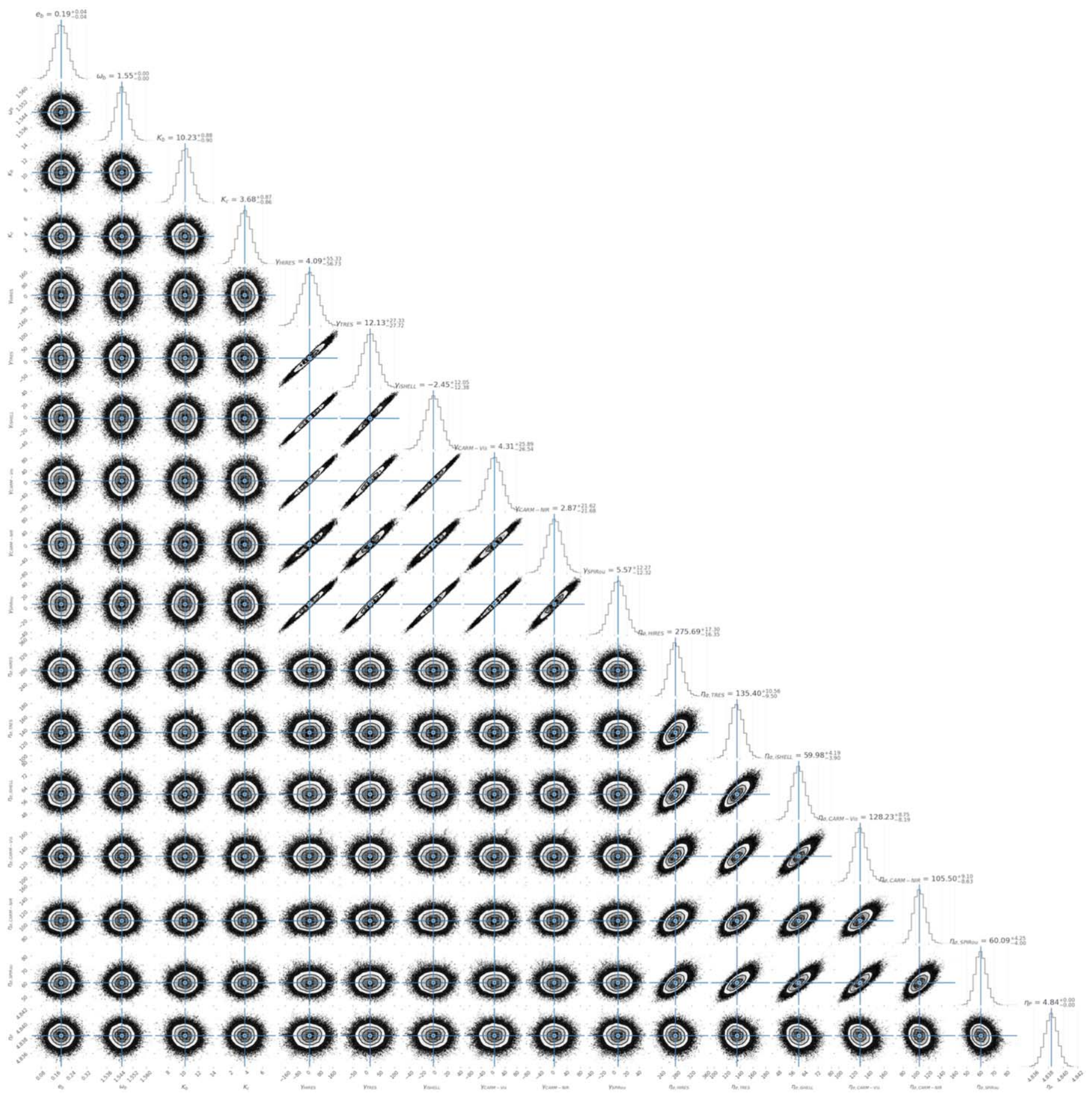

Figure 16. Posterior distributions for a two-planet fit to the RVs using $\boldsymbol{K}_{\boldsymbol{J} 1}$ (Equation (2)) to model the stellar activity. 


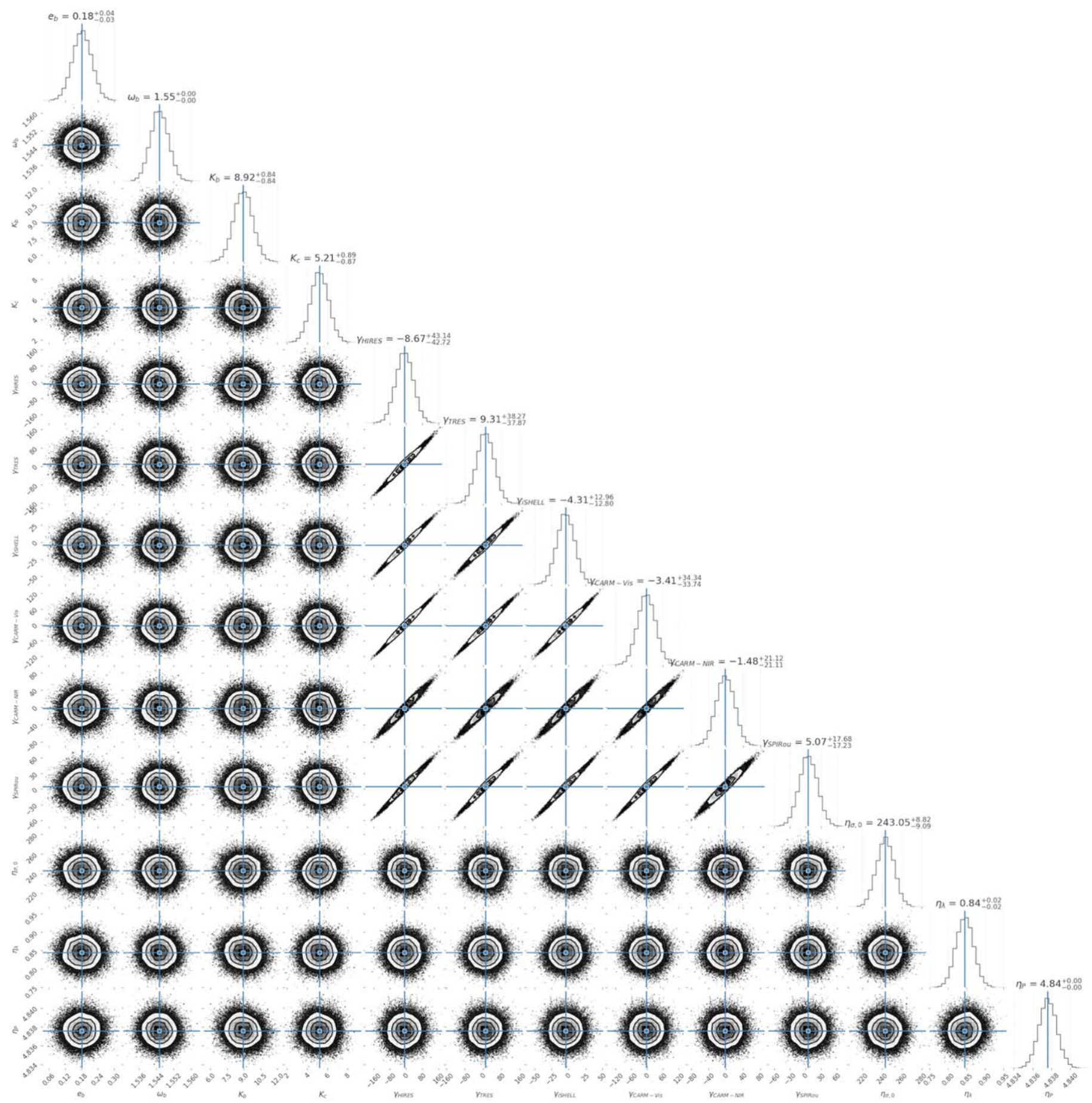

Figure 17. Same as Figure 16, but using $\boldsymbol{K}_{\boldsymbol{J} 2}$ to model the stellar activity. 


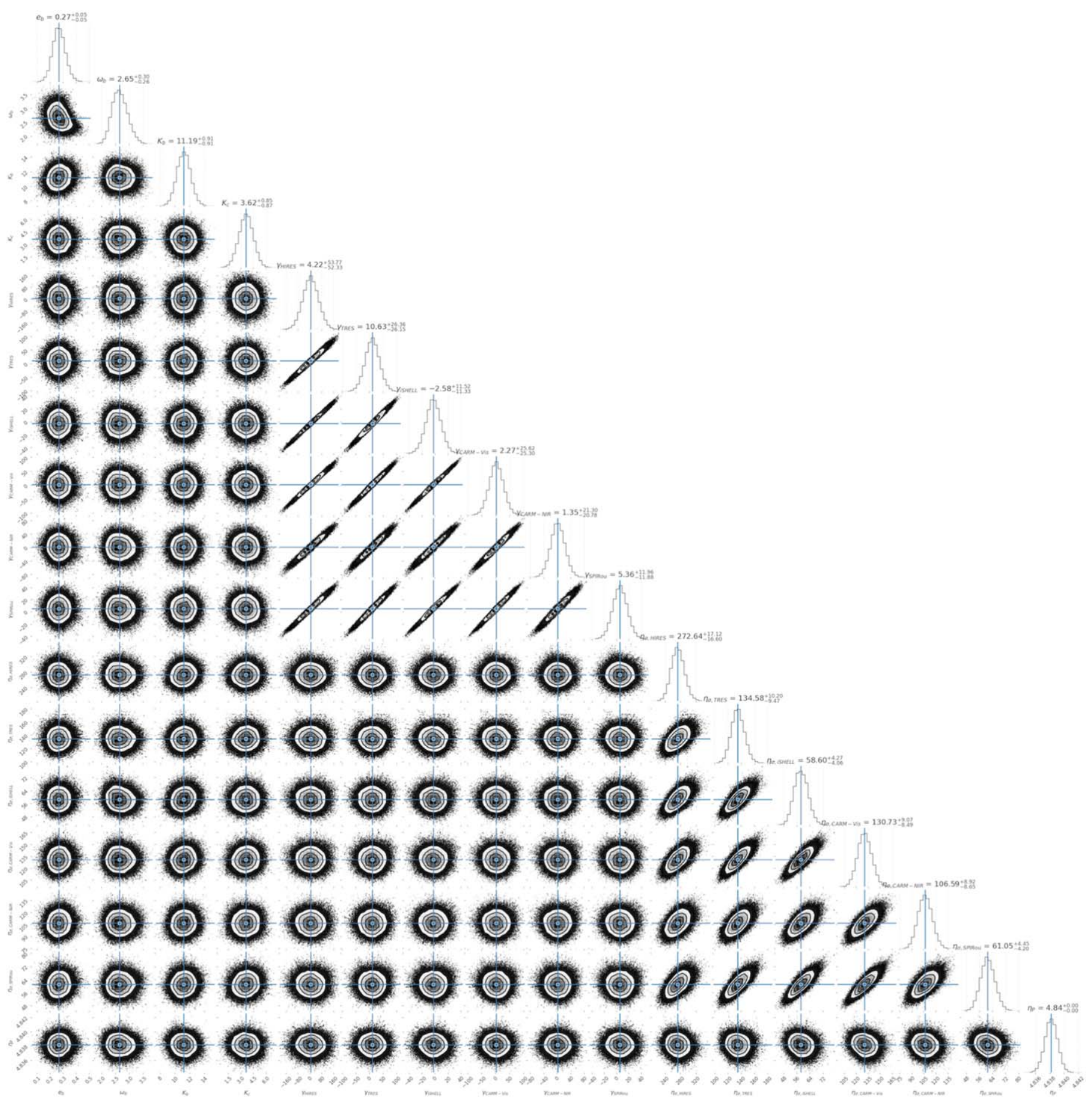

Figure 18. Same as Figure 16, but using less restrictive priors for $e_{b}$ and $\omega_{b}$ as well as $e_{c}$ and $\omega_{c}$. 


\section{Appendix B \\ Optimize}

Optimize ${ }^{56}$ is a generic, high-level optimization package in Python, which generalizes the Bayesian-inspired classes used in RadVel. The primary container (Python class) in optimize is referred to as an "OptProblem;" primary attributes for this object are then helper types to (1) construct the model, (2) compare the data and model with an objective function, and (3) perform the optimization and sample posterior distributions via MCMC methods. Many attributes (such as initial parameters) are shared in multiple layers of this hierarchy for easier access and extension with appropriate methods to propagate changes to each. Optimize does not reimplement specific optimization algorithms, but rather is intended to be a high-level wrapper around such routines (e.g., currently scipy.optimize and emcee).

\section{Appendix C RV Measurements}

Table 7

\begin{tabular}{lcc}
\hline \hline & RV $\left(\mathrm{m} \mathrm{s}^{-1}\right)$ & $\sigma_{\mathrm{RV}}\left(\mathrm{m} \mathrm{s}^{-1}\right)$ \\
\hline Nightly HARPS RVs Analyzed in This Work \\
\hline 2452986.514817 & 73.91 & \\
2453157.898424 & 19.26 & 4.81 \\
2453201.823450 & -32.8 & 1.72 \\
2453468.892370 & -135.2 & 2.56 \\
2453469.843534 & -170.56 & 2.52 \\
2453499.868255 & -43.41 & 2.92 \\
2453521.894368 & -345.97 & 2.52 \\
2453551.803998 & -34.22 & 6.36 \\
2453593.622139 & 137.84 & 2.5 \\
2456568.510365 & -244.53 & 3.33 \\
2456569.500104 & -32.99 & 2.16 \\
2456570.565952 & 193.28 & 1.8 \\
2456772.919271 & -54.34 & 2.17 \\
2456773.918979 & -9.22 & 1.64 \\
2456794.882288 & -119.81 & 0.99 \\
2456795.885873 & 66.36 & 2.53 \\
2456797.857541 & 20.03 & 2.91 \\
2456844.806163 & 162.85 & 3.0 \\
2456982.539577 & -65.03 & 2.18 \\
2457223.648073 & 217.24 & 1.8 \\
2457333.535731 & 94.85 & 2.71 \\
2457493.891905 & 90.65 & 1.29 \\
2457590.712986 & 73.08 & 2.11 \\
2457904.813342 & -287.06 & 1.4 \\
2457917.892902 & 161.13 & 3.36 \\
2458035.528955 & -22.13 & 1.97 \\
2458037.494745 & -41.59 & 1.55 \\
2458206.872213 & -43.23 & 2.39 \\
2458207.892023 & 33.78 & 1.38 \\
2458208.884974 & 82.61 & 1.56 \\
2458591.919473 & 120.82 & 1.42 \\
2458594.904828 & 19.89 & 2.43 \\
2458602.927758 & 9.22 & 1.47 \\
2458605.916360 & -248.46 & 2.33 \\
\hline & -171.02 & \\
\hline 2453182.049556 & & \\
2453195.938633 & Nightly HIRES RVs Analyzed in This Work & \\
& 58.09 & \\
\hline & & \\
\hline & &
\end{tabular}

${ }^{56}$ Documentation: https://optimize.readthedocs.io/en/latest/.
Table 7

(Continued)

\begin{tabular}{|c|c|c|}
\hline $\mathrm{BJD}$ & $\mathrm{RV}\left(\mathrm{m} \mathrm{s}^{-1}\right)$ & $\sigma_{\mathrm{RV}}\left(\mathrm{m} \mathrm{s}^{-1}\right)$ \\
\hline 2453926.030459 & -270.15 & 3.02 \\
\hline 2453926.979641 & 271.86 & 3.18 \\
\hline 2453927.920257 & 68.35 & 2.13 \\
\hline 2453931.079663 & -191.25 & 3.16 \\
\hline 2453932.007322 & 256.77 & 2.76 \\
\hline 2453932.978973 & 7.97 & 2.18 \\
\hline 2453933.944291 & 162.29 & 2.36 \\
\hline 2453934.930418 & -159.79 & 2.73 \\
\hline 2453960.942379 & 257.92 & 2.98 \\
\hline 2453962.030949 & 5.08 & 2.28 \\
\hline 2454636.072465 & -9.82 & 2.53 \\
\hline 2454688.871239 & -133.07 & 2.46 \\
\hline 2454808.692607 & -83.96 & 3.06 \\
\hline 2455015.018198 & -140.72 & 2.86 \\
\hline 2455371.044475 & 100.3 & 1.9 \\
\hline 2455727.981919 & 35.73 & 2.89 \\
\hline 2456638.683238 & 325.16 & 4.18 \\
\hline 2458645.065398 & -77.3 & 7.43 \\
\hline 2459019.062251 & -121.05 & 2.81 \\
\hline 2459025.094631 & -155.66 & 2.48 \\
\hline 2459026.123675 & 399.32 & 3.35 \\
\hline 2459028.098307 & 104.1 & 2.86 \\
\hline 2459029.081905 & 14.31 & 2.53 \\
\hline 2459030.078281 & -131.6 & 2.19 \\
\hline 2459031.106844 & 414.91 & 3.41 \\
\hline 2459032.105577 & -138.95 & 2.71 \\
\hline 2459035.092001 & -76.31 & 2.26 \\
\hline 2459036.101597 & 329.39 & 3.4 \\
\hline 2459040.116030 & 3.72 & 2.12 \\
\hline 2459041.117761 & 153.21 & 3.14 \\
\hline 2459044.942379 & -0.97 & 2.22 \\
\hline 2459051.921900 & 92.94 & 2.44 \\
\hline 2459067.855784 & -21.57 & 3.09 \\
\hline 2459068.895396 & -30.19 & 2.4 \\
\hline 2459071.907618 & 43.81 & 3.37 \\
\hline 2459072.852897 & -8.9 & 2.74 \\
\hline 2459077.821906 & -44.12 & 2.71 \\
\hline 2459078.813958 & -31.15 & 2.45 \\
\hline 2459086.849156 & -147.83 & 3.51 \\
\hline 2459087.868995 & 0.97 & 2.58 \\
\hline 2459088.849805 & 139.3 & 2.57 \\
\hline 2459089.824376 & -38.53 & 3.28 \\
\hline 2459094.815792 & -127.27 & 2.67 \\
\hline 2459097.855698 & -16.26 & 2.6 \\
\hline 2459099.761856 & -139.97 & 3.05 \\
\hline 2459101.904682 & -82.58 & 2.51 \\
\hline 2459114.829534 & -6.09 & 2.08 \\
\hline 2459115.914980 & -92.15 & 2.61 \\
\hline 2459117.857924 & 48.2 & 2.29 \\
\hline 2459121.710044 & 11.92 & 2.38 \\
\hline 2459122.709275 & 58.74 & 2.34 \\
\hline 2459123.703587 & 20.0 & 2.6 \\
\hline 2459151.692854 & 40.18 & 2.41 \\
\hline 2459153.753326 & 95.26 & 2.73 \\
\hline 2459181.680326 & 42.79 & 2.6 \\
\hline 2459187.682871 & 54.26 & 2.47 \\
\hline 2459188.689611 & -6.89 & 2.6 \\
\hline 2459189.684349 & -81.15 & 2.86 \\
\hline \multicolumn{3}{|c|}{ Nightly NIRSPEC RVs Analyzed in This Work } \\
\hline 2453522.56 & 48.5 & 50.0 \\
\hline 2453523.55 & -19.5 & 50.0 \\
\hline 2453596.37 & 111.5 & 50.0 \\
\hline 2453597.38 & 19.5 & 50.0 \\
\hline
\end{tabular}


Table 7

(Continued)

\begin{tabular}{|c|c|c|}
\hline BJD & $\mathrm{RV}\left(\mathrm{m} \mathrm{s}^{-1}\right)$ & $\sigma_{\mathrm{RV}}\left(\mathrm{m} \mathrm{s}^{-1}\right)$ \\
\hline 2453669.19 & 113.5 & 50.0 \\
\hline 2453670.2 & 262.5 & 50.0 \\
\hline 2453928.5 & -74.5 & 50.0 \\
\hline 2453929.45 & -148.5 & 50.0 \\
\hline 2453930.46 & -93.5 & 50.0 \\
\hline 2453931.4 & -24.5 & 50.0 \\
\hline 2454308.43 & 123.5 & 50.0 \\
\hline 2454309.41 & -166.5 & 50.0 \\
\hline 2454311.4 & 20.5 & 50.0 \\
\hline 2454312.36 & -157.5 & 50.0 \\
\hline \multicolumn{3}{|c|}{ Nightly CSHELL RVs Analyzed in This Work } \\
\hline 2455455.85303 & 16.79 & 38.76 \\
\hline 2455479.800206 & 41.04 & 26.96 \\
\hline 2455480.768983 & 143.4 & 56.64 \\
\hline 2455482.756068 & 136.64 & 22.11 \\
\hline 2455523.7002290003 & 0.0 & 20.78 \\
\hline 2455752.0995830004 & 43.59 & 27.48 \\
\hline 2455755.0752310003 & 141.67 & 44.57 \\
\hline 2455758.967657 & -17.29 & 31.52 \\
\hline 2455791.812748 & 123.61 & 54.8 \\
\hline 2455793.8316450003 & 234.74 & 10.87 \\
\hline 2456844.9609169997 & -35.3 & 15.47 \\
\hline 2456917.743247 & -36.65 & 21.07 \\
\hline 2457275.8463990004 & -115.88 & 26.14 \\
\hline 2457551.12512 & -102.38 & 51.72 \\
\hline 2457555.058318 & -150.06 & 20.78 \\
\hline 2457564.0072459998 & -198.34 & 30.4 \\
\hline 2457570.063826 & 82.72 & 36.9 \\
\hline 2457587.023049 & -245.01 & 18.85 \\
\hline 2457598.976608 & -90.04 & 25.01 \\
\hline 2457618.963993 & 8.92 & 19.22 \\
\hline 2457619.947171 & -72.55 & 21.1 \\
\hline
\end{tabular}

\begin{tabular}{lcc}
\multicolumn{2}{c}{ Nightly TRES RVs Analyzed in This Work } & \\
\hline 2456573.68979 & -128.0 & 11.6 \\
2456574.640606 & -45.2 & 11.8 \\
2456575.669816 & 183.9 & 14.4 \\
2456576.660338 & -186.6 & 10.3 \\
2456577.632961 & 203.9 & 12.3 \\
2456578.625675 & -68.1 & 11.9 \\
2456579.634451 & -64.0 & 13.8 \\
2456580.634669 & 173.3 & 13.7 \\
2456581.610641 & -117.8 & 11.3 \\
2456582.623658 & 101.1 & 21.4 \\
2456583.619796 & -35.2 & 9.7 \\
2456584.624069 & -18.7 & 11.0 \\
2456585.596255 & 59.0 & 11.7 \\
2456586.617077 & -22.2 & 12.7 \\
2456587.61581 & 0.0 & 11.7 \\
2456588.591012 & -33.7 & 11.6 \\
2456589.602899 & 45.0 & 11.8 \\
2456590.622533 & -64.4 & 12.1 \\
2456605.581655 & -250.0 & 13.7 \\
2456606.565507 & 225.6 & 8.4 \\
2456607.563214 & -172.0 & 12.1 \\
2456608.58807 & -11.5 & 10.6 \\
2456609.587085 & 211.2 & 13.7 \\
2456610.565465 & -233.4 & 15.0 \\
2456611.571286 & 213.0 & 9.2 \\
2456615.560722 & -193.0 & 13.6 \\
2456616.572477 & 176.9 & 13.8 \\
2456622.557557 & -102.5 & 19.3 \\
2456624.552961 & 85.6 & 46.8 \\
& &
\end{tabular}

Table 7

(Continued)

\begin{tabular}{|c|c|c|}
\hline BJD & $\mathrm{RV}\left(\mathrm{m} \mathrm{s}^{-1}\right)$ & $\sigma_{\mathrm{RV}}\left(\mathrm{m} \mathrm{s}^{-1}\right)$ \\
\hline 2456625.563003 & 38.7 & 24.7 \\
\hline 2458646.96613 & 49.9 & 24.2 \\
\hline 2458647.964611 & 34.3 & 22.0 \\
\hline 2458648.961007 & -44.6 & 17.8 \\
\hline 2458649.958409 & -41.2 & 18.9 \\
\hline 2458650.97301 & 164.3 & 16.2 \\
\hline 2458651.966405 & 116.1 & 17.5 \\
\hline 2458652.981466 & -33.8 & 24.0 \\
\hline 2458657.953559 & 13.6 & 15.7 \\
\hline 2458658.932441 & -107.5 & 17.0 \\
\hline 2458659.912405 & 84.9 & 19.9 \\
\hline 2458665.924079 & 130.6 & 17.8 \\
\hline 2458674.932143 & 198.8 & 14.2 \\
\hline 2458677.882169 & -99.1 & 20.1 \\
\hline 2458685.884899 & 96.1 & 35.5 \\
\hline 2458689.846153 & 110.1 & 14.7 \\
\hline 2458693.842764 & -74.1 & 24.1 \\
\hline 2458730.740018 & 17.1 & 23.4 \\
\hline 2458731.739653 & -35.0 & 23.9 \\
\hline 2458738.70382 & 145.2 & 32.6 \\
\hline 2458742.696035 & -86.4 & 40.1 \\
\hline 2458744.700854 & 234.4 & 25.3 \\
\hline 2458745.730511 & -65.9 & 40.3 \\
\hline 2458758.650170 & 42.1 & 25.3 \\
\hline 2458759.694882 & 114.5 & 28.3 \\
\hline 2458761.645681 & -117.3 & 17.2 \\
\hline 2458762.677499 & 144.6 & 21.8 \\
\hline 2458767.656488 & 107.5 & 33.1 \\
\hline 2458768.653311 & 83.7 & 30.6 \\
\hline 2458769.635106 & 15.1 & 22.5 \\
\hline 2458770.623329 & -112.3 & 18.2 \\
\hline 2458771.612582 & -144.9 & 21.1 \\
\hline 2458772.628816 & 120.2 & 22.4 \\
\hline 2458773.638865 & 91.1 & 25.1 \\
\hline 2458774.591042 & -51.3 & 25.3 \\
\hline 2458775.598544 & -135.9 & 24.1 \\
\hline 2458779.573710 & -86.3 & 24.1 \\
\hline 2458780.566456 & -148.0 & 20.2 \\
\hline 2458782.572973 & 29.7 & 19.0 \\
\hline 2458783.561459 & 123.9 & 30.0 \\
\hline 2458784.56098 & -121.1 & 21.7 \\
\hline 2458786.591881 & -45.4 & 22.6 \\
\hline 2458787.627174 & -2.0 & 29.9 \\
\hline 2458788.570062 & 211.2 & 22.7 \\
\hline
\end{tabular}

Nightly iSHELL RVs Analyzed in This Work

\begin{tabular}{lcc}
\hline 2457684.759584 & 76.97 & 4.94 \\
2457698.745971 & 47.96 & 3.05 \\
2457699.710324 & 47.8 & 14.07 \\
2457850.129559 & 87.59 & 7.24 \\
2457856.130267 & -33.33 & 3.41 \\
2457923.120317 & -1.31 & 4.77 \\
2457931.026094 & -15.53 & 1.59 \\
2457940.000525 & -7.44 & 0.26 \\
2457982.918015 & -2.11 & 11.34 \\
2457983.911491 & -25.53 & 31.67 \\
2457984.906727 & 76.12 & 7.17 \\
2458046.688896 & -53.89 & 6.97 \\
2458047.677872 & 20.45 & 9.09 \\
2458048.684528 & 1.5 & 7.03 \\
2458049.677166 & -68.12 & 11.49 \\
2458660.089282 & 8.42 & 3.31 \\
2458666.92506 & 42.5 & 2.21 \\
2458675.084455 & 71.1 & 4.86
\end{tabular}


Table 7

(Continued)

\begin{tabular}{|c|c|c|}
\hline BJD & $\mathrm{RV}\left(\mathrm{m} \mathrm{s}^{-1}\right)$ & $\sigma_{\mathrm{RV}}\left(\mathrm{m} \mathrm{s}^{-1}\right)$ \\
\hline 2458739.930751 & 85.7 & 4.12 \\
\hline 2458760.71097 & -48.38 & 4.51 \\
\hline 2458761.729422 & -96.54 & 3.37 \\
\hline 2458762.730214 & 16.08 & 6.35 \\
\hline 2458763.779074 & -14.43 & 3.17 \\
\hline 2458764.766543 & 18.96 & 5.06 \\
\hline 2458765.71105 & -39.79 & 7.94 \\
\hline 2458795.700288 & -59.42 & 3.14 \\
\hline 2458796.706964 & -0.86 & 5.37 \\
\hline 2458798.695629 & 32.0 & 4.13 \\
\hline 2458799.69492 & -22.28 & 9.62 \\
\hline 2459069.985765 & 67.29 & 11.02 \\
\hline 2459071.979622 & 24.53 & 3.5 \\
\hline 2459086.915335 & -5.96 & 4.43 \\
\hline 2459087.917716 & 68.09 & 13.17 \\
\hline 2459088.900423 & 39.07 & 5.6 \\
\hline 2459089.900047 & 20.73 & 7.19 \\
\hline 2459090.897927 & 50.16 & 4.41 \\
\hline 2459115.810803 & -7.73 & 5.78 \\
\hline 2459117.805977 & -23.08 & 3.53 \\
\hline 2459118.807183 & -12.72 & 4.15 \\
\hline 2459119.813063 & 3.98 & 5.34 \\
\hline 2459120.804824 & -28.18 & 4.32 \\
\hline 2459122.88224 & 3.77 & 4.62 \\
\hline 2459123.807924 & -16.94 & 5.09 \\
\hline 2459143.789273 & -35.3 & 5.73 \\
\hline 2459145.789894 & 0.86 & 9.38 \\
\hline 2459147.782831 & -7.73 & 4.04 \\
\hline \multicolumn{3}{|c|}{ Nightly IRD RVs Analyzed in This Work } \\
\hline 2458650.116682 & -36.37 & 2.06 \\
\hline 2458653.123778 & 5.82 & 4.63 \\
\hline 2458654.116977 & -62.9 & 1.88 \\
\hline 2458655.126277 & 42.79 & 1.75 \\
\hline 2458679.945743 & 1.82 & 5.4 \\
\hline 2458771.845784 & -1.82 & 3.99 \\
\hline
\end{tabular}

\begin{tabular}{lcc}
\hline \multicolumn{2}{c}{ Nightly CARMENES-NIR RVs Analyzed in This Work } \\
\hline 2458678.567915 & 92.38 & 46.13 \\
2458679.537705 & 315.17 & 54.39 \\
2458680.53478 & 247.69 & 65.59 \\
2458684.568375 & 437.36 & 75.05 \\
2458686.548525 & 275.14 & 49.79 \\
2458687.578395 & 201.29 & 45.37 \\
2458688.584370 & 86.47 & 46.79 \\
2458690.55771 & 180.81 & 23.17 \\
2458691.505425 & 276.31 & 30.81 \\
2458693.54918 & -19.49 & 18.84 \\
2458694.59554 & 164.36 & 19.65 \\
2458695.53909 & -3.88 & 25.06 \\
2458696.523080 & 41.45 & 15.91 \\
2458698.51838 & -112.18 & 13.87 \\
2458699.48421 & 43.11 & 18.82 \\
2458700.47701 & -12.46 & 23.43 \\
2458701.471955 & -38.69 & 30.48 \\
2458702.50203 & -267.32 & 55.65 \\
2458704.48929 & -7.34 & 25.96 \\
2458706.498265 & -60.66 & 24.28 \\
2458711.444815 & -64.13 & 25.69 \\
2458712.45243 & -208.9 & 30.92 \\
2458714.507735 & -1.04 & 29.74 \\
2458715.454715 & 104.33 & 19.43 \\
2458718.45001 & 10.36 & 23.92 \\
2458723.46396 & -10.17 & 29.09
\end{tabular}

Table 7

(Continued)

\begin{tabular}{lcc}
\hline \hline BJD & $\mathrm{RV}\left(\mathrm{m} \mathrm{s}^{-1}\right)$ & $\sigma_{\mathrm{RV}}\left(\mathrm{m} \mathrm{s}^{-1}\right)$ \\
\hline 2458724.419910 & -4.56 & 29.82 \\
2458727.47503 & -162.58 & 33.76 \\
2458742.39457 & -78.27 & 23.94 \\
2458743.368875 & 105.08 & 32.28 \\
2458744.35226 & 96.0 & 17.59 \\
2458745.395085 & -0.64 & 29.71 \\
2458755.39838 & -10.29 & 41.43 \\
2458757.375210 & -54.66 & 41.67 \\
2458759.369515 & 121.73 & 27.43 \\
2458760.30872 & 10.36 & 28.45 \\
2458761.35095 & -120.87 & 25.35 \\
2458763.336130 & 26.53 & 27.49 \\
2458765.32907 & -24.44 & 46.17 \\
2458766.329605 & -141.11 & 33.1 \\
2459049.54651 & 177.28 & 83.51 \\
2459050.5627 & 326.43 & 75.53 \\
2459051.54861 & 164.54 & 58.18 \\
2459059.517 & 128.17 & 38.35 \\
2459060.56397 & 0.64 & 45.11 \\
2459061.51774 & -1.64 & 26.11 \\
2459067.51658 & -22.7 & 35.8 \\
2459070.49213 & -161.95 & 47.51 \\
2459076.47969 & 53.98 & 28.45 \\
2459078.48314 & -53.38 & 47.45 \\
2459079.51194 & 84.71 & 32.93 \\
2459081.44324 & -7.63 & 44.14 \\
2459085.4860900003 & -66.12 & 30.86 \\
2459086.4707 & -5.4 & 35.27 \\
2459087.44822 & 43.18 & 42.15 \\
2459095.462660 & 13.58 & 28.27 \\
2459098.44795 & 15.8 & 34.37 \\
2459099.41347 & 0.86 & 35.8 \\
2459148.30047 & -76.46 & 66.01 \\
2459154.2969 & 25.34 & 61.17 \\
2459161.27302 & -70.52 & 60.54 \\
2459170.25901 & -35.74 & \\
& &
\end{tabular}

\begin{tabular}{lcc}
\hline \multicolumn{1}{c}{ Nightly CARMENES-VIS RVs Analyzed in This Work } \\
\hline 2458678.568125 & -69.66 & 29.45 \\
2458679.537290 & 241.65 & 40.59 \\
2458680.53527 & 79.92 & 15.96 \\
2458684.568335 & 232.51 & 25.58 \\
2458686.54842 & 177.2 & 16.04 \\
2458687.578910 & -17.01 & 20.79 \\
2458688.584365 & -96.42 & 19.21 \\
2458690.5572350 & 114.83 & 11.18 \\
2458691.505425 & 155.65 & 13.93 \\
2458693.549395 & -100.78 & 8.52 \\
2458694.59568 & 135.7 & 6.79 \\
2458695.53932 & 39.93 & 10.69 \\
2458696.522970 & 57.79 & 6.7 \\
2458698.518795 & -108.57 & 4.86 \\
2458699.484225 & 81.68 & 8.55 \\
2458700.47709 & 10.01 & 6.31 \\
2458701.472325 & -1.34 & 7.9 \\
2458702.502 & -163.13 & 16.45 \\
2458704.489635 & 79.4 & 7.75 \\
2458706.498955 & -53.41 & 13.14 \\
2458711.445175 & -68.26 & 9.3 \\
2458712.452575 & -145.53 & 8.27 \\
2458714.507445 & 74.89 & 11.13 \\
2458715.454290 & 119.79 & 6.96 \\
2458718.449865 & -8.15 & 7.88 \\
2458723.46386 & 10.82 & 9.14 \\
& &
\end{tabular}


Table 7

(Continued)

\begin{tabular}{|c|c|c|}
\hline BJD & $\mathrm{RV}\left(\mathrm{m} \mathrm{s}^{-1}\right)$ & $\sigma_{\mathrm{RV}}\left(\mathrm{m} \mathrm{s}^{-1}\right)$ \\
\hline 2458724.42014 & 32.17 & 9.24 \\
\hline 2458727.47467 & -120.64 & 15.58 \\
\hline 2458742.394525 & -111.51 & 6.47 \\
\hline 2458743.36923 & 109.79 & 10.05 \\
\hline 2458744.35274 & 44.66 & 6.63 \\
\hline 2458745.394785 & -8.64 & 10.77 \\
\hline 2458755.399070 & -52.31 & 12.96 \\
\hline 2458757.37524 & -40.49 & 11.8 \\
\hline 2458759.369535 & 173.63 & 10.1 \\
\hline 2458760.30896 & -50.26 & 9.67 \\
\hline 2458761.350815 & -127.71 & 8.52 \\
\hline 2458763.33635 & 21.39 & 6.78 \\
\hline 2458765.32879 & -83.71 & 9.85 \\
\hline 2458766.32998 & -150.2 & 7.98 \\
\hline 2459049.54617 & -9.06 & 23.94 \\
\hline 2459050.56301 & 261.02 & 21.89 \\
\hline 2459051.54856 & -21.26 & 27.7 \\
\hline 2459059.51739 & 21.86 & 12.02 \\
\hline 2459060.56385 & 56.21 & 15.25 \\
\hline 2459061.51767 & 3.43 & 9.96 \\
\hline 2459067.51635 & -61.79 & 14.71 \\
\hline 2459070.49224 & -120.99 & 17.32 \\
\hline 2459076.47973 & 74.87 & 9.62 \\
\hline 2459078.48285 & -66.09 & 12.86 \\
\hline 2459079.51202 & 159.74 & 17.82 \\
\hline 2459081.4433 & 18.72 & 16.94 \\
\hline 2459085.48611 & -103.96 & 11.19 \\
\hline 2459086.47053 & 0.0 & 11.41 \\
\hline 2459087.44817 & -22.76 & 10.14 \\
\hline 2459095.46273 & -17.99 & 15.28 \\
\hline 2459098.44771 & 36.94 & 14.87 \\
\hline 2459099.41364 & 1.26 & 13.44 \\
\hline 2459113.41579 & 77.58 & 40.76 \\
\hline 2459148.3007 & -199.06 & 41.82 \\
\hline 2459154.29727 & 80.14 & 23.03 \\
\hline 2459161.27325 & -25.96 & 17.31 \\
\hline 2459170.25874 & -52.59 & 15.02 \\
\hline
\end{tabular}

Nightly MINERVA-Australis RVs Analyzed in This Work

\begin{tabular}{ccc}
\hline 2458683.150648 & 89.06 & 38.22 \\
2458684.165833 & 208.9 & 10.54 \\
2458711.967436 & -67.42 & 12.5 \\
2458716.998287 & -61.47 & 10.95 \\
2458719.003218 & 175.13 & 22.44 \\
2458720.062263 & 81.08 & 6.24 \\
2458725.959649 & 32.34 & 104.73 \\
2458738.040897 & -55.32 & 7.36 \\
2458739.963764 & 235.89 & 6.39
\end{tabular}

Table 7

(Continued)

\begin{tabular}{lcc}
\hline \hline BJD & $\mathrm{RV}\left(\mathrm{m} \mathrm{s}^{-1}\right)$ & $\sigma_{\mathrm{RV}}\left(\mathrm{m} \mathrm{s}^{-1}\right)$ \\
\hline 2458740.989093 & -125.63 & 5.43 \\
2458741.999722 & -128.95 & 8.06 \\
2458743.006845 & 0.0 & 5.15 \\
2458792.965243 & -13.18 & 9.52
\end{tabular}

\begin{tabular}{lcc}
\hline \multicolumn{3}{c}{ Nightly CHIRON RVs } \\
\hline 2458740.72096 & 1.48 & 45.24 \\
2458741.713716 & 149.09 & 46.54 \\
2458742.711554 & -54.11 & 37.64 \\
2458762.643643 & 112.47 & 56.45 \\
2458763.64337 & -44.57 & 36.34 \\
2458764.629147 & 72.97 & 42.89 \\
2458765.631185 & -310.79 & 58.25 \\
2458766.614416 & -169.88 & 44.79 \\
2458795.574927 & 51.27 & 68.8 \\
2458796.570968 & -1.48 & 71.87 \\
2458797.56992 & -11.17 & 37.07 \\
2458798.597397 & 359.32 & 52.94 \\
\hline
\end{tabular}

2458798.597397

Nightly SPIRou RVs Analyzed in This Work

$2458744.8212 \quad 59.5 \quad 5.0$

$2458750.7542 \quad-18.2 \quad 5.0$

$2458751.7453 \quad-52.4 \quad 5.0$

$2458752.7898 \quad 27.9 \quad 5.0$

$2458758.7288 \quad 51.3 \quad 5.0$

$2458759.8053 \quad 69.2 \quad 5.0$

$2458760.7278 \quad-29.6 \quad 5.0$

$2458761.7305-187.2 \quad 5.0$

$2458762.7315 \quad-2.9 \quad 5.0$

$2458764.7571 \quad 34.5 \quad 5.0$

$2458765.7694-39.8 \quad 5.0$

$2458769.7438 \quad 22.1 \quad 5.0$

$2458770.7407 \quad-41.6 \quad 5.0$

2458771.7212
2458772.7416

$\begin{array}{lll}2458772.7416 & 19.4 & 5.0\end{array}$

$\begin{array}{lll}2458787.7155 & 28.3 & 5.0\end{array}$

$2458788.7045 \quad 41.3 \quad 5.0$

$2458789.7367 \quad-32.6 \quad 5.0$

$\begin{array}{lll}2458790.701 & -90.1 & 5.0\end{array}$

$2458791.6983 \quad 2.1 \quad 5.0$

$2458792.6976 \quad 19.1 \quad 5.0$

$\begin{array}{lll}2458796.6859 & -13.5 & 5.0\end{array}$

$\begin{array}{lll}2458797.7098 & 19.7 & 5.0\end{array}$

$2458798.6873 \quad 27.0 \quad 5.0$

$\begin{array}{lll}2458799.6883 & -10.3 & 5.0\end{array}$

$2458800.6896 \quad-46.2 \quad 5.0$

$\begin{array}{lll}2458801.6873 & 0.0 & 5.0\end{array}$




\section{Appendix D}

Fitting the Full RV Data Set

Here, we present fits to the full radial-velocity data set (see Section 2). Although the baseline of the full data set is nearly 17 yr (first epoch in 2003 December), the uncertainties for the period and time of transit for AU Mic b and $\mathrm{c}$ are small enough to be fixed (see Table 2). We only use kernel $\boldsymbol{K}_{\boldsymbol{J} 2}$ (Equation (3)) to model the stellar activity, as we do not seek to fit for per-spectrograph activity amplitudes for data sets with $\lesssim 10$ measurements. A first-order estimation for the secular acceleration (Choi et al. 2013) of AU Mic is negligible,

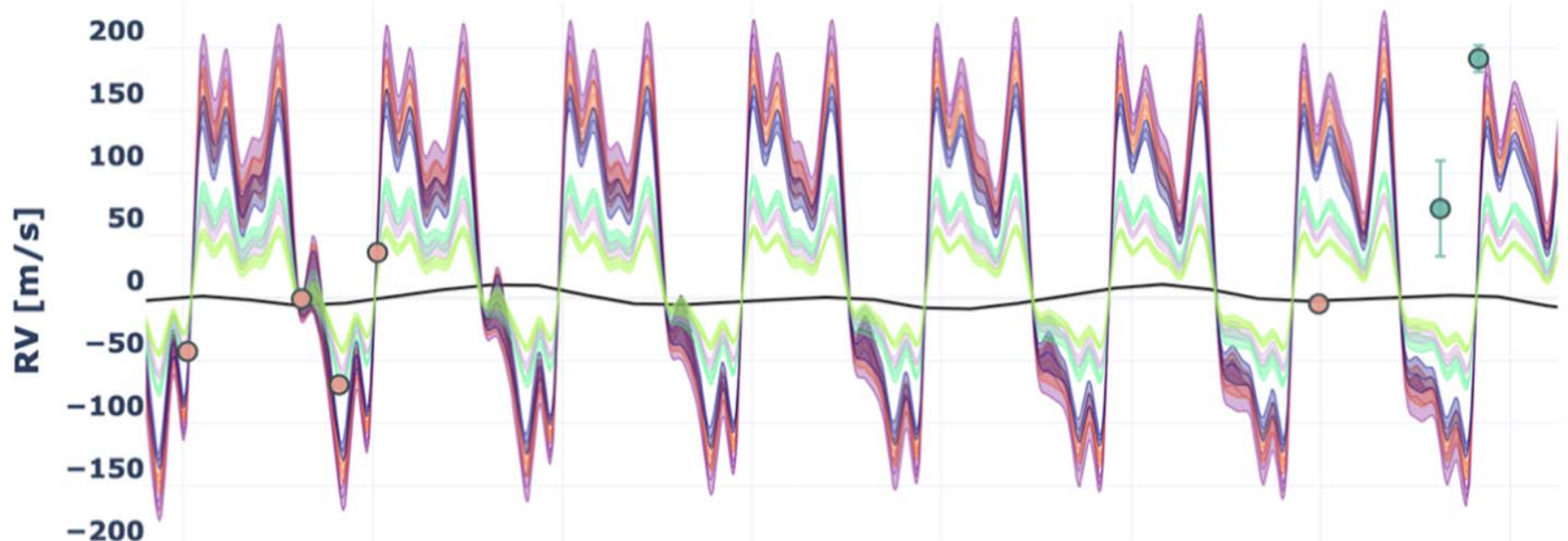

$-200$

8650

8655

8660

8665

8670

8675

8680

8685

250
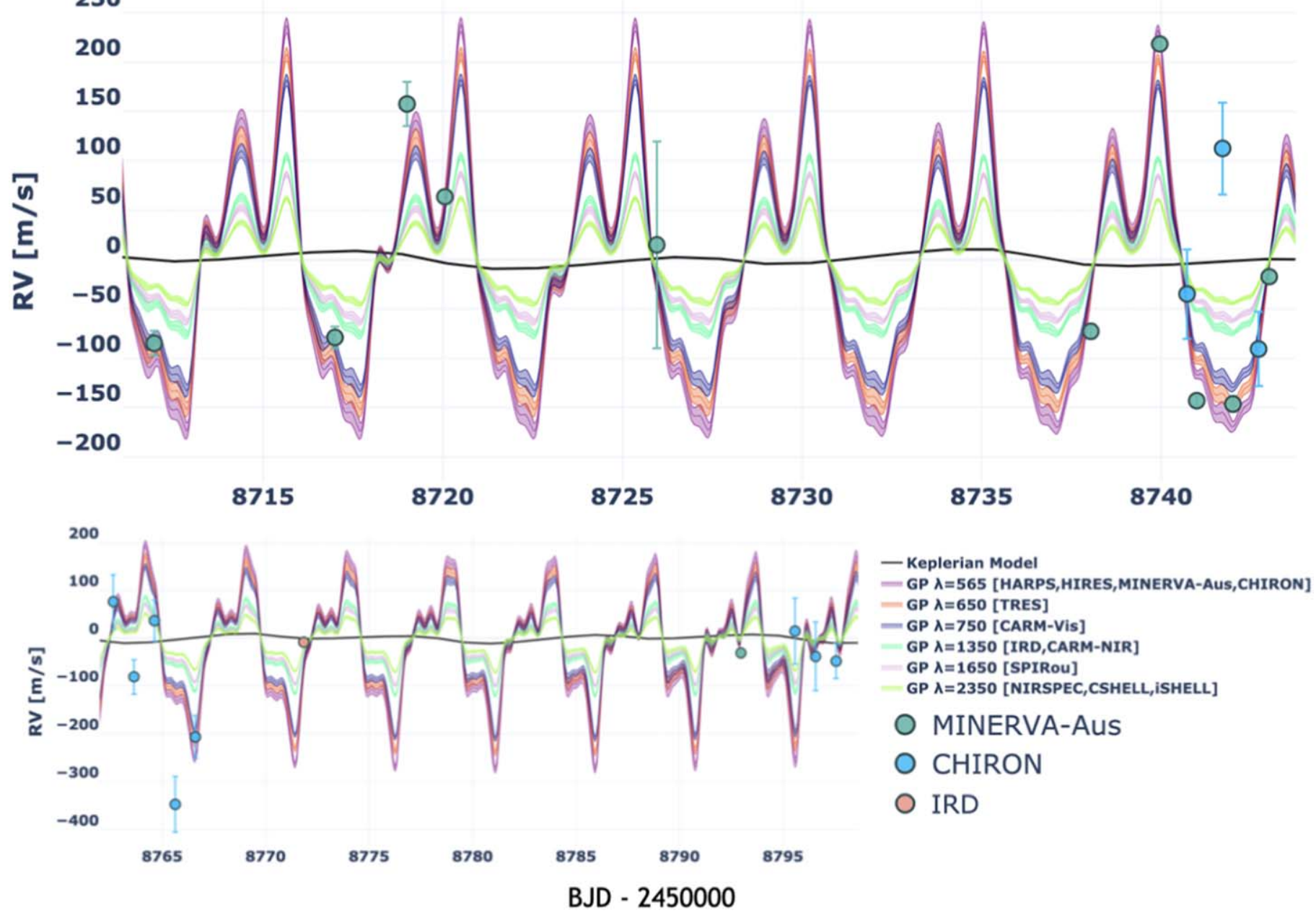

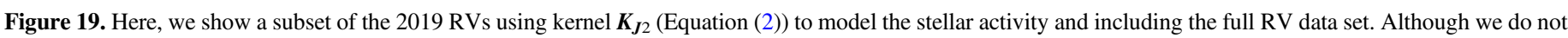

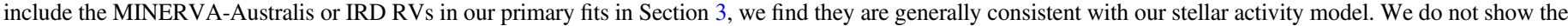
phased CHIRON RVs, due to their larger residuals. 

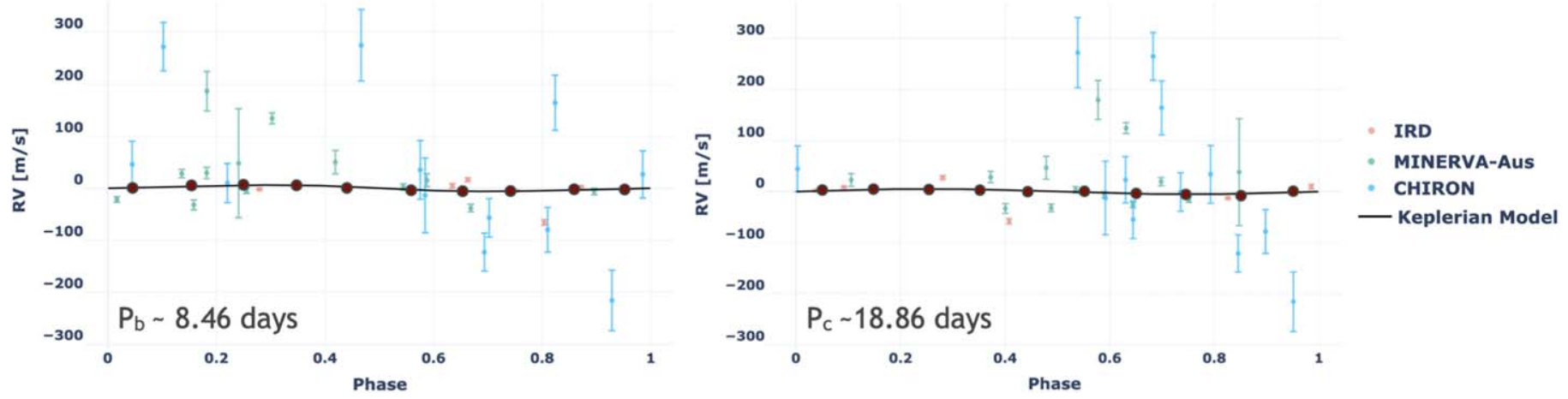

Figure 20. Same as Figure 19, but showing the phased RVs. 


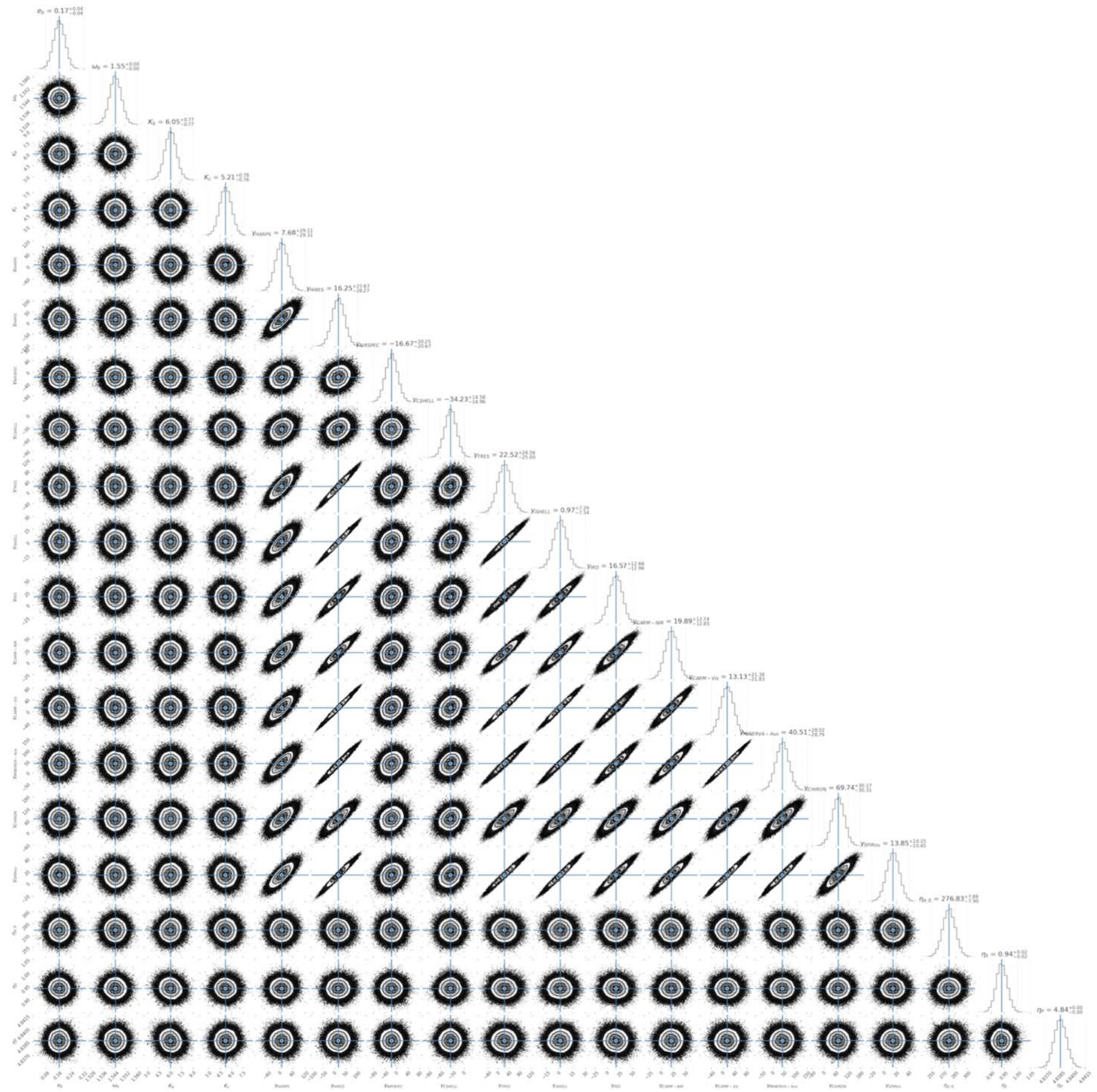

Figure 21. Posterior distributions for a two-planet fit to the full RV data set using $\boldsymbol{K}_{\boldsymbol{J} 2}$ to model the stellar activity. Blue lines correspond to the 50th percentile of the distribution. Upper and lower uncertainties correspond to the 15.9 th and 84.1 st percentiles, respectively. The semi-amplitude $K_{b}$ is $\approx 30 \%$ smaller than the subset of 2019-2020 data yields (Table 3), but $K_{c}$ is relatively unchanged.

given the precision of our measurements and baseline $\left(\Delta \mathrm{RV}<3 \mathrm{~cm} \mathrm{~s}^{-1}\right)$, so no long-term linear or quadratic trend is used. The GPs and Keplerian model are shown in Figure 19, and the phased RVs are shown in Figure 20. The posteriors are shown in Figure 21.

\section{ORCID iDs}

Bryson L. Cale (1) https://orcid.org/0000-0001-6279-0595 Peter Plavchan (ํ) https://orcid.org/0000-0002-8864-1667 Eric Gaidos (1) https://orcid.org/0000-0002-5258-6846 Jonathan Gagné (i) https://orcid.org/0000-0002-2592-9612
Peter Gao (ํ) https://orcid.org/0000-0002-8518-9601

Stephen R. Kane (1) https://orcid.org/0000-0002-7084-0529

Víctor J. S. Béjar (i) https://orcid.org/0000-0002-5086-4232

Nicolas Lodieu (1) https://orcid.org/0000-0002-3612-8968

Ignasi Ribas (1) https://orcid.org/0000-0002-6689-0312

Enric Pallé (ib https://orcid.org/0000-0003-0987-1593

Andrew W. Howard (ㄱ) https://orcid.org/0000-0001-8638-0320

Benjamin J. Fulton (1) https://orcid.org/0000-0003-3504-5316

Kevin I. Collins (1) https://orcid.org/0000-0003-2781-3207

Justin Wittrock iㅣ https://orcid.org/0000-0002-7424-9891

Emily A. Gilbert (1) https://orcid.org/0000-0002-0388-8004 
Thomas Barclay (1) https://orcid.org/0000-0001-7139-2724

Robert Wittenmyer (i) https://orcid.org/0000-0001-9957-9304

Duncan Wright (i) https://orcid.org/0000-0001-7294-5386

Brett Addison (1) https://orcid.org/0000-0003-3216-0626

Teruyuki Hirano (1) https://orcid.org/0000-0003-3618-7535

Motohide Tamura (1) https://orcid.org/0000-0002-6510-0681

Norio Narita (1) https://orcid.org/0000-0001-8511-2981

Samuel N. Quinn (1) https://orcid.org/0000-0002-8964-8377

David W. Latham (1) https://orcid.org/0000-0001-9911-7388

Gilbert A. Esquerdo (ib https://orcid.org/0000-0002-9789-5474

Michael L. Calkins 나 https://orcid.org/0000-0002-2830-5661

Jon Jenkins (1) https://orcid.org/0000-0002-4715-9460

Joseph D. Twicken (1) https://orcid.org/0000-0002-6778-7552

Christopher Burke 지 https://orcid.org/0000-0002-7754-9486

Patricia Boyd (i) https://orcid.org/0000-0003-0442-4284

\section{References}

Addison, B., Wright, D. J., Wittenmyer, R. A., et al. 2019, PASP, 131, 115003

Addison, B. C., Wright, D. J., Nicholson, B. A., et al. 2021, MNRAS, 502,3704

Aigrain, S., Pont, F., \& Zucker, S. 2012, MNRAS, 419, 3147

Akaike, H. 1974, ITAC, 19, 716

Allard, F., Homeier, D., \& Freytag, B. 2012, RSPTA, 370, 2765

Anglada-Escudé, G., \& Butler, R. P. 2012, ApJS, 200, 15

Bailey, J. I., White, R. J., Blake, C. H., et al. 2012, ApJ, 749, 16

Barnes, S. I., Gibson, S., Nield, K., \& Cochrane, D. 2012, Proc. SPIE, 8446, 844688

Batalha, N. E., Lewis, T., Fortney, J. J., et al. 2019, ApJL, 885, L25

Bauer, F. F., Zechmeister, M., Kaminski, A., et al. 2020, A\&A, 640, A50

Buchhave, L. A., Bakos, G. Á., Hartman, J. D., et al. 2010, ApJ, 720, 1118

Burnham, K. P., \& Anderson, D. R. 2002, Model Selection and Multimodel Inference (Berlin: Springer)

Caballero, J. A., Guàrdia, J., López del Fresno, M., et al. 2016, Proc. SPIE, 9910, 99100E

Cale, B., Plavchan, P., LeBrun, D., et al. 2019, AJ, 158, 170

Chen, J., \& Kipping, D. 2017, ApJ, 834, 17

Choi, J., McCarthy, C., Marcy, G. W., et al. 2013, ApJ, 764, 131

Cretignier, M., Dumusque, X., Allart, R., Pepe, F., \& Lovis, C. 2020, A\&A, 633, A76

Czesla, S., Schröter, S., Schneider, C. P., et al. 2019, PyA: Python Astronomyrelated Packages, Astrophysics Source Code Library, ascl:1906.010

Desort, M., Lagrange, A. M., Galland, F., Udry, S., \& Mayor, M. 2007, A\&A, 473, 983

Donati, J.-F., Kouach, D., Lacombe, M., et al. 2018, in SPIRou: A NIR Spectropolarimeter/High-Precision Velocimeter for the CFHT, ed. H. J. Deeg \& J. A. Belmonte (Berlin: Springer), 107

Dumusque, X. 2018, A\&A, 620, A47

Dumusque, X., Boisse, I., \& Santos, N. C. 2014, ApJ, 796, 132

Foreman-Mackey, D., Hogg, D. W., Lang, D., \& Goodman, J. 2013, PASP, 125,306

Foreman-Mackey, D., Price-Whelan, A., Vousden, W., et al. 2020, dfm/corner.py: corner.py v2.1.0.rc1, v2.1.0.rc1, Zenodo, doi:10.5281/zenodo.3937526

Fulton, B. J., Petigura, E. A., Blunt, S., \& Sinukoff, E. 2018, PASP, 130 , 044504

Fúrész, G. 2008, PhD thesis, University of Szeged, Hungary, http://www.sao. arizona.edu/html/FLWO/60/TRES/GABORthesis.pdf

Gaia Collaboration, Brown, A. G. A., Vallenari, A., et al. 2018, A\&A, 616, A1

Gagné, J., Plavchan, P., Gao, P., et al. 2016, ApJ, 822, 40

Gao, P., Plavchan, P., Gagné, J., et al. 2016, PASP, 128, 104501

Gilbert, E. A., Barclay, T., \& Quintana, E. V. 2021, arXiv:2109.03924

Greene, T. P., Tokunaga, A. T., Toomey, D. W., \& Carr, J. B. 1993, Proc. SPIE, 1946, 313

Grunblatt, S. K., Howard, A. W., \& Haywood, R. D. 2015, ApJ, 808, 127
Harris, C. R., Millman, K. J., van der Walt, S. J., et al. 2020, Natur, 585, 357 Haywood, R. D. 2015, PhD thesis, University of St. Andrews

Haywood, R. D., Collier Cameron, A., Queloz, D., et al. 2014, MNRAS, 443, 2517

Hirano, T., Krishnamurthy, V., Gaidos, E., et al. 2020a, ApJL, 899, L13

Hirano, T., Kuzuhara, M., Kotani, T., et al. 2020b, PASJ, 72, 93

Howard, A. W., Johnson, J. A., Marcy, G. W., et al. 2010, ApJ, 721, 1467

Hunter, J. D. 2007, CSE, 9,90

Inc., P. T. 2015, Collaborative Data Science (Montreal, QC: Plotly Technologies Inc.), https://plot.ly

Jeffreys, H. 1946, RSPSA, 186, 453

Jenkins, J. M., Twicken, J. D., McCauliff, S., et al. 2016, Proc. SPIE, 9913, 99133E

Jones, E., Oliphant, T., Peterson, P., et al. 2001, SciPy: Open Source Scientific Tools for Python, http://www.scipy.org/

Klein, B., Donati, J.-F., Moutou, C., et al. 2021, MNRAS, 502, 188

Kotani, T., Tamura, M., Nishikawa, J., et al. 2018, Proc. SPIE, 10702, 1070211

Lam, S. K., Pitrou, A., \& Seibert, S. 2015, Numba: A llvm-based Python jit Compiler

Lo Curto, G., Beniflah, T., Burrows, A., et al. 2010, Msngr, 142, 42

López-Morales, M., Haywood, R. D., Coughlin, J. L., et al. 2016, AJ, 152, 204

Mamajek, E. E., \& Bell, C. P. M. 2014, MNRAS, 445, 2169

Martioli, E., Hébrard, G., Correia, A. C. M., Laskar, J., \& Lecavelier des Etangs, A. 2021, A\&A, 649, A177

Mayor, M., Pepe, F., Queloz, D., et al. 2003, Msngr, 114, 20

McLean, I. S., Becklin, E. E., Bendiksen, O., et al. 1998, Proc. SPIE, 3354, 566 Meunier, N., \& Lagrange, A. M. 2013, A\&A, 551, A101

NASA Exoplanet Archive 2019, Confirmed Planets Table, IPAC, doi:10. 26133/NEA1

Pecaut, M. J., \& Mamajek, E. E. 2013, ApJS, 208, 9

Piskunov, N. E., \& Valenti, J. A. 2002, A\&A, 385, 1095

Plavchan, P., Barclay, T., Gagné, J., et al. 2020, Natur, 582, 497

Quinn, S. N., White, R. J., Latham, D. W., et al. 2014, ApJ, 787, 27

Quirrenbach, A., Amado, P. J., Ribas, I., et al. 2018, Proc. SPIE, 10702, 107020W

Rajpaul, V., Aigrain, S., Osborne, M. A., Reece, S., \& Roberts, S. 2015, MNRAS, 452, 2269

Rasmussen, C. E., \& Williams, C. K. I. 2006, Gaussian Processes for Machine Learning (Berlin: Springer)

Rayner, J., Tokunaga, A., Jaffe, D., et al. 2016, Proc. SPIE, 9908, 990884

Reiners, A., Bean, J. L., Huber, K. F., et al. 2010, ApJ, 710, 432

Reiners, A., \& Zechmeister, M. 2020, ApJS, 247, 11

Ricker, G. R., Winn, J. N., Vanderspek, R., et al. 2015, JATIS, 1, 014003

Robertson, P., Stefansson, G., Mahadevan, S., et al. 2020, ApJ, 897, 125

Smith, J. C., Stumpe, M. C., Van Cleve, J. E., et al. 2012, PASP, 124, 1000

Stumpe, M. C., Smith, J. C., Catanzarite, J. H., et al. 2014, PASP, 126, 100

Stumpe, M. C., Smith, J. C., Van Cleve, J. E., et al. 2012, PASP, 124, 985

Swade, D., Fleming, S., Jenkins, J. M., et al. 2018, Proc. SPIE, 10704, 1070415

Szabó, G. M., Gandolfi, D., Brandeker, A., et al. 2021, A\&A, 654, A159

Tody, D. 1993, in ASP Conf. Ser. 52, Astronomical Data Analysis Software and Systems II, ed. R. J. Hanisch, R. J. V. Brissenden, \& J. Barnes (San Francisco, CA: ASP), 173

Tokovinin, A., Fischer, D. A., Bonati, M., et al. 2013, PASP, 125, 1336

Toledo-Padrón, B., Suárez Mascareño, A., González Hernández, J. I., et al. 2021, A\&A, 648, A20

Trifonov, T., Kürster, M., Zechmeister, M., et al. 2018, A\&A, 609, A117

Tronsgaard, R., Buchhave, L. A., Wright, J. T., Eastman, J. D., \& Blackman, R. T. 2019, MNRAS, 489, 2395

Virtanen, P., Gommers, R., Oliphant, T. E., et al. 2020, NatMe, 17, 261

Vogt, S. S., Allen, S. L., Bigelow, B. C., et al. 1994, Proc. SPIE, 2198, 362

Wise, A. W., Dodson-Robinson, S. E., Bevenour, K., \& Provini, A. 2018, AJ, 156,180

Wittenmyer, R. A., Horner, J., Carter, B. D., et al. 2018, arXiv:1806.09282

Zechmeister, M., \& Kürster, M. 2018, GLS: Generalized Lomb-Scargle Periodogram, Astrophysics Source Code Library, ascl:1807.019

Zechmeister, M., Reiners, A., Amado, P. J., et al. 2018, A\&A, 609, A12 